University of Redlands

\title{
Hopi Horizon Analysis with GIS
}

\author{
A Major Individual Project submitted in partial satisfaction of the requirements \\ for the degree of Master of Science in Geographic Information Systems \\ by \\ Alicia Nicole Barnash \\ Mark Kumler, Ph.D., Committee Chair \\ Wesley Bernardini, Ph.D.
}

March 2011 
Hopi Horizon Analysis

Copyright (C) 2011

by

Alicia Nicole Barnash 
The report of Alicia Nicole Barnash is approved.
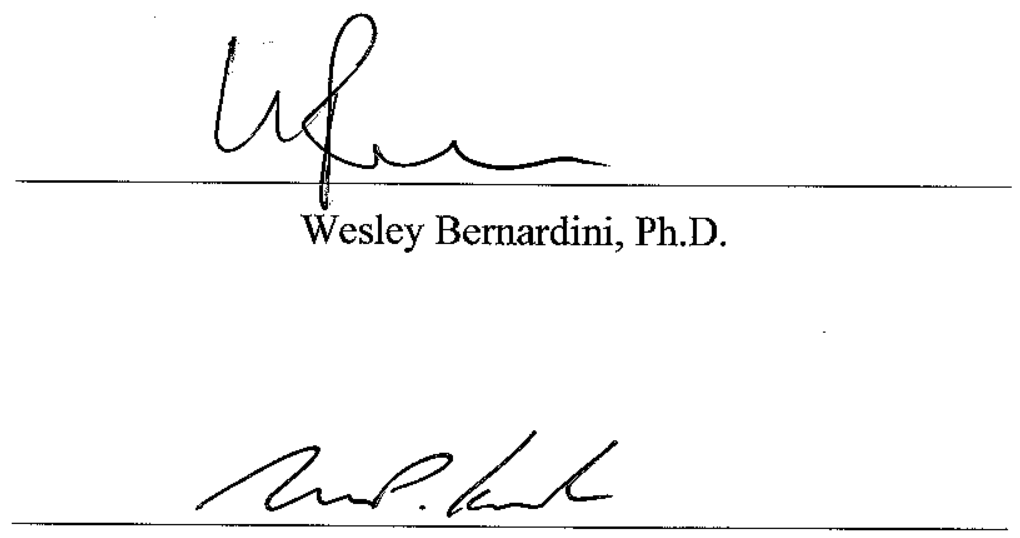

Mark Kumler, Ph.D., Committee Chair

March 2011 



\section{Acknowledgements}

I would like to begin by thanking my committee chair and advisor, Dr. Mark Kumler. He contributed many hours of his time to meeting with me, exploring creative ways to solve problems, and helping me work through challenging aspects of the project. His constant encouragement and enthusiasm was invaluable in helping me navigate through the University of Redlands MS GIS program from start to finish.

I would also like to express gratitude to my project client, Dr. Wesley Bernardini. It has been a pleasure working with him for the past 1.16 years. He was always ready to help with any questions I had, was patient and understanding when tasks took me much longer to complete than originally estimated. I look forward to continuing work with him in the future on this exciting subject matter.

Finally, I would like to acknowledge my parents, Vicki and Mike Barnash for providing all fundamental necessities for living. They have supported me in the support of my academic goals not only for the past year while I studied GIS at Redlands, but also when I was a little person at Idyllwild Elementary, and again when I was a teenager at Hemet High, and yet again when I was studying Physics as an undergrad at the University of Redlands. All of their support over those long years made it possible for me to get to this stage in my life. 



\begin{abstract}
Hopi Horizon Analysis with GIS

by

Alicia Nicole Barnash
\end{abstract}

This paper is a report on the planning, design, and implementation of the Hopi Horizon Analysis project. The project client is Dr. Wesley Bernardini, a professor of Sociology and Anthropology at the University of Redlands. Dr. Bernardini wanted to know in what ways the visual properties of horizons influenced how the Hopi culture retained knowledge of distant places as they migrated to new locations. This project developed a method for ranking the visual significance of mountain peaks along an observer's visual horizon using the Douglas-Peucker line simplification algorithm. The project implementation involved the design and construction of a geodatabase, the development of custom geoprocessing tools, and the implementation of a technique for visualizing the changes in peak significance over time. 



\section{Table of Contents}

Chapter 1 - Introduction ............................................................................................... 1

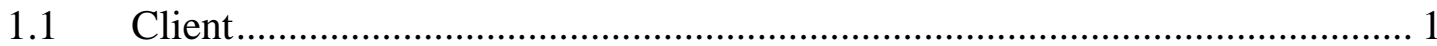

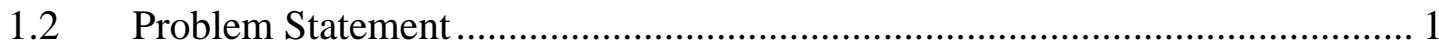

$1.3 \quad$ Proposed Solution ............................................................................... 1

1.3.1 Goals and Objectives ........................................................................... 2

1.3.2 Scope

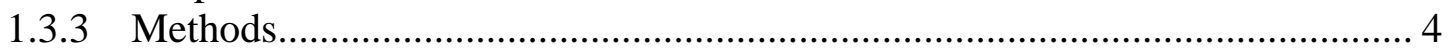

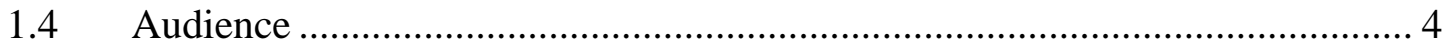

1.5 Overview of the Rest of this Report ........................................................ 5

Chapter 2 - Background and Literature Review ............................................................. 7

$2.1 \quad$ Visual Properties of Landscapes ............................................................. 7

2.2 Quantifying Prominence of Peaks ................................................................ 7

2.3 The Douglas- Peucker Line Simplification Algorithm .................................... 8

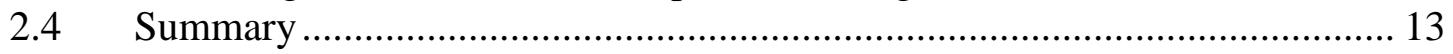

Chapter 3 - Systems Analysis and Design...................................................................... 15

$3.1 \quad$ Problem Statement ........................................................................ 15

3.2 Requirements Analysis ………………………................................... 15

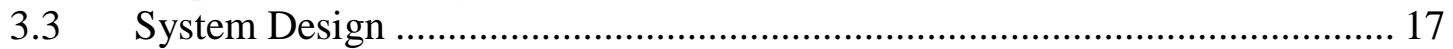

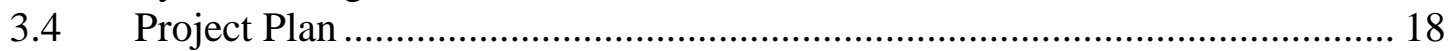

3.5 Summary .................................................................................... 19

Chapter 4 - Database Design................................................................................................. 21

$4.1 \quad$ Conceptual Data Model ............................................................................ 21

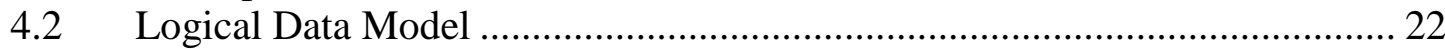

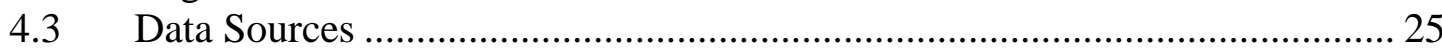

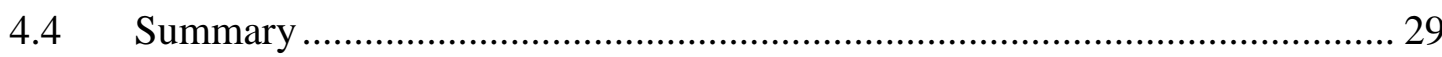

Chapter 5 - Implementation..................................................................................................... 31

$5.1 \quad$ Mapping Horizon Lines in Profile ................................................................. 31

5.2 Determining the Visual Significance of Horizon Peaks .................................. 39

5.3 Mapping Horizon Peaks in the XY plane ...................................................... 45

5.4 Aggregating Visual Significance scores to USGS Standard Peaks ................ 50

5.5 Representing the change in visual significance over time ............................... 55

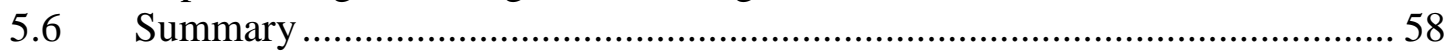

Chapter 6 - Results and Analysis..................................................................................... 61

Chapter 7 - Conclusions and Future Work ...................................................................... 63

Works Cited 65 



\section{Table of Figures}

Figure 1-1: Taller peaks are more visually significant. ...........................................2

Figure 1-2: Foreground peaks are more visually significant. ................................... 2

Figure 1-3: Steeper peaks are more visually significant. .......................................... 3

Figure 1-4: Isolated peaks are more visually significant. .......................................... 3

Figure 2-1: Douglas-Peucker Line Simplification Algorithm ................................ 10

Figure 2-2: Douglas- Peucker algorithm with smaller simplification tolerance........ 11

Figure 2-3: The Douglas-Peucker algorithm applied to a horizon line with ten

different simplification tolerance lengths. ......................................................... 12

Figure 2-4: The number labels represent the total number of times the Douglas-

Peucker algorithm selected for each vertex as significant. .................................... 12

Figure 3-1: System Design Diagram.................................................................. 18

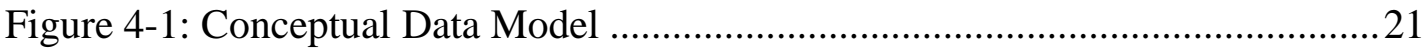

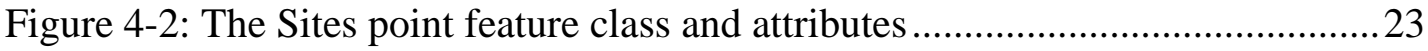

Figure 4-3: Horizon Lines table .......................................................................... 23

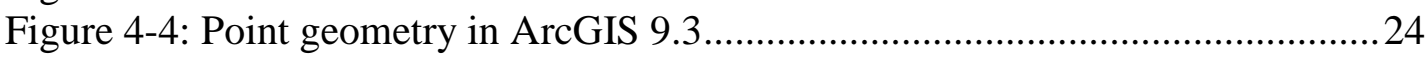

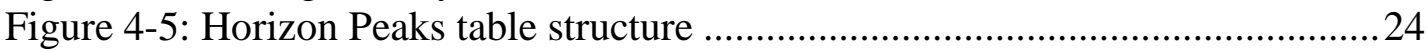

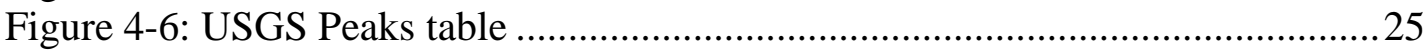

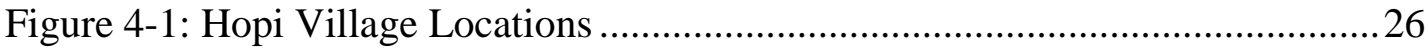

Figure 4-2: Hopi Horizon Lines.............................................................................. 27

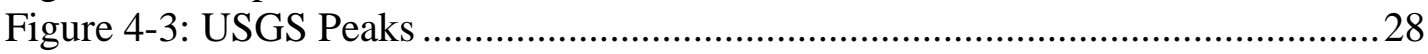

Figure 4-4: USGS Digital Elevation Model Mosaic............................................29

Figure 5-1: Horizon lines mapped in the cartographic perspective ......................... 32

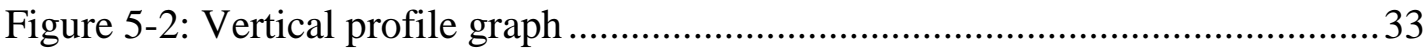

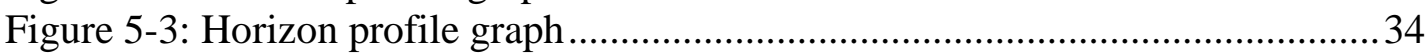

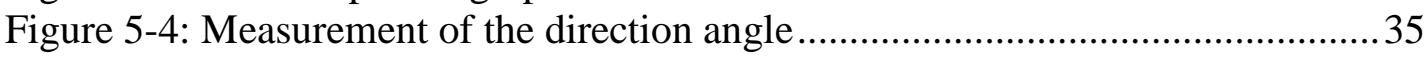

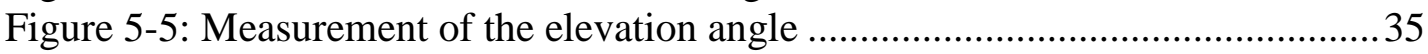

Figure 5-6: Horizon line mapped in profile and in the XY plane ............................ 36

Figure 5-7: Calculate Direction and Elevation Angles tool....................................... 37

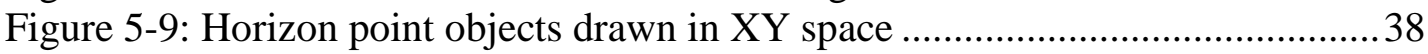

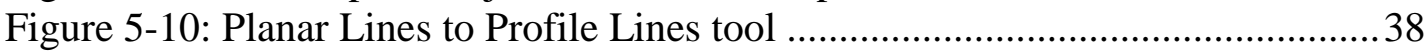

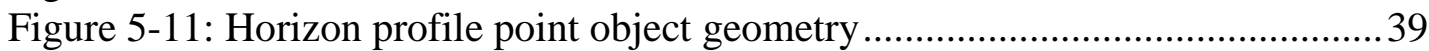

Figure 5-12: Using the Douglas- Peucker algorithm to select significant points ......40 Figure 5-13: Directional Angle Separation in the planar view is equal to the $\mathrm{X}$ Separation distance in the profile view............................................................... 41 Figure 5-14: Elevation Angle Separation is equal to the Y Separation distance in the profile view. ........................................................................................... 42

Figure 5-15: The final scores for all the vertices on one horizon profile. .................42

Figure 5-16: Horizon peaks and their visual significance count..............................43

Figure 5-17: The "Get Simplified Lines Tool" ................................................... 44

Figure 5-18: The "Count Significant Points" field ................................................... 45

Figure 5-19: The "Planar Lines to Profile Lines" tool...............................................46

Figure 5-20: Example result of planimetric horizon peaks and their visual significance count 
Figure 5-21: Example result of planimetric horizon peaks and their visual

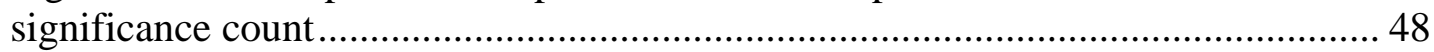

Figure 5-22: Example result of planimetric horizon peaks and their visual

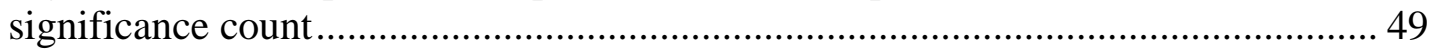

Figure 5-23: 400 meter buffers around USGS Peaks (black triangles). Horizon Peaks labels indicate the visual significance score that peak received with respect to the village that observed it. 51

Figure 5-24: Horizon Peaks within 400 meters of a USGS peak ............................ 51

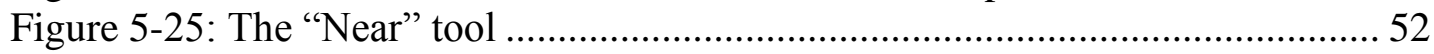

Figure 5-26: The "Aggregate to Standard Peaks" tool........................................... 53

Figure 5-27: Result of aggregating Horizon Peak visual significance scores to USGS

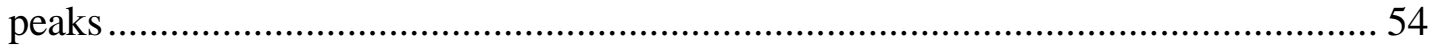

Figure 5-28: Total visual significance of USGS peaks ........................................ 55

Figure 5-29: Time interval attribute data for a Hopi village .................................. 55

Figure 5-30: Total visual significance of peaks from 1200-1250 c.e...................... 56

Figure 5-30: Total visual significance of peaks from 1251-1300 c.e...................... 57

Figure 5-31: Total visual significance of peaks from 1301-1350 c.e....................... 58 


\section{List of Tables}

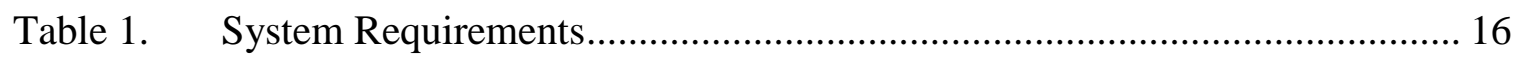






\section{Chapter 1 - Introduction}

This report describes the planning, design, and implementation of a project to solve a spatial problem as presented by Dr. Wesley Bernardini of the University of Redlands, Department of Sociology and Anthropology. Section 1.1 introduces the client and his background. Section 1.2 describes the problem the client seeks to solve as well as his role in the planning and design of the project. Section 1.3 describes the objectives, scope, and methods of the proposed solution to the problem. Section 1.4 describes the intended audience of this report. Section 1.5 provides a brief overview of the contents of the remainder of this report.

\subsection{Client}

The client for the Hopi Horizon Analysis project is Dr. Wesley Bernardini, a professor in the Department of Sociology and Anthropology at the University of Redlands. His academic research interests include studying the manner in which the Hopi culture retained knowledge of mountain peaks as they migrated to new places. Dr. Bernardini is an experienced user of Geographic Information Systems (GIS), having used GIS techniques in several past research projects. Prior to the beginning of the horizon analysis project, Dr. Bernardini had collected geographically referenced data for Hopi village locations, visual horizons, major mountain peaks of interest to his studies, and elevation data for the region of interest. Dr. Bernardini helped to define the criteria for analyzing the visual properties of horizon lines, defined the functional requirements of the proposed solution, and determined the critical success factors of the project. He also provided feedback on the preliminary results of prototypes as the project progressed.

\subsection{Problem Statement}

Dr. Bernardini wants to know in what way visual properties of landscapes can influence how people retain knowledge of distant places. Specifically, Dr. Bernardini wants to understand how the visual significance of mountain peaks on the horizon change when viewed by Hopi living in different village locations. Prior to this project's implementation, Dr. Bernardini had collected spatial data about Hopi village locations and their corresponding horizons, but he had not yet defined a method for quantifying the visual significance of horizon peaks with respect to the location of the observer. Dr. Bernardini wanted to use the method for quantifying visual significance of horizon peaks to help understand how the visual significance of certain peaks might change as the Hopi migrated to new locations over time.

\subsection{Proposed Solution}

The proposed solution to Dr. Bernardini's problem called for the implementation of a GIS with three major components: 1) a geodatabase for storing all data relevant to Hopi horizons, 2) geoprocessing tools to automate analytical methods performed on large datasets, and 3) a geovisualization environment for exploring the results of the analysis. 


\subsubsection{Goals and Objectives}

The first goal of the project is define a method for quantifying the visual significance of horizon peaks. This descriptor of a peak's visual significance must take into account how the horizon appears to an observer at a particular location. Some peaks will appear more or less visually significant depending on where the observer is located. The measure of visual significance must allow the ranking of peaks along the horizon based on how visually significant they appear. The client helped to define criteria for this measure of visual significance.

a) A peak that appears taller is more visually significant. Peak 2 is more visually significant than Peak 1.

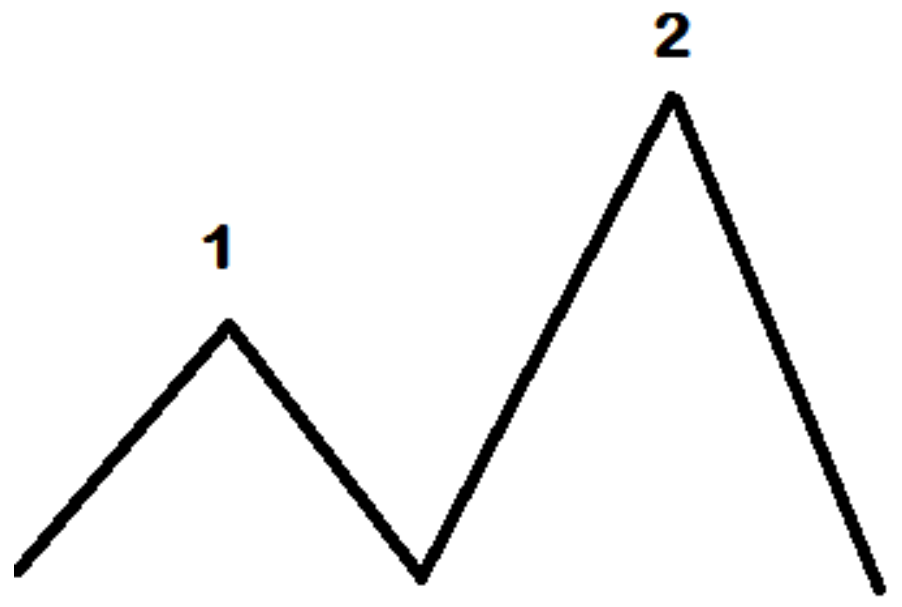

Figure 1-1: Taller peaks are more visually significant.

b) A peak that appears in front of another peak is more visually significant. Peak 1 is more visually significant than Peak 2.

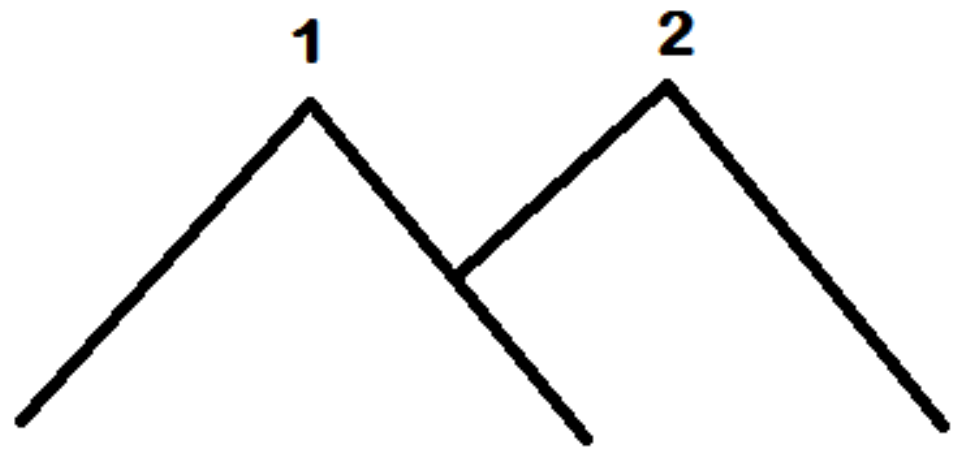

Figure 1-2: Foreground peaks are more visually significant. 
c) A peak that has a steeper slope on either side is more visually significant. Peak 2 is more significant than Peak 1.

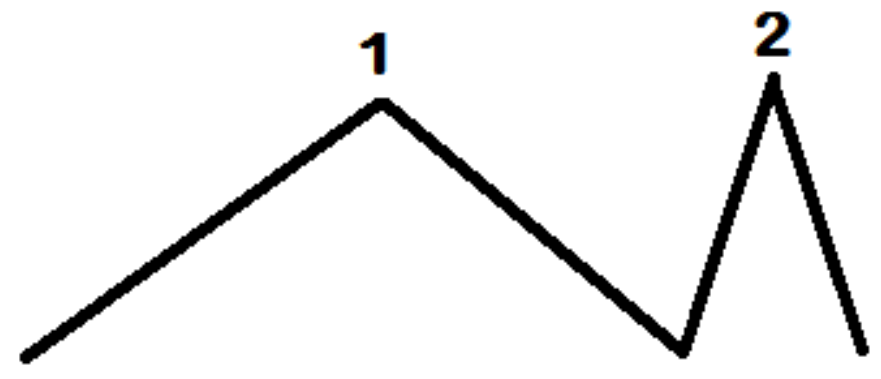

Figure 1-3: Steeper peaks are more visually significant.

d) A peak that is isolated from other similar peaks is more visually significant. Peak 2 is more significant than Peak 1.

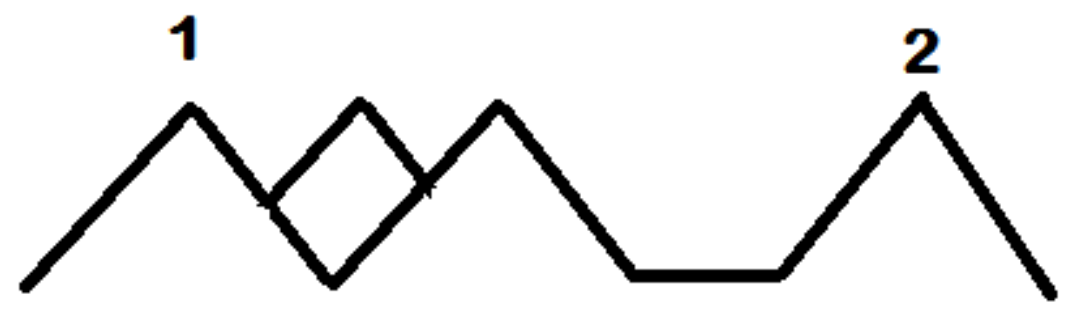

Figure 1-4: Isolated peaks are more visually significant.

Once a method for quantifying visual significance of horizon peaks had been determined, the next step was to assemble a geodatabase that will hold all of the information needed to calculate the visual significance. The geodatabase must be able to reliably store the data, support queries and retrieval of data, and allow the client to add updated data and the results of calculations.

After the geodatabase was built, the next goal was to develop geoprocessing tools that could automate the calculation of the visual significance of every peak on the horizon with respect to every Hopi village location in the study area. The geoprocessing tools must include a user interface that the client can operate intuitively. These tools must also be able to read and write data to the geodatabase.

The final objective of the project solution is to develop a geovisualization environment that allows the client to explore the results of the analysis. The geovisualization environment will support the mapping of features in the geodatabase. The most important objective is that the client will be able to use this environment to map the change in the visual significance of peaks in the study area over time. 


\subsubsection{Scope}

The timeframe for completion of this project was intended to occupy approximately 400 hours over a one year period. In this time, the client expected the following deliverables: 1) A geodatabase to store all relevant data and relationships, 2) A geoprocessing toolset for analyzing the visual significance of horizon peaks of each Hopi village, and 2) A geovisualization environment that supports the exploration of the change in visual significance of peaks over time.

A significant amount of the project time was spent designing the geodatabase, developing the geoprocessing tools, testing and debugging the tools, and creating methods for visualizing the results. Dr. Bernardini provided the source data for the analysis, helped define the criteria for ranking the visual significance of horizon peaks, and provided feedback on the results of prototype versions of geoprocessing tools and data visualizations.

\subsubsection{Methods}

The first objective for the project was the determination of a method for quantifying the visual significance of peaks. The client helped determine criteria that would affect how visually significant a peak would appear to a Hopi observer. Many different metrics for measuring visual aspects of topographic features were considered. Ultimately, the client agreed that an iterative approach using the Douglas-Peucker algorithm at varying levels of simplification was a good method for ranking peak significance that fit the criteria.

The development of the geodatabase and the geoprocessing toolset both required several rounds of building a prototype, testing, debugging, and refining. Each iteration of this process was driven by the development of the geoprocessing tools. Originally, there were only three geoprocessing tools built to work with the first version of the geodatabase. As new challenges in manipulating the data to support the analysis arose, additional tools had to be developed. These new tools would in turn require adjustments to the structure of the geodatabase.

The final aspect of the project involved creating visualization of the analysis results that revealed changes in peak significance over time. This stage of the project was only possible after the geodatabase and geoprocessing tools were complete. The geovisualization environment was the part of the project that occupied the least amount of time. The work for this stage involved using cartographic design principles to produce visualizations of the peak significance data produced by the geoprocessing tools.

\subsection{Audience}

This report is intended for an audience with a technical background in GIS as well as an interest in applying GIS practices to the domain of archaeology. The chapters in this report assume a familiarity with some basic principles of GIS. No background in archaeology is necessary for understanding the material covered here. 


\subsection{Overview of the Rest of this Report}

The remainder of this report provides detailed information about the Hopi Horizon Analysis project. Chapter 2 reviews the literature related to measuring visual properties of landscape features and previous work done in this domain. Chapter 3 explains the functional requirements of the system and gives a high-level description of the system design. Chapter 4 explains the source data used for the analysis, the project's conceptual data model, and the logical database design. Chapter 5 gives technical descriptions of the implementation of the project. Chapter 6 reviews the final results of the peak analyses. Chapter 7 concludes the report and highlights possible future work. 



\section{Chapter 2 - Background and Literature Review}

The main challenge of the Hopi Horizon Analysis project is determining a method for quantifying the visual significance of horizon peaks. Chapter 2 provides a background and review of previous work that has addressed the problem of quantifying the perceptual qualities of physical features. Section 2.1 describes work done concerning the spatial representation of visual aspects of landscapes. Section 2.2 reviews more specific approaches to quantifying the visual properties of mountain peaks. Section 2.3 describes the Douglas-Peucker line algorithm, a method for simplifying vector lines, and how it can be applied to the problem of ranking the visual significance of peaks on a horizon line.

\subsection{Visual Properties of Landscapes}

This project involves the application of visual analysis concepts and techniques in the context of archaeological research. On the subject of visual analysis, Llobera (2003) distinguishes between a visual property, a spatial representation, and a spatial configuration. A visual property is a measurable visual characteristic at a particular location in space and a spatial representation is the manner in which visual properties are stored and represented. The set of visual properties included in a spatial representation constitutes the spatial configuration. Different spatial representations generated from a particular visual analysis will account for different visual properties in their spatial configuration depending on the scope, scale, and intent of the problem (pp 30-32).

Once the visual properties and spatial representation for an analysis have been selected, the next challenge is to display the information in a meaningful way. Geovisualizations are visual displays of spatial information that allow exploration of the data, help generate new hypotheses, solve spatial problems, and construct knowledge (Kraak 2002, p 390). An effective geovisualization includes necessary data, excludes irrelevant data, and presents the user with an appropriate level of detail and degree of realism (Bodum 2004).

The study area for the visual analysis proposed for this project is the state of Arizona from 1100-1700 CE. During this period, Native American Hopi tribes migrated to different locations across the landscape. As groups migrated, the visual properties of their landscapes changed. Examples of visual properties that might be considered relevant to the Hopi visual experience are elevation, terrain, and landmark prominence. Representing how these visual properties change between different locations in a geovisualization environment is the central problem this project proposes to solve.

\subsection{Quantifying Prominence of Peaks}

Sinha (2008) wrote a dissertation on "The Delineation, Characterization, and Classification of Topographic Eminences." Sinha reviews more than 40 quantitative parameters that can be used to represent perceptual characteristics of eminences, including peaks. Sinha borrows a definition of "Topographic Prominence" from Maizlish (2003) as an eminence descriptor that satisfies all requirements of a good quantitative descriptor of eminences including that it is intuitive, objective, uniquely determinable, 
and easily quantified. Topographic prominence "is defined as the elevation of a summit relative to the highest point to which one must descend before reascending to a higher summit" (Maizlish, 2003). Christopherson (2003) used a GIS developed for the Umayri region of Jordan to compare topographic prominence between archaeological sites and suggest a link between the prominence of the sites and social hierarchy. Podobnikar (2009) developed a quantitative approach in a GIS environment for detecting the peaks of mountains in a digital terrain model. His quantitative method for peak detection was based in part on the measure of topographic prominence. Germino (2001) analyzed the visual properties of Rocky Mountain landscapes in both the planimetric and panoramic views to measure view quality.

An additional descriptor for quantifying the prominence of a peak is Omnidirectional Relief and Steepness (ORS). Earl (2010) describes the ORS method as correlating with the "visual impressiveness" of a peak by taking into account the relief and steepness of the peak.

\subsection{The Douglas- Peucker Line Simplification Algorithm}

Sinha (2008) explored some of the several factors that could determine what makes one peak more visually significant compared to another peak on the horizon. The client helped to list characteristics of a mountain that would make it visually significant in his area of research. The essential criteria for characterizing visual significance included: distance to the peak, the peak's apparent elevation, steepness of the slope on either side of the peak, and the relative height of that peak to nearby peaks. The client needed a method that would not just take into account physical properties of the peak itself, but how the perception of those properties is different for observers at different locations. There are several measures of peak significance that measure visual prominence.

Douglas and Peucker (1973) proposed a method for reducing the number of points stored in a digital line while still representing its "caricature" that eventually became known as the Douglas-Peucker Line Simplification algorithm. This algorithm is often used in GIS databases with large linear datasets, such as roads or streams. Saalfield (2009) used the Douglas-Peucker algorithm to detect and correct topological inconsistencies in GIS data. Visvalingam (1995) compared the Douglas- Peucker algorithm to an alternative line simplification algorithm by running the process on road datasets in a GIS at a variety of scales and tolerances. The objective of the algorithm is to reduce the number of vertices in a line by retaining only vertices that are most significant in representing the shape of the original line. The algorithm is an iterative method for selecting the vertices of a line that are most significant to the shape of the line and discards points that are less important.

The Douglas-Peucker algorithm has a simplification tolerance that determines whether a point should be retained or discarded. This simplification tolerance can also be conceptualized as the "maximum allowable offset" between vertices on the original line and the resultant simplified line (Esri, 2009). The larger the tolerance, the fewer points will be selected as visually significant and the resulting output will be a more generalized line. Smaller tolerances retain more points as visually significant and result in an output line that is a closer approximation to the shape of the original line. If the algorithm runs twice on the same line, at two different tolerances, some of the vertices on the original line will be selected for both runs, some points will be discarded by both, and other points 
will be selected during one run but discarded in the other. If the algorithm runs several times for several different simplification tolerances, it will select visually significant points selected more often than it will select less visually significant points.

Figure 2-1 illustrates how the Douglas-Peucker Line Simplification Algorithm works. The original line and its vertices are black. The length of the red line designates the length of the simplification tolerance. Green represents the trend line. The shape of the trend line changes as the algorithm progresses. The purple lines represent the offset distances between the vertices and the trend line. Vertices that are offset from the trend line by distance greater than the length of the simplification tolerance are circled in green. Vertices that are offset from the trend line by a distance less than the simplification tolerance are circled in red. The shape of the trend line when the algorithm finishes running becomes the final simplified line. The final simplified line is shown in blue. The process is as follows (Worboys, 2004):

a. Select the two end points of the original line and select them as visually significant. Draw the trend line segment between the endpoints.

b. Find the point between the endpoints that is the farthest from the initial trend line. If this point is offset from the trend line at a distance greater than the simplification tolerance, select the point as visually significant.

c. Redraw the trend line so that it connects the original endpoints to the point that was selected as significant in the first iteration. Find the two vertices between the endpoints of the trend line segments that are farthest from each segment. If the most distant vertex is farther from the trend line than the simplification tolerance, add it to the output point set and it becomes the next endpoint as the algorithm proceeds. If the vertex is closer to the trend line than the simplification tolerance, discard it and discard all the remaining points between the endpoints of that segment of the trend line.

d. Repeat the algorithm until all points are either selected or discarded. 

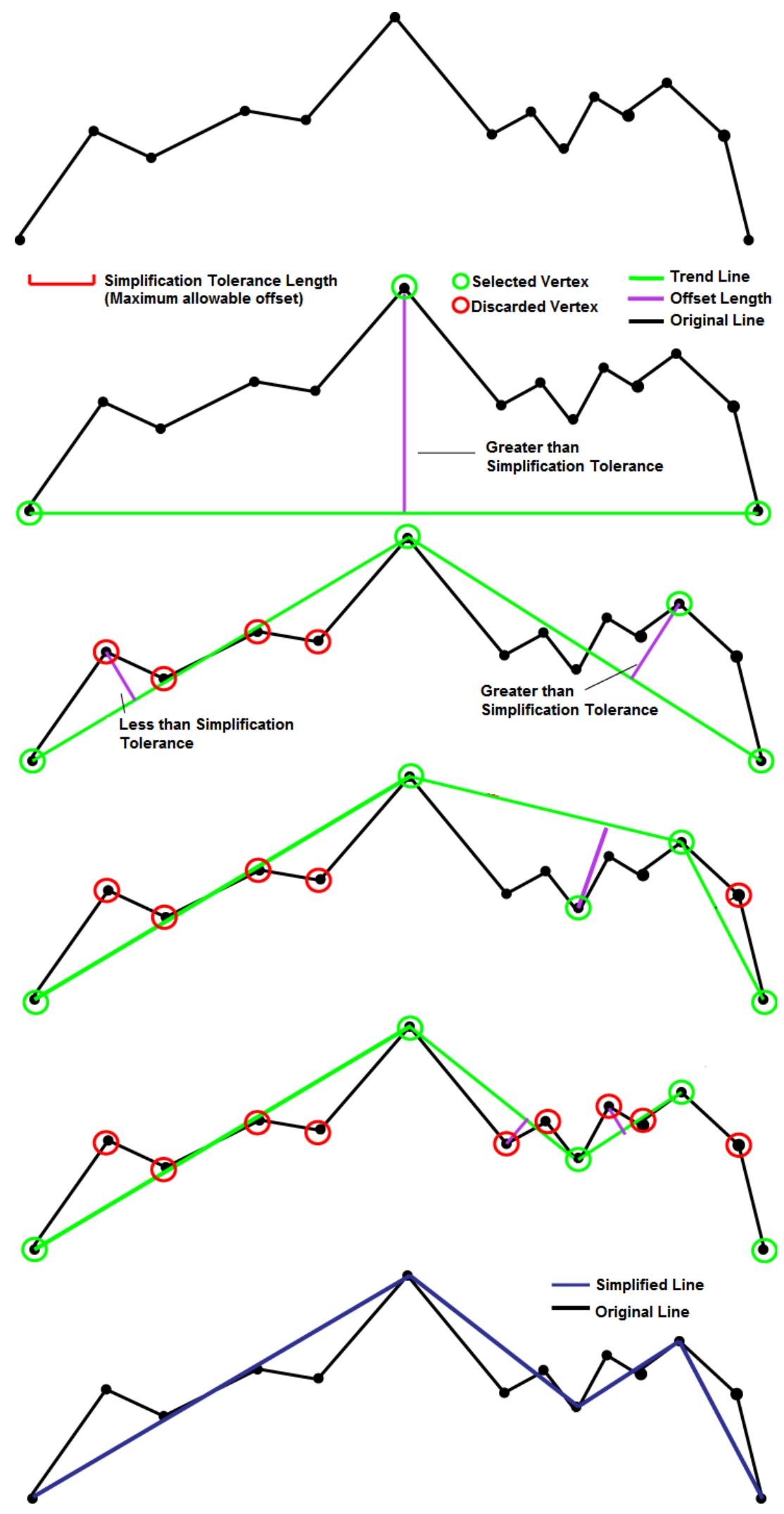

Figure 2-1: Douglas-Peucker Line Simplification Algorithm 
Figure 2-2 shows the result of the Douglas-Peucker Algorithm run on the same line with a smaller simplification tolerance. The end result of using a smaller simplification tolerance is that more points were chosen as visually significant than when using a greater simplification tolerance. The algorithm chose some of the points both times, some of the points only the second time, and did not chose the remaining points either time.

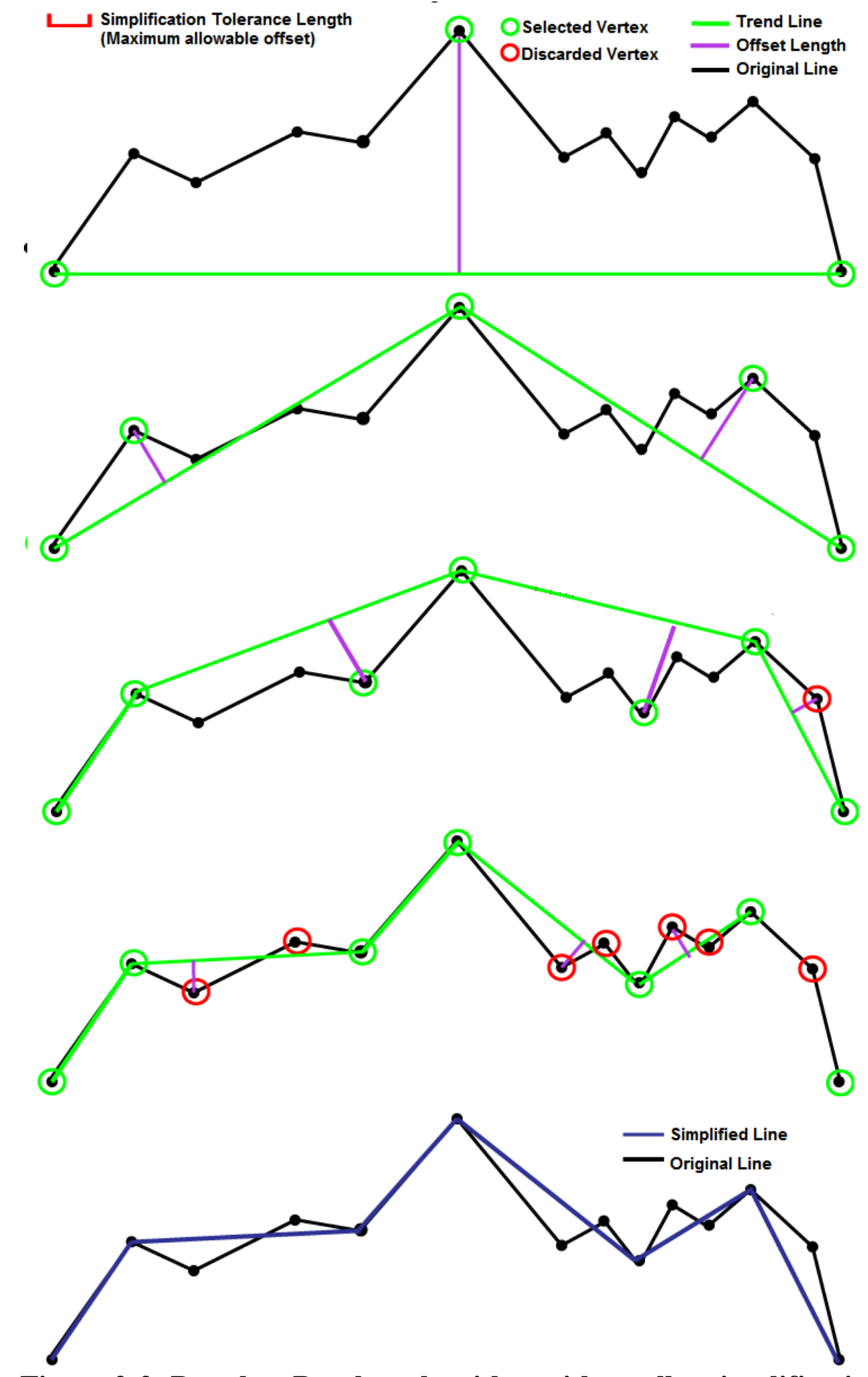

Figure 2-2: Douglas- Peucker algorithm with smaller simplification tolerance 
It is possible to run the algorithm on the line for many different simplification tolerances. If for each peak, the number of times it is selected by the algorithm is counted, that count would be an indicator of how visually significant the peak is on the line. If the line used were a visual representation of the horizon line as viewed by an observer, this iterative approach to measuring visual significance with the DouglasPeucker algorithm could be used to measure the visual significance of all the points on the horizon line. Figure 2-3 illustrates this method of applying the Douglas-Peucker algorithm on an actual horizon line for ten different levels of tolerance. Figure 2-4 displays the final count for each vertex (vertices that were selected zero times are not labeled).

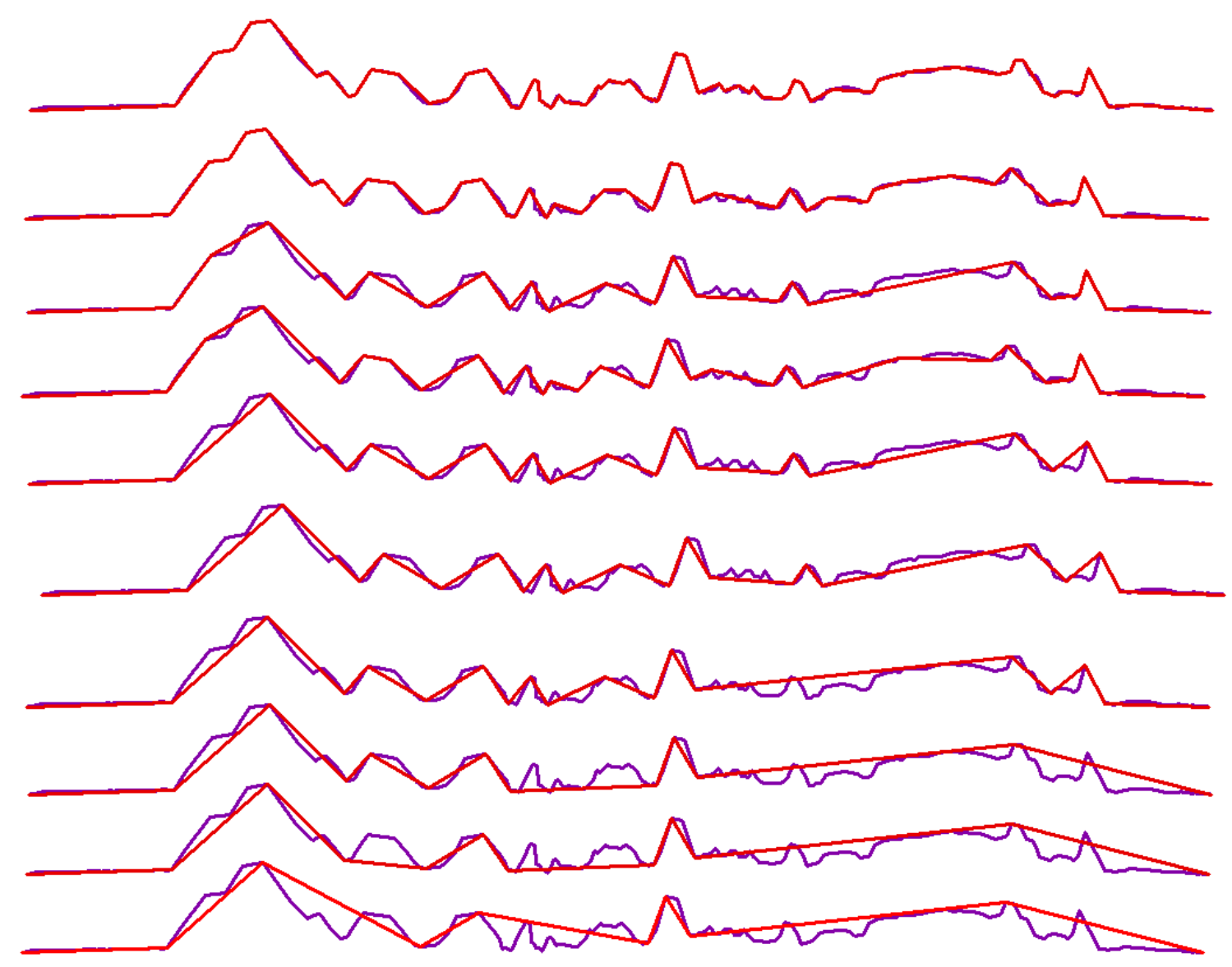

Figure 2-3: The Douglas-Peucker algorithm applied to a horizon line with ten different simplification tolerance lengths.

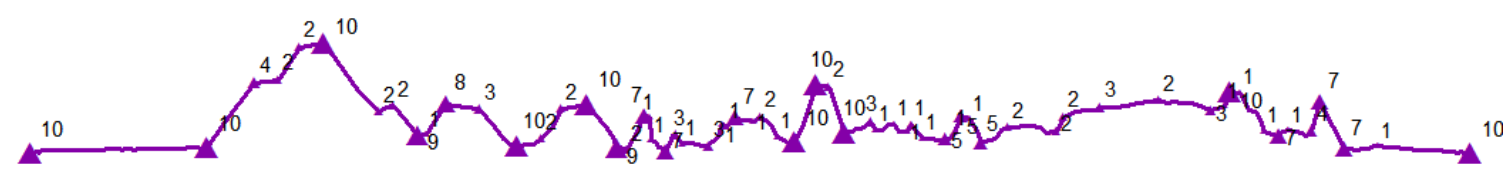

Figure 2-4: The number labels represent the total number of times the DouglasPeucker algorithm selected for each vertex as significant. 


\subsection{Summary}

Using GIS to explore and quantify visual properties of landscapes is an active area of research. Multiple attempts have been made to calculate visual prominence of peaks based on topographic features of the landscape itself. For the client's problem, the visual significance of peaks along a horizon line is what is important. A peak may occupy many different horizon lines and appear differently on each one. For this reason, an approach using the Douglas-Peucker algorithm at multiple simplification tolerances was chosen as the approach for quantifying visual significance. 



\section{Chapter 3 - Systems Analysis and Design}

This section provides a top-level perspective of the Hopi Horizon Analysis system design used to solve the problem of representing the change in visual significance of peaks on the horizon as they appear to observers at different locations. Section 3.1 reviews the problem the system attempts to solve. Section 3.2 analyzes the functional and nonfunctional requirements that the system design seeks to satisfy. Section 3.3 gives a description of the components of the system how they work together to fulfill the system requirements. Finally, Section 3.4 reviews the original plan for the project and its assumptions about the project and compares those plans and assumptions to the final system that was implemented.

\subsection{Problem Statement}

As Hopi tribes moved to new village sites over many years, the shape of the observable visual horizon changed. The visual significance of mountain peaks relative to other peaks on the horizon may have increased or decreased. New mountain peaks would appear and other mountain peaks would disappear from view. The client needs a systematic method for ranking visual significance of mountain peaks as viewed from the vantage points of a set of Hopi villages. The client then needs a way to aggregate the value an individual peak has for multiple observers who see it on their horizon during a given time period. Once this is known the client needs a way to visualize how each peak's significance either increases or decreases over time, as observer positions change. The problem involves comparing differences in the visual significance of common peaks on the horizon when viewed from different locations.

\subsection{Requirements Analysis}

The main requirements for the horizon analysis system are listed in Table 3.1. The client had done previous work with terrain and horizon analysis using the ArcGIS Desktop version 9.3 software. A developer had already created custom geoprocessing tools for him prior to this project, and the client was accustomed to loading data into these tools and viewing the results. For these reasons, the client requested a system that uses the ArcGIS Desktop for data storage, processing, and visualization.

The second major system requirement is a method for quantifying the visual significance of a peak on a horizon line as seen by an observer at a fixed location. The client has no previous method for calculating such a measure. The Douglas-Peucker line simplification algorithm defines a method for selecting the most visually important vertices on a given polyline at different simplification tolerances. The client agreed that the Douglas-Peucker algorithm could be reasonably applied to the horizon line problem to measure visual significance of mountain peaks.

After a method for measuring visual significance was determined, the system needed tools that would calculate this measure for each peak on each site's horizon. The client has too many Hopi sites and mountain peaks of interest in his study area for the desired calculations to be carried out by hand. The first tool automates the calculation of 
the visual significance of each mountain peak along each horizon for ever Hopi site. The second tool calculates the sum of these preliminary calculations, finds the USGS standard peak that each Horizon peak corresponds to, and sums the values. This calculation results in the total visual significance of a mountain peak for all villages in a selected time period.

Once the tools calculate the total visual significance of all the USGS mountain peaks and generate the resultant dataset, the client needs a way to visualize how the different mountain peaks either increased or decreased in significance across different time periods. The client would like to use the ArcMap interface to create maps of these values for fifty year intervals.

There are also two Non-functional requirements that the client would prefer to be realized but are not required. The first is that the automated geoprocessing tools can operate on the dataset as efficiently as possible. The second is that the tools will be compatible with any horizon line dataset, as long as it is in the ArcGIS geodatabase format so that the client can use the tools for future research projects.

\section{Table 1. System Requirements}

\begin{tabular}{|l|l|}
\hline System Requirement & Functional/Non-Functional \\
\hline $\begin{array}{l}\text { Use ESRI ArcGIS Desktop version 9.3.1 for } \\
\text { data storage and visualization }\end{array}$ & Functional \\
\hline $\begin{array}{l}\text { Use the Douglas-Peucker line simplification } \\
\text { algorithm for quantifying visual significance } \\
\text { of mountain peaks }\end{array}$ & Functional \\
\hline $\begin{array}{l}\text { Map horizon lines vertical profile } \\
\begin{array}{l}\text { Automate the calculation of the visual } \\
\text { significance of mountain peaks with respect to } \\
\text { multiple observer locations }\end{array}\end{array}$ & Functional \\
\hline $\begin{array}{l}\text { Automate the calculation of the total visual } \\
\text { significance of major peaks in the study area }\end{array}$ & Functional \\
\hline
\end{tabular}




\begin{tabular}{|l|l|}
\hline System Requirement & Functional/Non-Functional \\
\hline $\begin{array}{l}\text { Use the ArcMap graphic user interface to map } \\
\text { the change in the total visual significance of } \\
\text { major peaks over time }\end{array}$ & Functional \\
\hline $\begin{array}{l}\text { Automated processes run efficiently on a } \\
\text { virtual machine }\end{array}$ & Non-functional \\
\hline $\begin{array}{l}\text { Geoprocessing tools compatible with future } \\
\text { datasets }\end{array}$ & Non-functional \\
\hline
\end{tabular}

\subsection{System Design}

The Ancient Horizons and Landscapes system consists of components that support the implementation of a solution that will meet the functional requirements for the project. Figure 3-1 is a diagram of the system architecture. It consists of three functional components: a data Storage component, a data processing component, and a data presentation and visualization component.

The database component stores all geographic data that is used as input for the horizon analysis geoprocessing tools and visualizations. The database also stores data the output data produced by the operation of the geoprocessing tools. The client has a personal file storage location on the University's network file server that stores the database. The client can connect to the database from his desktop and retrieve data.

The geoprocessing component of the system executes the calculations on data in the database to generate the results of the horizon analysis. The geoprocessing component stores the scripts and the user interface tools that the scripts are accessed through. These scripted tools communicate with the database layer over the local network to retrieve input data. The geoprocessing tools process the data and store the results in the geodatabase. The client can also connect to the virtual machine remotely via his desktop personal computer. This allows the client to run data intensive tools on the virtual machine without monopolizing the functionality of his personal workstation for other tasks. This component will use the ArcGIS 9.3.1 geoprocessing framework and Python programming language version 2.5 .

The data presentation component of the system runs on the client's personal desktop. The desktop client runs ArcGIS Desktop 9.3.1. From here the client can explore the visual properties of the spatial data, create maps to publish or share, and retrieve information from the project database.

Each system component is capable of functioning independently of the others and communicates over the network. The geoprocessing component can pull data input from 
any database it points to, so in the future the client can run the analysis on a different dataset. The geoprocessing tools can also be run from on any machine that supports ArcGIS version 9.3. The geodatabase is portable and can be moved to an alternative storage location. It is also compatible with other tools that the client may wish to develop for future work. The database on the file server can be backed up to any location and may also be replicated and moved to the virtual machine, the client's desktop or any additional memory location. If the client wishes to share any of the data presentation elements with students or colleagues over the web, ArcGIS provides options for doing so.

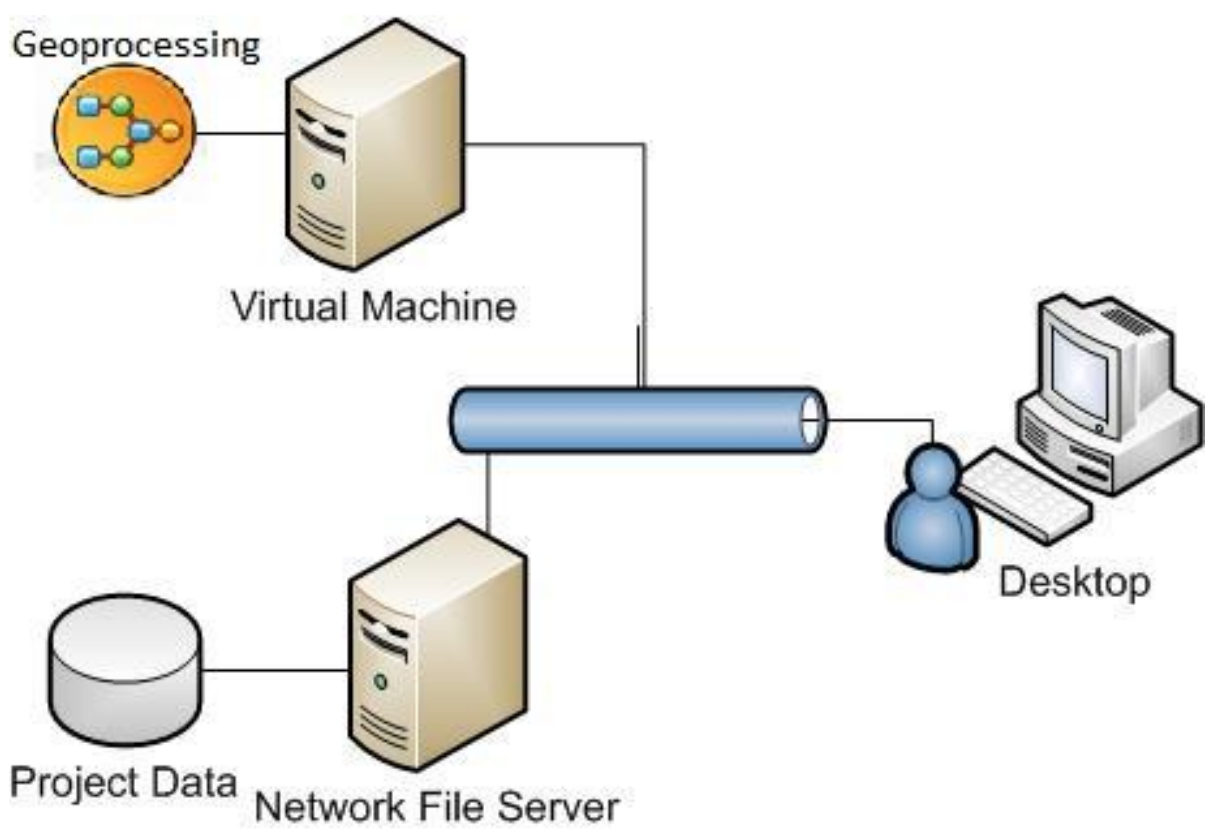

Figure 3-1: System Design Diagram

\subsection{Project Plan}

During the initial planning stages of the project, equal time was allotted to developing each component of the system. A shorter length of time was planned for researching methods for quantifying the visual significance of peaks. However, more time was spent researching existing methods for measuring the visual properties and then selecting a novel approach involving the Douglas-Peucker line simplification algorithm. Because the Douglas- Peucker algorithm had not been applied in this application domain previously, more time was spent developing geoprocessing tools to support the method.

The project plan had a much earlier time frame for developing the geoprocessing tools. At first, it was thought that one tool could perform the entire analysis. The method for processing the data and calculating the visual significance of every peak required more scripting than was anticipated.

The project assumed that it would be possible to automate all the processes involved in the calculation of peak visual significance. The project was successful in automating the entire process. The tools that were developed can be used in future analyses of visual significance. 
The project also assumed that using the Douglas-Peucker algorithm would work for selecting visually significant peaks along a horizon line. The algorithm was successful in ranking peaks that are significant to the shape of the line. However, it is not yet clear if the peaks that the algorithm selected were in fact the most visually significant in reality.

\subsection{Summary}

The Hopi Horizon Analysis system has three main components that satisfy the functional requirements of the project solution. The first component is the ArcGIS version 9.3 file geodatabase that stores the geographic data relevant to the analysis of Hopi horizons in. The second component is the geoprocessing element. The geoprocessing tools run the Douglas- Peucker algorithm, calculate visual significance of horizon peaks, and support the automation of the analysis for the entire dataset. The final component of the system is the presentation component that helps to visualize the change in visual significance of horizon peaks over time. 



\section{Chapter 4 - Database Design}

Chapter 4 of this report describes the source data used for the project, the conceptual design of the database, and the logical design of the database. Section 4.1 outlines the conceptual data model that explains how the system will represent the structure and relationships between the real world physical entities and their relationships. Section 4.2 covers the logical data model of the project database. This covers how the features and attributes are structured in the database. Section 4.3 explores the source data provided by the client and modifications to the dataset before it was ready to load into the database. The data includes the location of Hopi villages, the horizon of each village, mountain peaks, and elevation of the terrain.

\subsection{Conceptual Data Model}

The conceptual data model represents a high-level perspective of how the database abstractly represents the real world physical features. The conceptual diagram in 4-1 illustrates three essential entities modeled in the database, villages, horizons, and peaks.

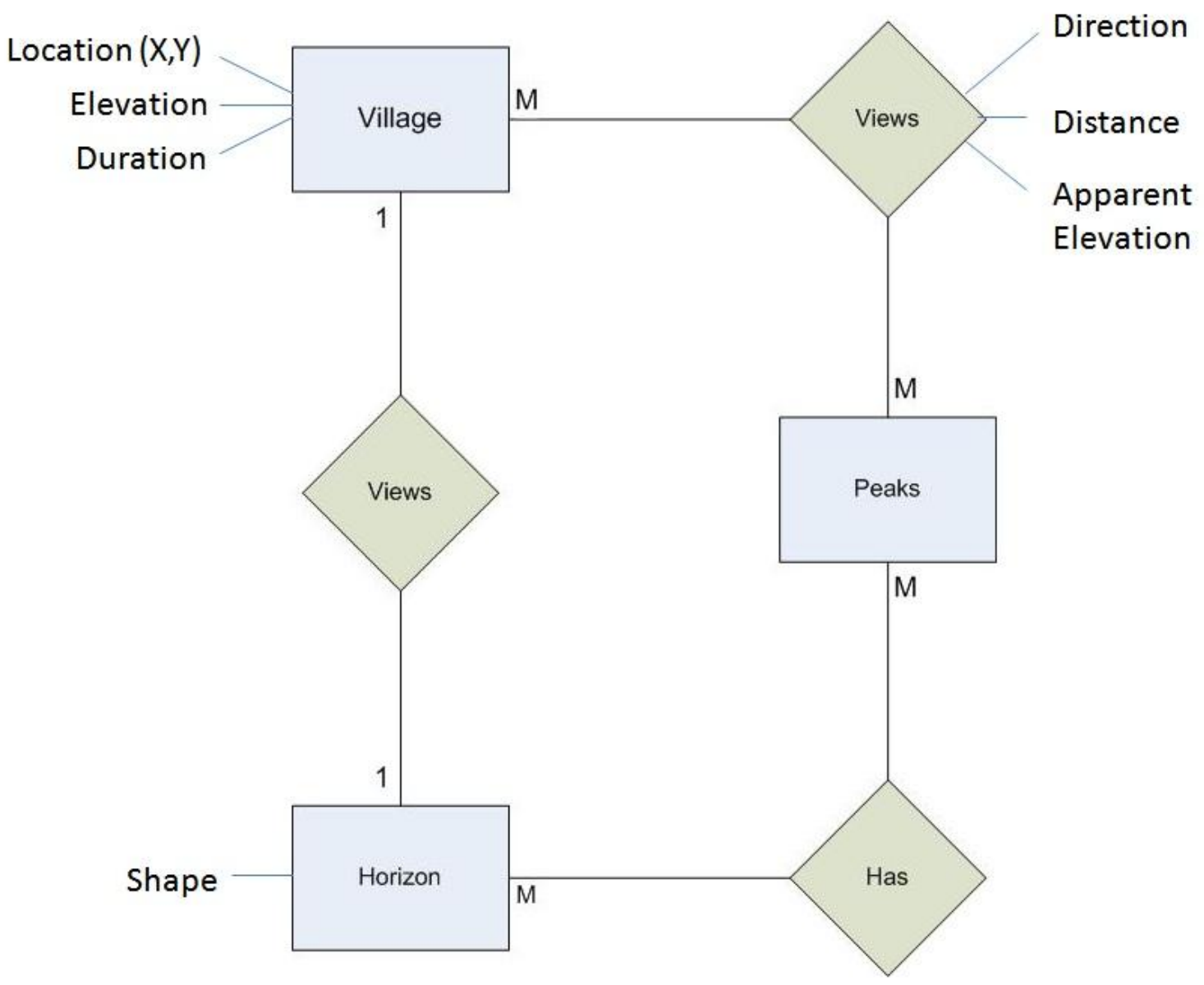

Figure 4-1: Conceptual Data Model 
In reality, the Hopi built villages in all three dimensions of space and existed for a specific span of time. For the purposes of the analysis, a Hopi Villages is simplified as a single point in space with an $\mathrm{x}$-coordinate (longitude), $\mathrm{y}$-coordinate (latitude), and a $\mathrm{z}$ coordinate (elevation). Time is an essential aspect to include in the model. Whereas a peak is permanently embedded in the terrain and does not move, the observers are able to move to new villages.

From these residences, the Hopi observed the surrounding landscape. A person standing in a village sees a uniquely shaped horizon. The horizon line forms the border between the surface terrain and the sky. From the perspective of an observer, a horizon forms a continuous, circular barrier between the visible terrain and the terrain that is hidden from view. Every Hopi village has one unique horizon.

The visual shape of the horizon consists of peaks, valleys, and ridges. Peaks are points on the horizon line that appear to have a greater height than the points nearby. Valleys are points on the horizon line that appear lower than surrounding points. Ridges are a sequence of points that appear to be near the same height, or rising at a gradual slope. The client is primarily interested in the peaks that lie along the horizon. Many peaks are visible from many villages. In addition, many peaks can be part of many different horizon lines. Each peak has a set elevation; however, its apparent elevation depends on its distance from an observer. Another attribute of interest for a peak is its direction relative to the observer.

\subsection{Logical Data Model}

The client is familiar with and regularly uses ArcGIS Desktop version 9.3.1. The client will also be the primary user of the system. For these reasons, an ArcGIS Desktop File Geodatabase was chosen as the format for the database. A single File Geodatabase can store all the data necessary for the functions required by the project. Also, because the storage capacity of all the project data is relatively small, the database can be copied, and moved to new locations, either on the client's network file server, personal desktop, or a virtual machine. The database consists of both source data provided by the client as well as the resulting datasets that the geoprocessing analysis produces.

The Hopi villages are modeled as a point feature class called "Sites" in the database. The structure of the Sites table is shown in figure 4-2. The Sites Feature class stores locations of Hopi villages and relevant information for each site. Each village has an $\mathrm{X}$ coordinate, a $\mathrm{Y}$ coordinate, and $\mathrm{Z}$ coordinate the represents elevation. These coordinates are stored in the "Shape" field. Each Site feature class has a unique field called "SiteID" of type "Short." Since each site has a unique horizon line, the SiteID connects each site in a one to one relationship with its horizon line. "OBJECTID." The remaining fields are titled with an underscore character and a year. Each of these fields represents a fifty year interval of time. These fields are a binary value. If the value in the attribute field is a " 0 " this indicates that the village was not occupied during that fifty year interval. If the value in the field is " 1 " that indicates that the village was occupied during that fifty year interval. 


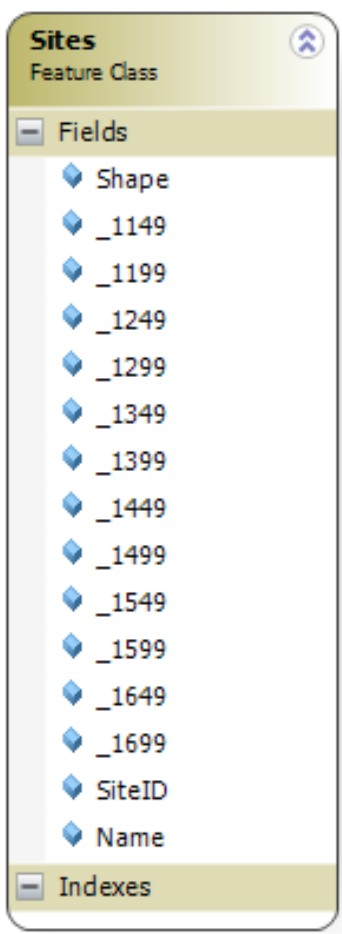

\section{Figure 4-2: The Sites point feature class and attributes}

The Horizon Line feature class connects the farthest points that an observer located in a given village center can see in every direction. The "SiteID" field relates the horizon line to its corresponding village.

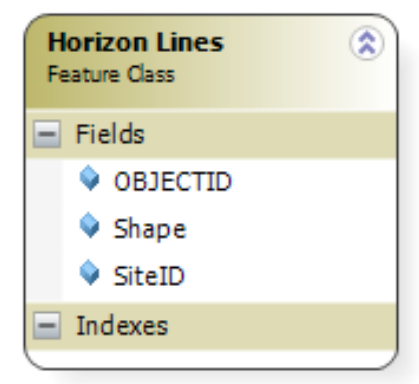

\section{Figure 4-3: Horizon Lines table}

Most of the relevant data for the horizon line is stored as geometry in the "Shape" field. In an ArcGIS geodatabase, a line is a vector feature that stores an array of point objects. Each point object has a mandatory $\mathrm{X}$ and $\mathrm{Y}$ attribute for storing coordinates. Additionally, a point object may store ID, Z, and M attributes. The ID attribute was used to as unique identifier for each horizon point, assigned in sequence. The data for this geodatabase is $\mathrm{Z}$ and $\mathrm{M}$ enabled. The $\mathrm{Z}$ attribute stores elevation of the point object. The DEM provided the elevation measure to assign to each point object's $Z$ attribute. The $M$ 
attribute was used to store the measure of the angle between the horizon vertex and the observer location.

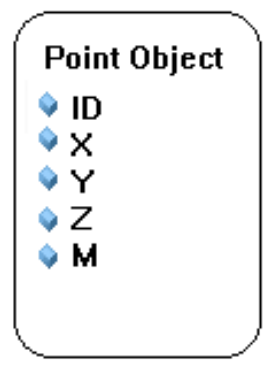

Figure 4-4: Point geometry in ArcGIS 9.3

The Horizon Peaks table is a point feature class. These Horizon peaks correspond to peaks on the horizon line. Each Peak has a "SiteID" to relate it to its horizon line. The "Count" field is the field that stores the visual significance of that peak. Although there is a one to one relationship between the horizon peaks and the horizons, in reality many of the points in the peak table represent the same point. However, each peak needs its own record for each horizon it appears on because it will have a unique visual significance score that is stored in the "Count" field. The "NEAR_FID" field identifies which point in the USGS Peaks table each horizon peak is nearest too. The "NEAR_DIST" field stores how far away the horizon peak is from its nearest USGS peak. These values are used to relate horizon peaks to one another. Every horizon peak that is within 400 meters of its nearest USGS peak is considered the same peak.

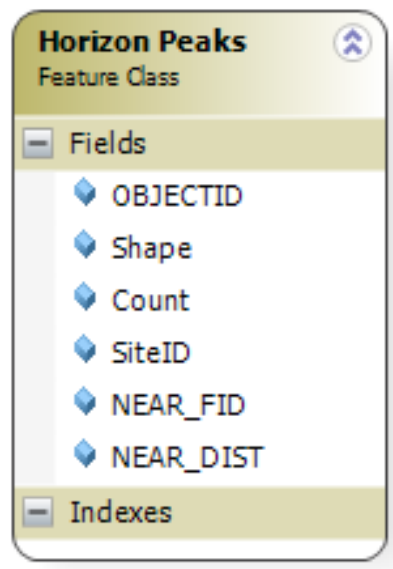

Figure 4-5: Horizon Peaks table structure

The "USGS Peaks" table stores data about peaks that were part of the USGS Global Names Information Service. These peaks are of interest to the client and are used to aggregate the "Count" scores in the Horizon Peaks table to the "Total Count" field in the USGS table. The "siteCount" field stores the number of sites that view that USGS 
Peak as a significant point on their horizon. The USGS Peaks table stores information about each peak's name, latitude, longitude, and height The USGS Peaks also have an "ORS_meters" value that indicates the omnidirectional relief and steepness value of that peak.

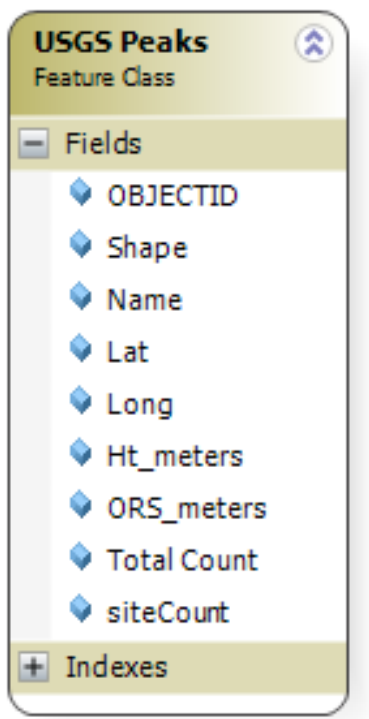

\section{Figure 4-6: USGS Peaks table}

\subsection{Data Sources}

The client supplied four datasets for the horizon analysis: Hopi village locations, the horizon line for each village, the locations of major peaks in the study area, and elevation data for the study area.

The Hopi village dataset is a point feature class that stores the coordinates of Hopi villages of interest to the client's time period. Each village also contains attribute data that is relevant to the client's research, including the name of the village and the name of the region where the village was located. The source data originally included 12 attribute fields of integer type each representing a fifty year interval between the years 1100-1700 c.e. The number stored in the field is the number of rooms in that village's dwelling during the fifty year interval. If the value is 0 , that indicates that the village was not yet in existence or no longer occupied during that time period. For the purposes of the horizon analysis, each fifty year interval could be recalculated as a binary value, with a "1" indicating that the village was inhabited during the time period and a " 0 " indicating that it was not inhabited. The SiteID field was also added to the village dataset as a unique identifier the villages with their horizon lines. 


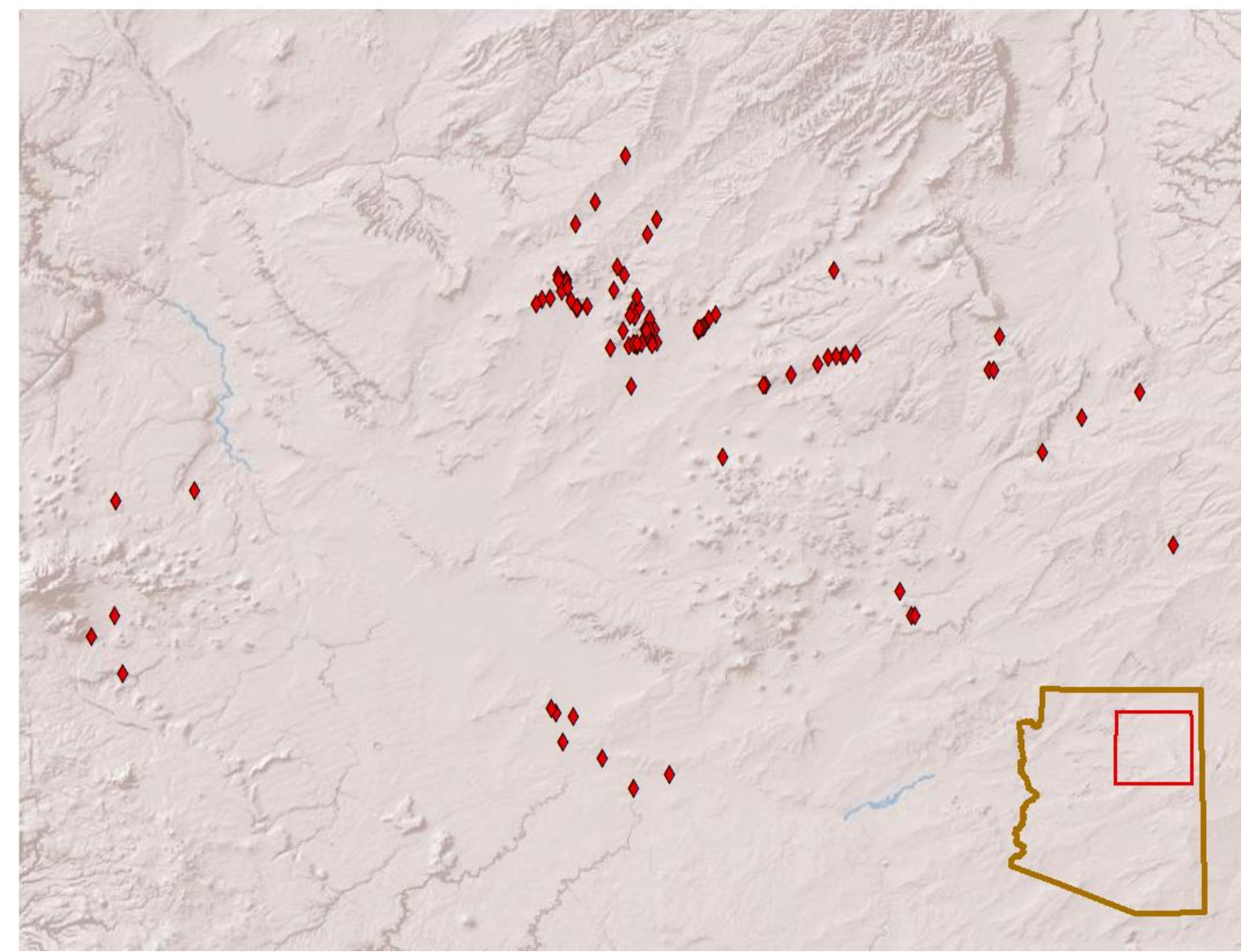

Figure 4-1: Hopi Village Locations

The client also provided Horizon line data for the test analysis. This horizon data was created from the web service "Hey, What's That?" This web service takes a point location input by the user and calculates the farthest point that is visible from every direction for an observer located at that point (Kosowski, 2010). The Redlands Institute created a custom tool for the client that allowed the client to submit a list of village locations, use this service to calculate the farthest visible point and then convert the output retrieved from the web service as lines in the ArcGIS version 9.3 feature class format. Figure 4-2 shows three example horizon lines and their corresponding villages. The horizon line was also given a unique "SiteID" attribute field to relate each horizon line with its village. 


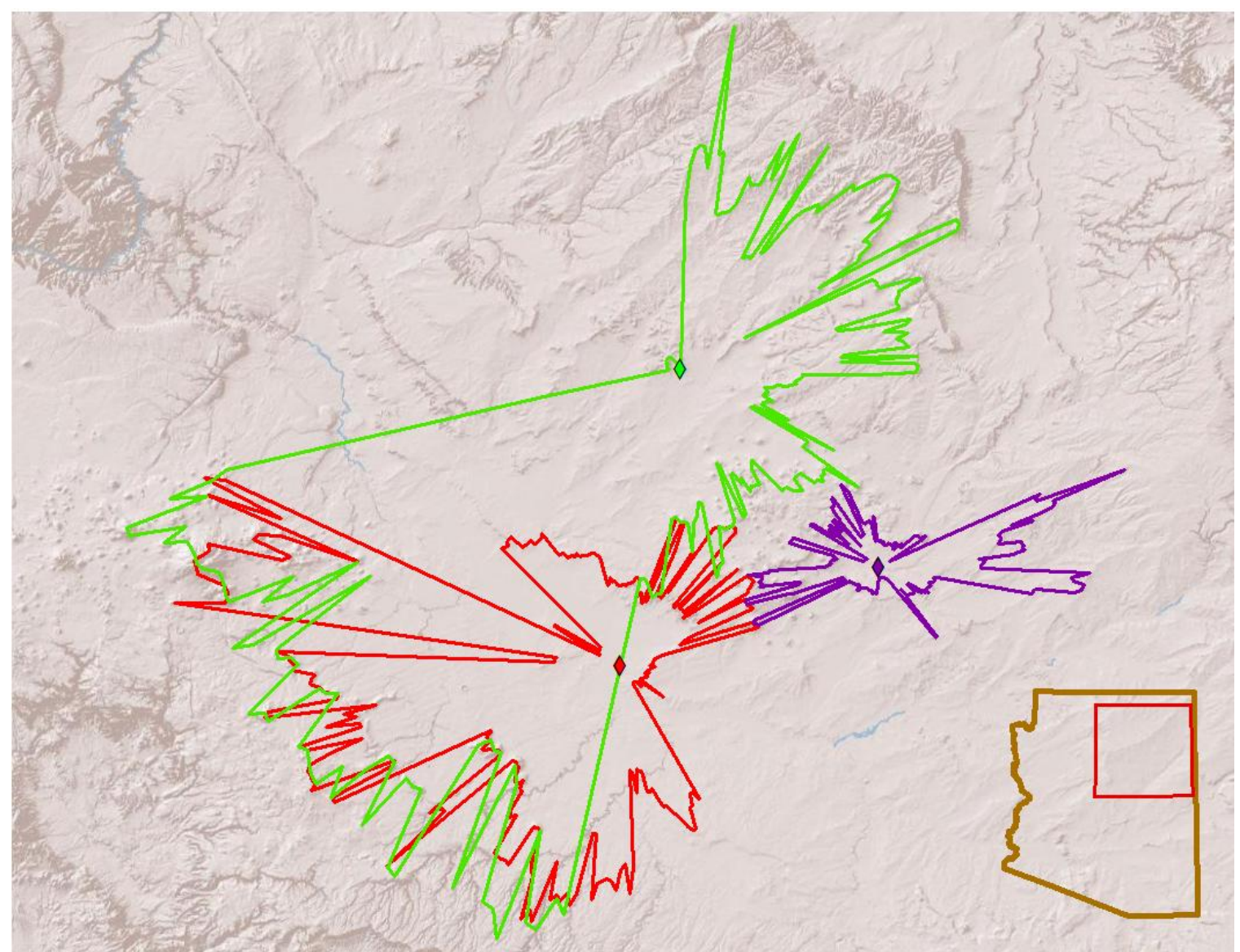

Figure 4-2: Hopi Horizon Lines

The USGS Peaks dataset provided by the client is a set of point location of peaks recognized by the USGS Geographic Names Information Service. These are all the point locations in the study area that the USGS has recognized as summits, ridges, or pillars. 


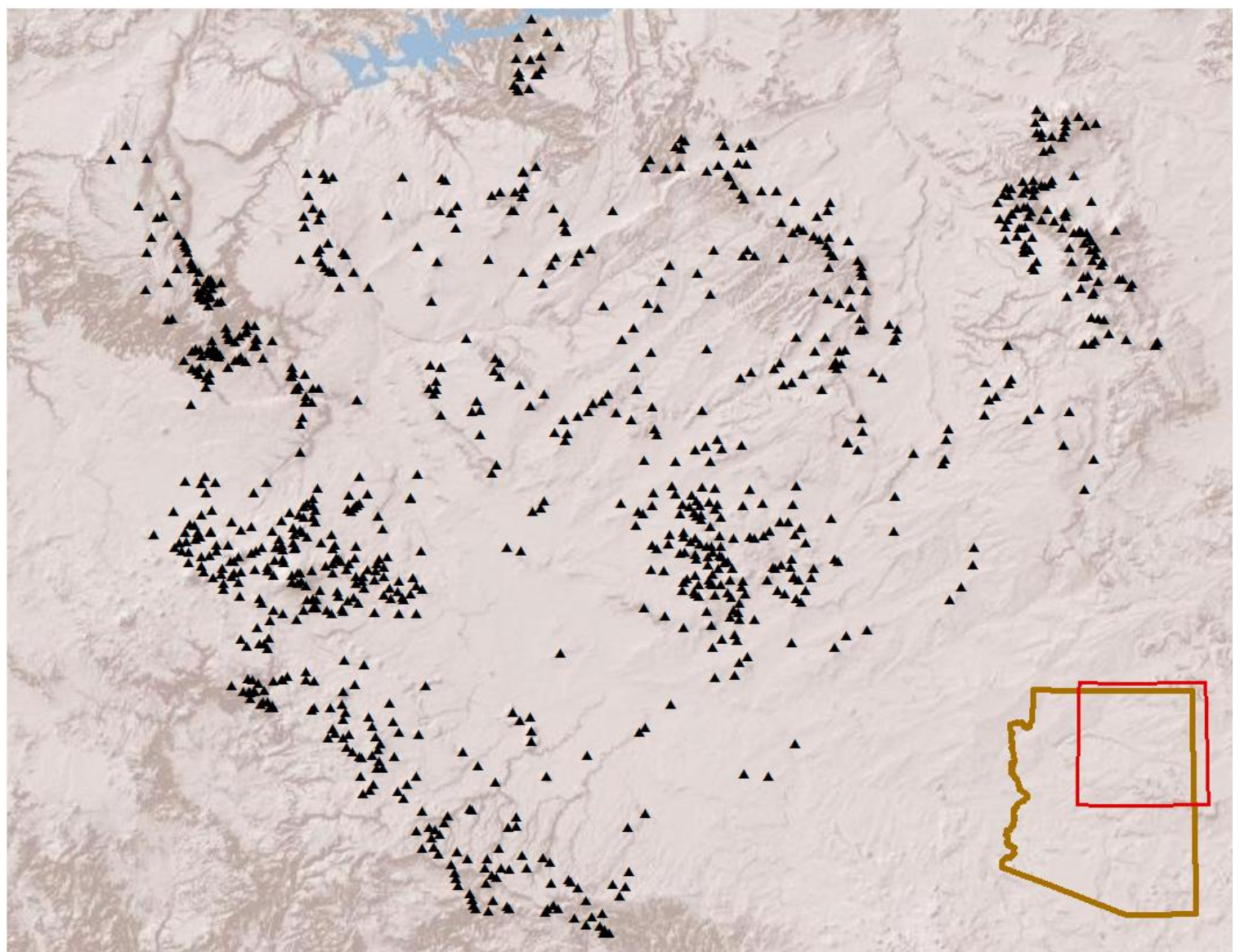

Figure 4-3: USGS Peaks

The elevation data provided from the client is from the USGS National Elevation Dataset. These data are in raster grid format, at a resolution of 30 meters. The original data were organized in 7.5 minute quadrangles in ArcGIS raster format. In order to use the DEMs for the analysis, the ArcGIS tool "Mosaic to New Raster" was used. This converted the individual DEMs into one seamless raster file. 


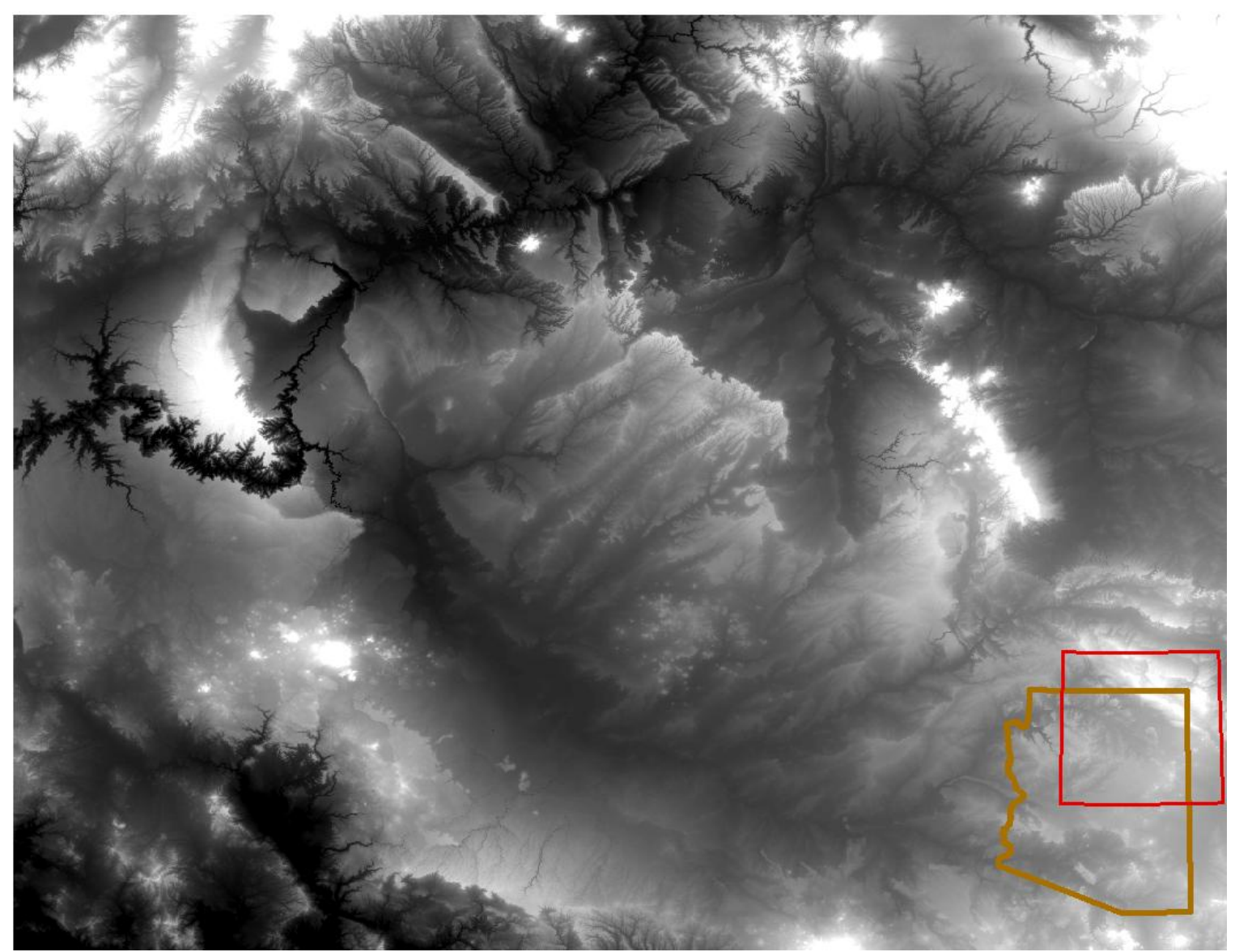

\section{Figure 4-4: USGS Digital Elevation Model Mosaic}

\subsection{Summary}

This chapter describes the structure of the geodatabase used to organize and store data in support of the Hopi Horizon Analysis. The geodatabase stores essential data concerning the Hopi villages, the horizon lines of every Hopi villages, and the horizon peaks that belong to each horizon line. Additionally the geodatabase stores the "SiteID" field for each of three features that connect each village to its horizon line and each horizon line to its horizon peaks. This data is the foundation for implementing the horizon analysis tools described in Chapter 5. 



\section{Chapter 5 - Implementation}

Chapter 5 of this report describes how the components of the system were implemented to perform the analysis. Each section addresses a functional segment of the analysis

workflow. Section 5.1 describes the problem of mapping horizon lines in profile and the tools developed to perform this task. Section 5.2 details the calculation of the visual significance of peaks on the horizon profiles. Section 5.3 discusses how the horizon peaks are mapped back to XY planimetric space after the calculation of their visual significance. Section 5.4 explains how the visual significance of horizon peaks was aggregated and applied to the USGS peaks dataset. Section 5.5 illustrates how the mapping of the change in the aggregate visual significance of peaks over time. Finally, Section 5.6 summarizes the entire project workflow from start to finish.

\subsection{Mapping Horizon Lines in Profile}

The horizon lines provided by the client have $\mathrm{X}$ and $\mathrm{Y}$ coordinates and can be mapped in the XY coordinate plane from a planimetric perspective. Figure 5-1 shows three example Hopi village locations and their corresponding horizon lines. This view is useful for determining the relative distance of points along each horizon, however it does not reveal how the peaks appear to the observer viewing the horizon from a village. In order to visualize the perspective view of the horizon to an observer, the horizon must be mapped in profile. 


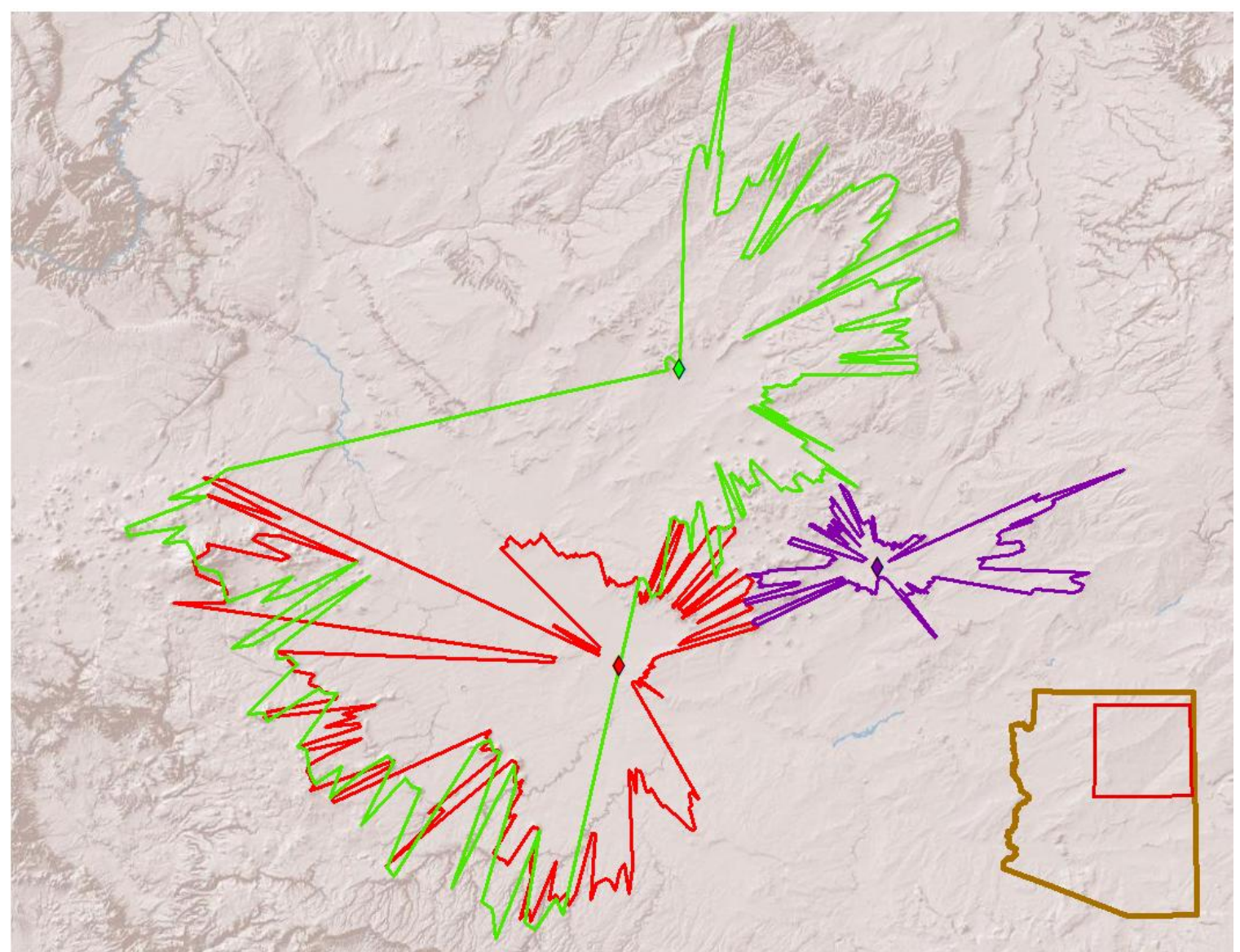

Figure 5-1: Horizon lines mapped in the cartographic perspective 
Profile maps of terrain are often used to map features in a way that emulates the view of those features from the ground. A vertical profile map, shown in Figure 5-2, is one technique for mapping features in profile. In a vertical profile map, a path is drawn across the terrain, from a start point at 0 units of distance, to an end point. The $\mathrm{X}$ axis represents the distance along the path and the $\mathrm{Y}$ axis represents the elevation of the terrain. The vertical profile view is useful for visualizing the shape of the terrain along a given path and the slope of the terrain along that path. However, the vertical profile still only offers the vertical perspective of the terrain for somebody walking along that particular path. It does not map the visual properties of the terrain for observers viewing the landscape from a distance. In figure 5-2, Peak $A$ has a height of $y_{1}$ meters and is at a distance of $x_{1}$ meters along the feature path. Peak B is farther down the path than Peak A, and has a much greater absolute height $\left(\mathrm{y}_{2}>\mathrm{y}_{1}\right)$. This vertical profile graph is useful for comparing absolute elevations and slope differences, but does not take into account how an observer's distance from different points on the line affects the apparent height of those points. Peak A may appear to have a higher altitude to an observer who is near to Peak A and far from Peak B

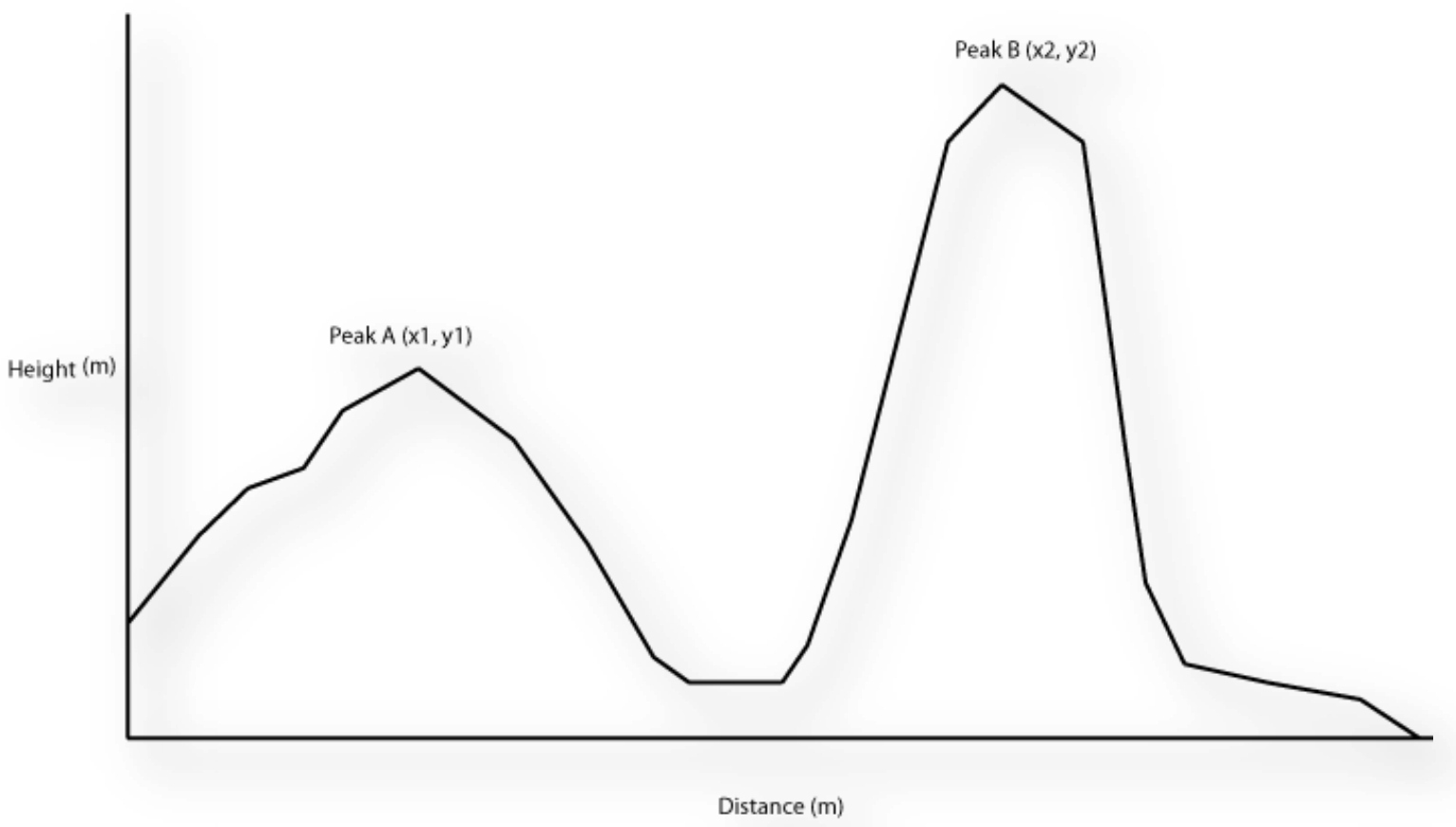

Figure 5-2: Vertical profile graph

Figure 5-3 is a profile graph of a horizon line where the $\mathrm{X}$-axis represents the "Direction" in degrees between each vertex on the horizon line and the Y-axis represents the "Elevation Angle." This representation of the horizon line is a better approximation of the shape an observer sees when viewing the horizon. A ground observer sees a boundary between the horizon and the terrain in 360 degrees. The value of this direction angle can be mapped on the X-axis for each point along the observer's horizon. On the Y-axis is a measure of the angle between the observer's visual plane and the line of site to each point 
on the horizon line. The greater the elevation angle, the higher a point appears from the perspective of the observer.

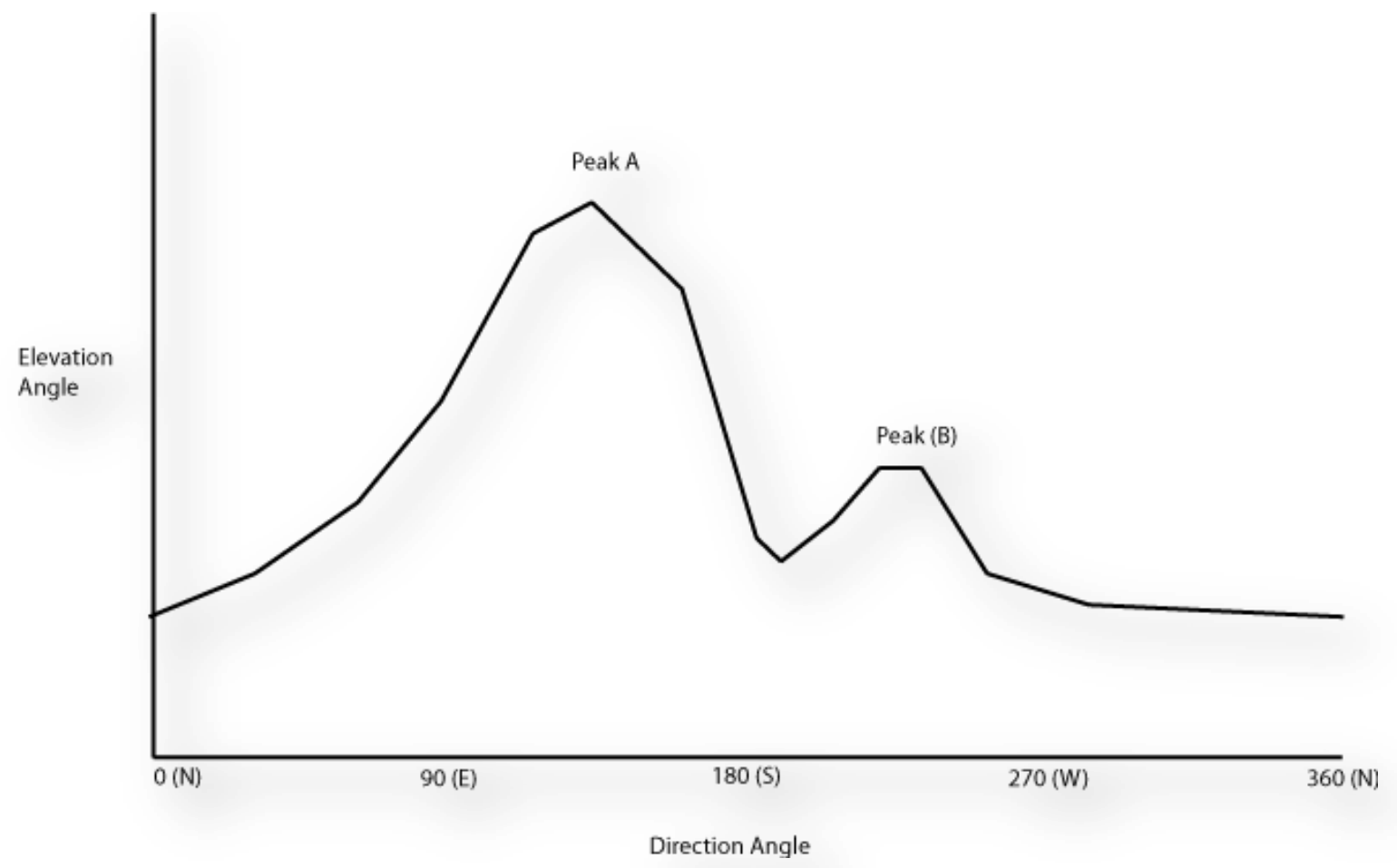

Figure 5-3: Horizon profile graph

Figure 5-4 illustrates the measurement of the direction angle. The observer location is the origin of the coordinate frame. North is arbitrarily chosen as 0 degrees, East is 90 degrees, South is 180 degrees, and West is 270 degrees. For each peak, a direct line, or "line of sight," is drawn to the observer origin. The direction is the angle between the North axis and the line of sight. 


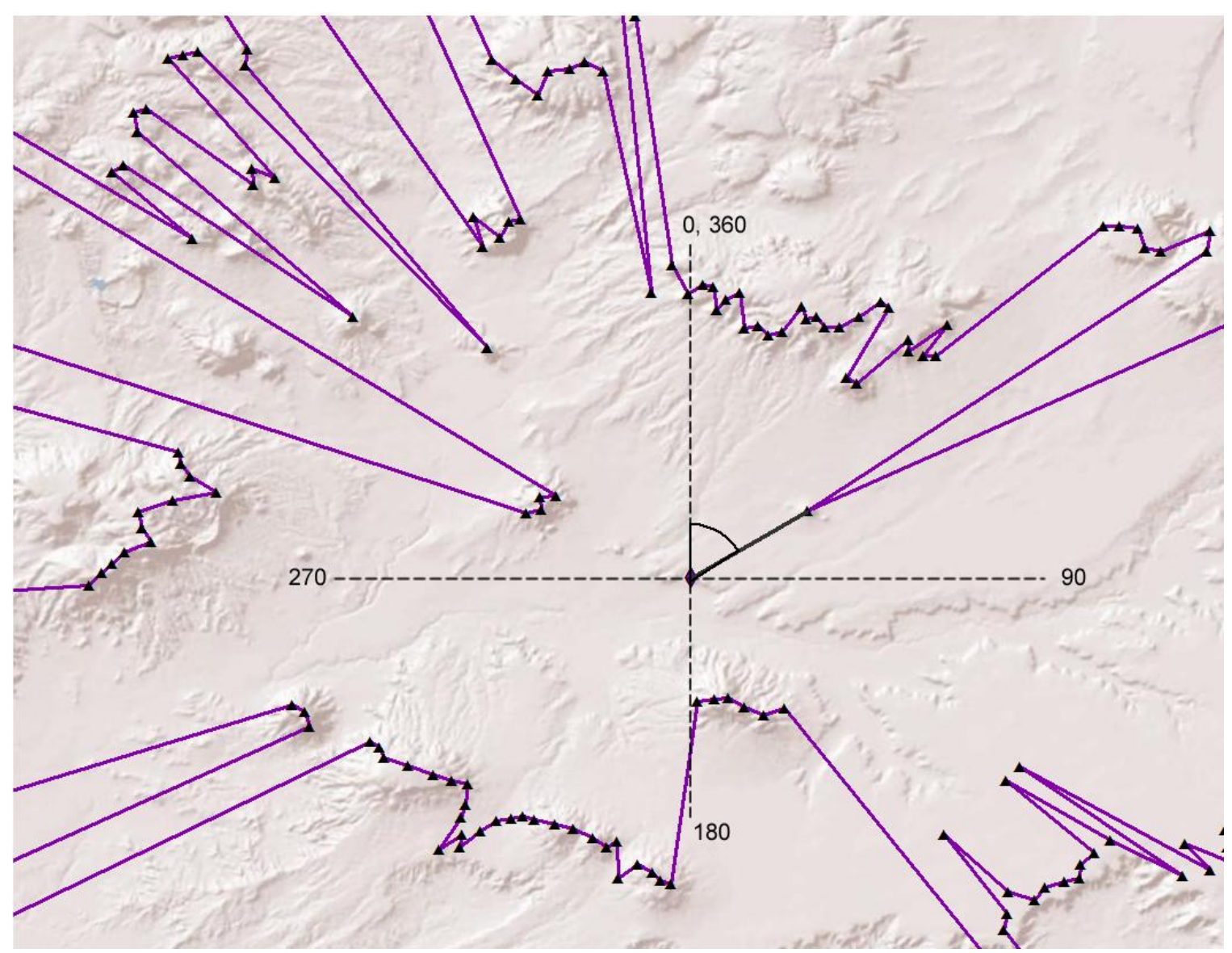

Figure 5-4: Measurement of the direction angle

Figure 5-5 illustrates the measurement of the elevation angle between a peak and an observer. It is the angle between the line of sight from the observer and the line tangent to the observer's visual plane.

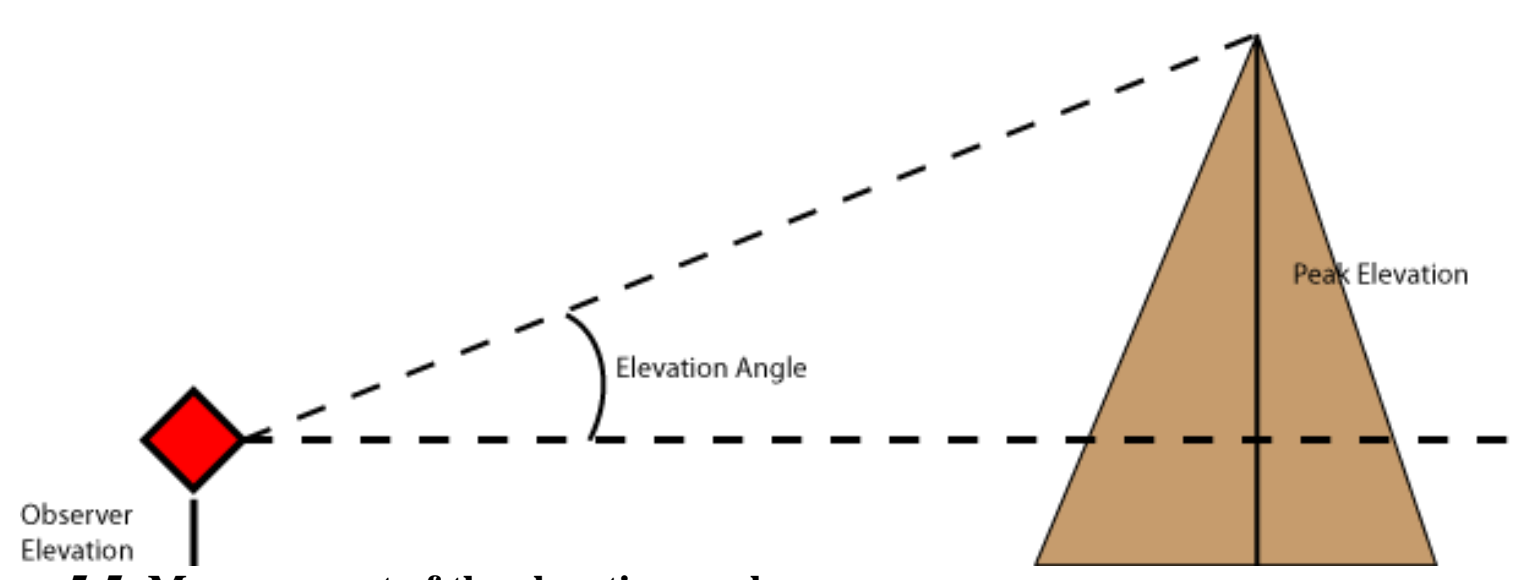

Figure 5-5: Measurement of the elevation angle

Figure 5-6 shows an example horizon lines mapped in the XY plane and its corresponding horizon profile mapped as direction angle vs. elevation angle. 


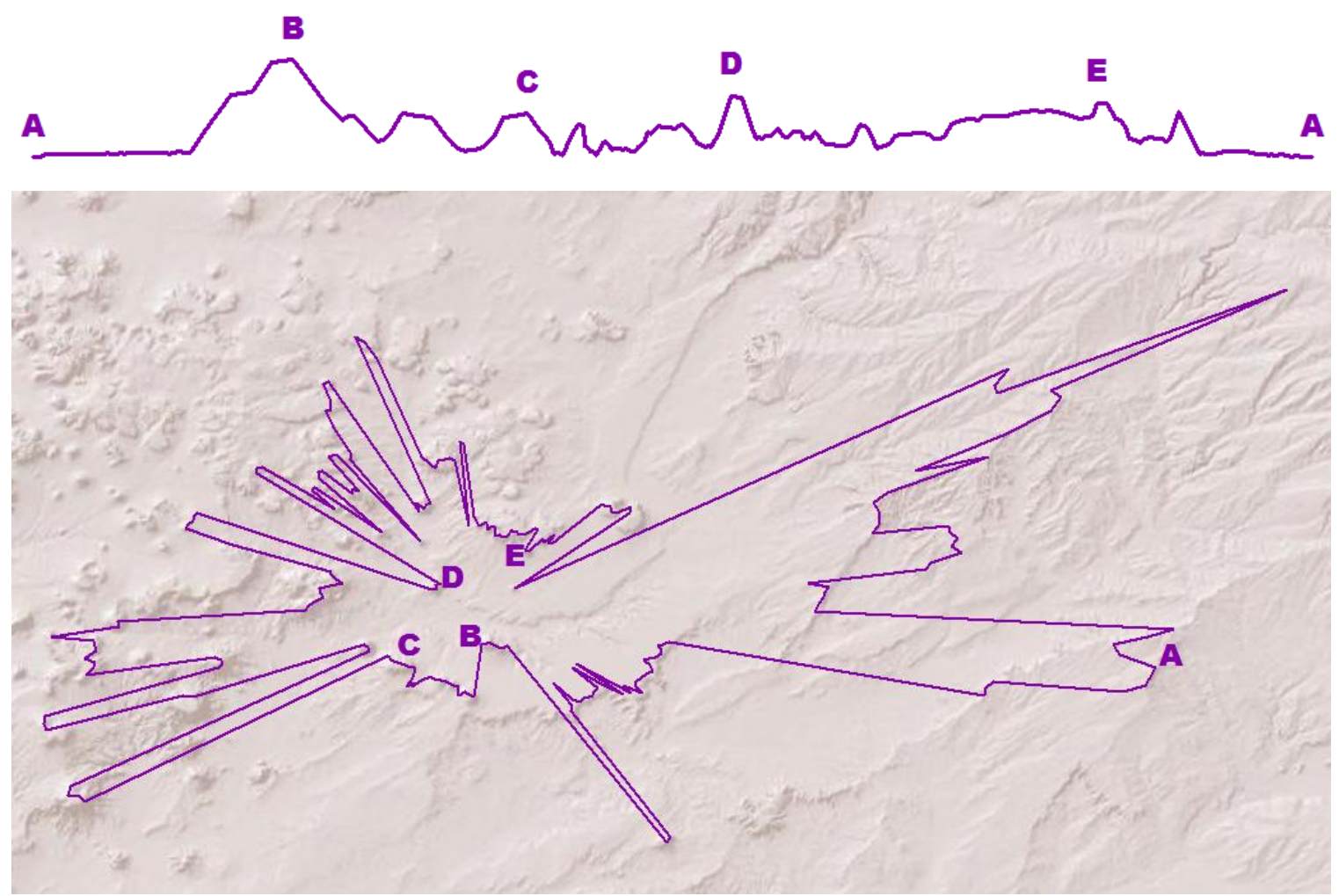

Figure 5-6: Horizon line mapped in profile and in the $X Y$ plane

The "Calculate Direction Angle and Elevation Angle" is a script written in the ArcGIS geoprocessing environment for the Python version 2.5 scripting language. Figure 5-7 shows the interface for using the script tool in ArcGIS Toolbox. The tool accepts any line feature class in the ArcGIS 9.3 file geodatabase format as input in the first line. The second line accepts a point feature class. Both the lines and the points must have an attribute field called "SiteID" this is used to pair each horizon line with its respective village site. The Elevation Surface field takes a DEM in raster format as input. The "Output File" field is the file path for the output feature class. 


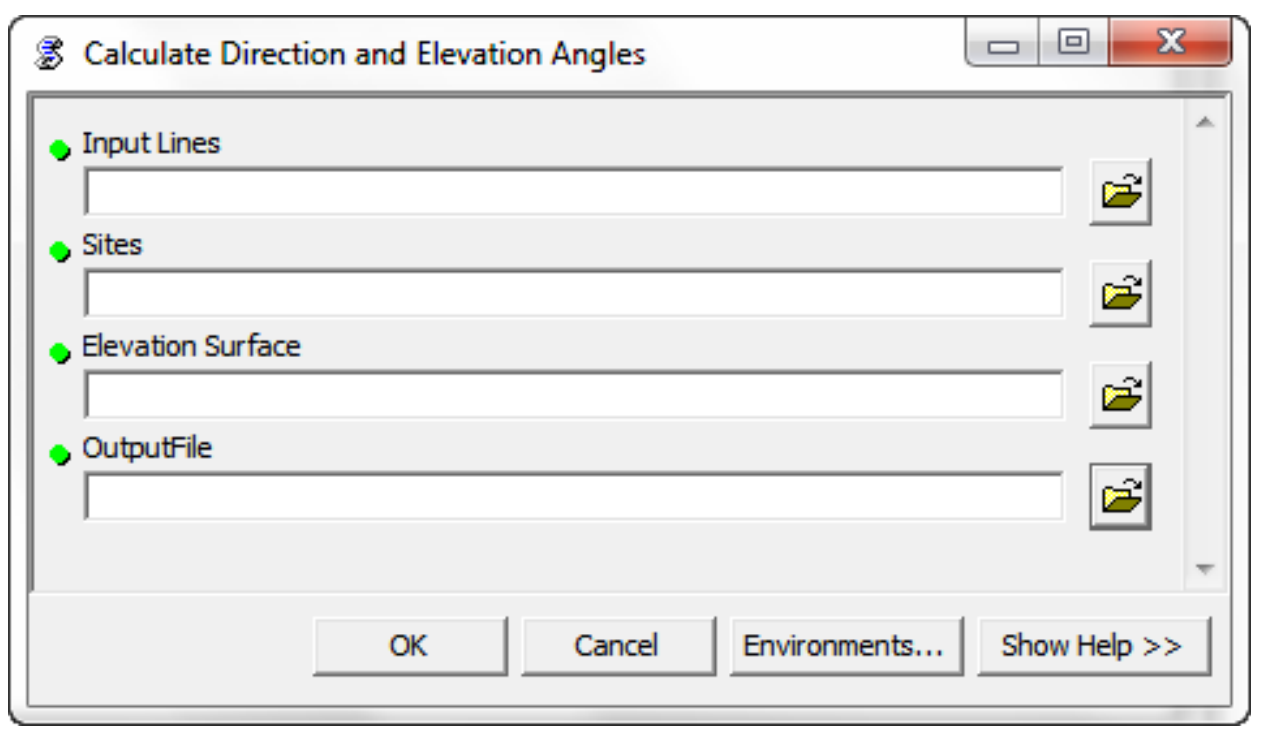

Figure 5-7: Calculate Direction and Elevation Angles tool

The output of this tool stores the elevation angle and direction angle of every vertex in the "Shape" field. The Shape field stores the geometry of each horizon line as an array of point objects. Each point object has five attributes: ID, X, Y, Z, and M. When ArcMap renders the line on the map display, it draws the lines that connect these points together in $\mathrm{XY}$ space, based on their order in the array. The $\mathrm{X}$ attribute is drawn as the $\mathrm{X}$ coordinate and the $\mathrm{Y}$ attribute is drawn as the $\mathrm{Y}$ coordinate. The $\mathrm{Z}$ attribute is most often used to store elevation values, but can be used to store any floating number type to represent a third dimension of geographic data. The $\mathrm{M}$ attribute is most often used in linear referencing applications, to store "measures" of a quantity along a line. In this application, our goal is to visualize the horizon line in profile with the $\mathrm{X}$ axis as Direction angle and the $\mathrm{Y}$ axis as Elevation angle. The Calculate Direction and Elevation Angle reads the $\mathrm{X}$ and $\mathrm{Y}$ attributes of each horizon line and calculates the direction angle between those coordinates and the coordinates of the corresponding village. This direction angle is stored in the "M" attribute field of that vertex's point object. The script then calculates the elevation angle between the vertex and the village and stores this in the "Z" attribute of that vertex's point object. 


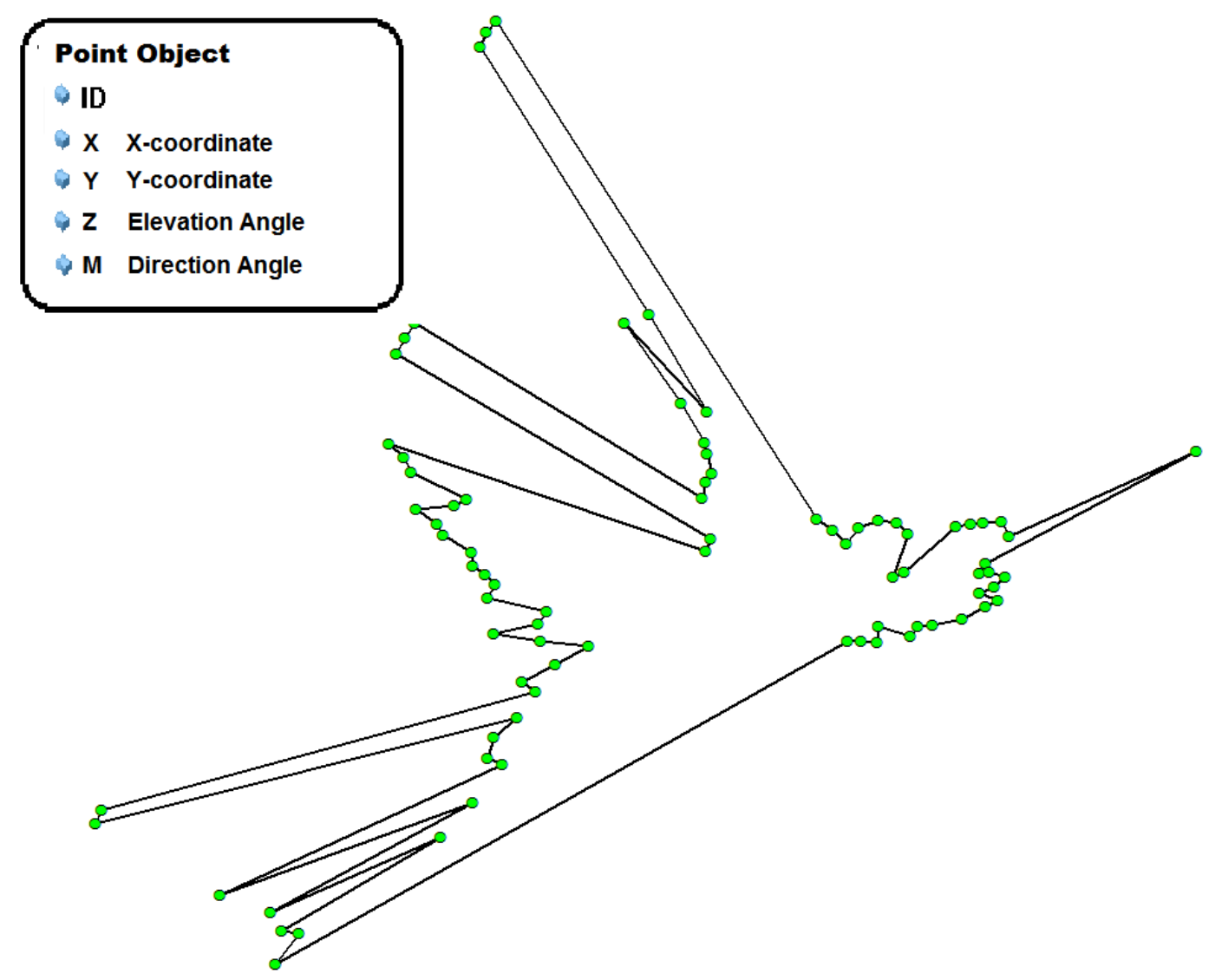

Figure 5-9: Horizon point objects drawn in XY space

Once the tool finishes running, the resultant dataset contains all the horizon lines, with each vertex on each horizon line storing all the variables necessary for the horizon analysis: X coordinate, Y coordinate, Direction Angle, Elevation Angle.

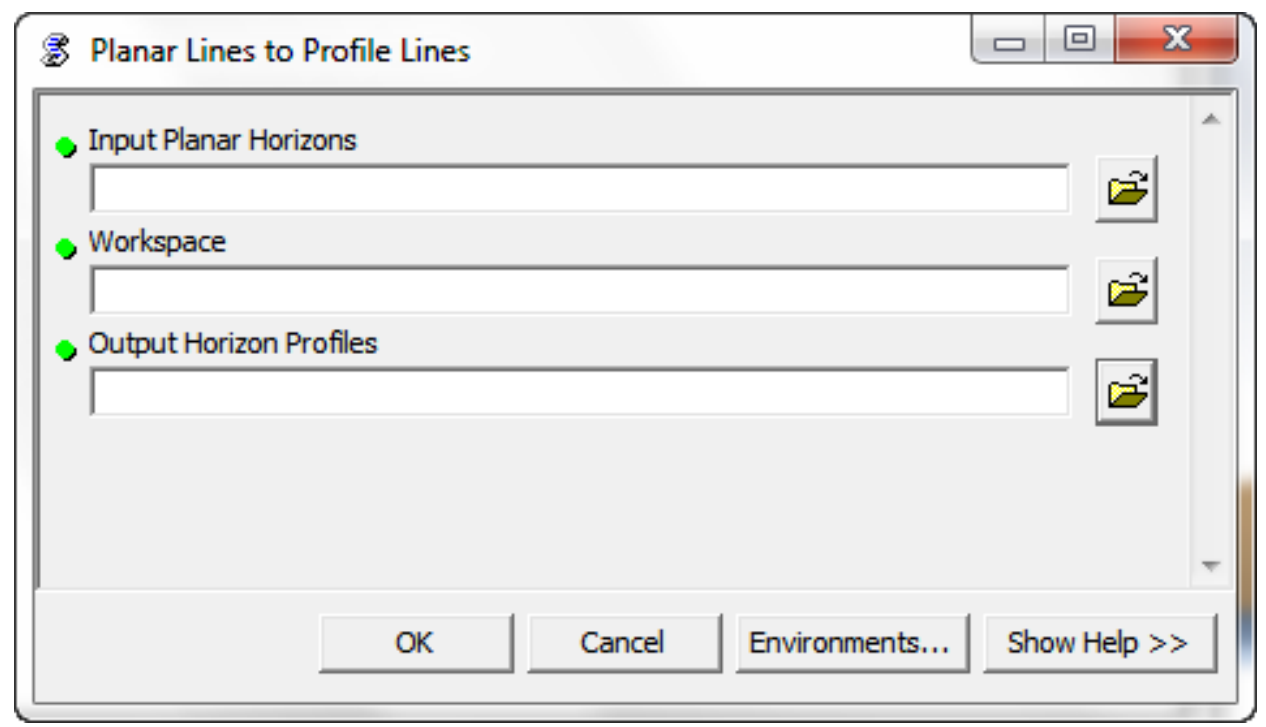

Figure 5-10: Planar Lines to Profile Lines tool 
The Planar Horizon Lines to Profile Lines Tool interface is shown in Figure 5-10. This tool takes a horizon line mapped on the XY plane and outputs a line feature class with the horizon mapped in profile. The script does this by accessing the geometry of each vertex and swapping the $\mathrm{X}$ attribute for the $\mathrm{M}$ attribute and the $\mathrm{Y}$ attribute for the $\mathrm{Z}$ attribute. Then the GUI draws the feature class, it draws the Direction Angle as the X coordinate and the Elevation Angle as the Y coordinate. Since the original X and Y coordinate values are stilled stored in the geometry object of the vertex, no information about the XY location of the original horizon line vertices is lost.
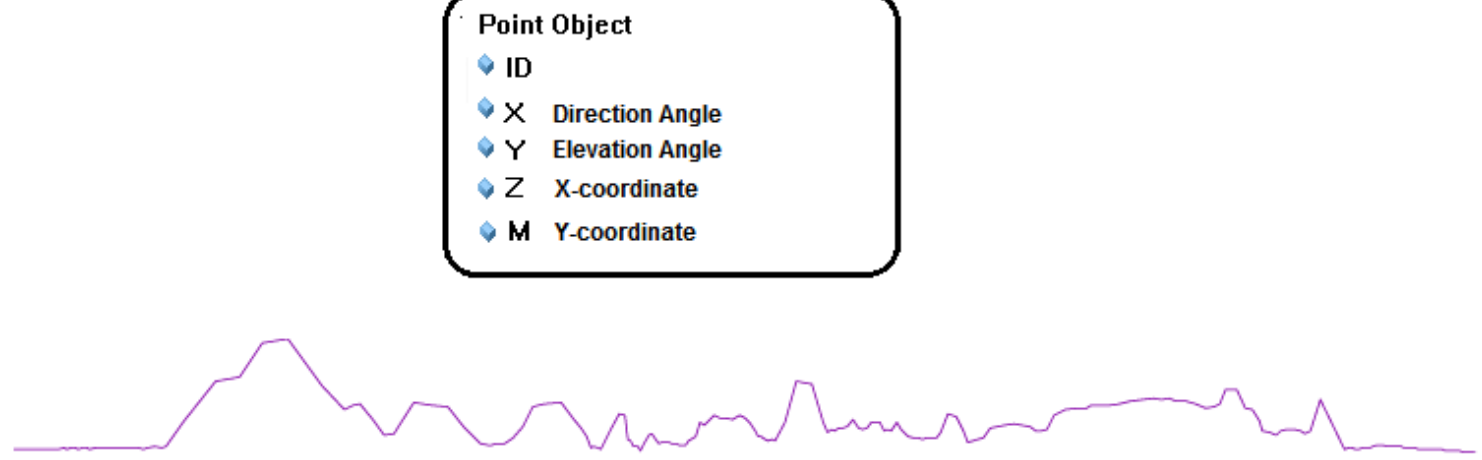

Figure 5-11: Horizon profile point object geometry

\subsection{Determining the Visual Significance of Horizon Peaks}

Section 2.3 of this report reviews the Douglas- Peucker algorithm and how it can be used to measure the visual significance of points along a line. The geoprocessing tools from Section 5.1 take planimetric horizon lines and map them in profile. The $\mathrm{X}$-axis represents the direction angle, from 0-360 degrees, between each vertex on the horizon line and the observer's location. The Y-axis represents the elevation angle between each vertex on the line and the observer.

The next goal after the horizon lines are mapped in profile is to calculate the visual significance of the peaks on each profile line. This is accomplished by running the Douglas- Peucker algorithm on a horizon profile line multiple times for a range of different simplification tolerances. Figure 5-12 demonstrates this process for a horizon profile line. 


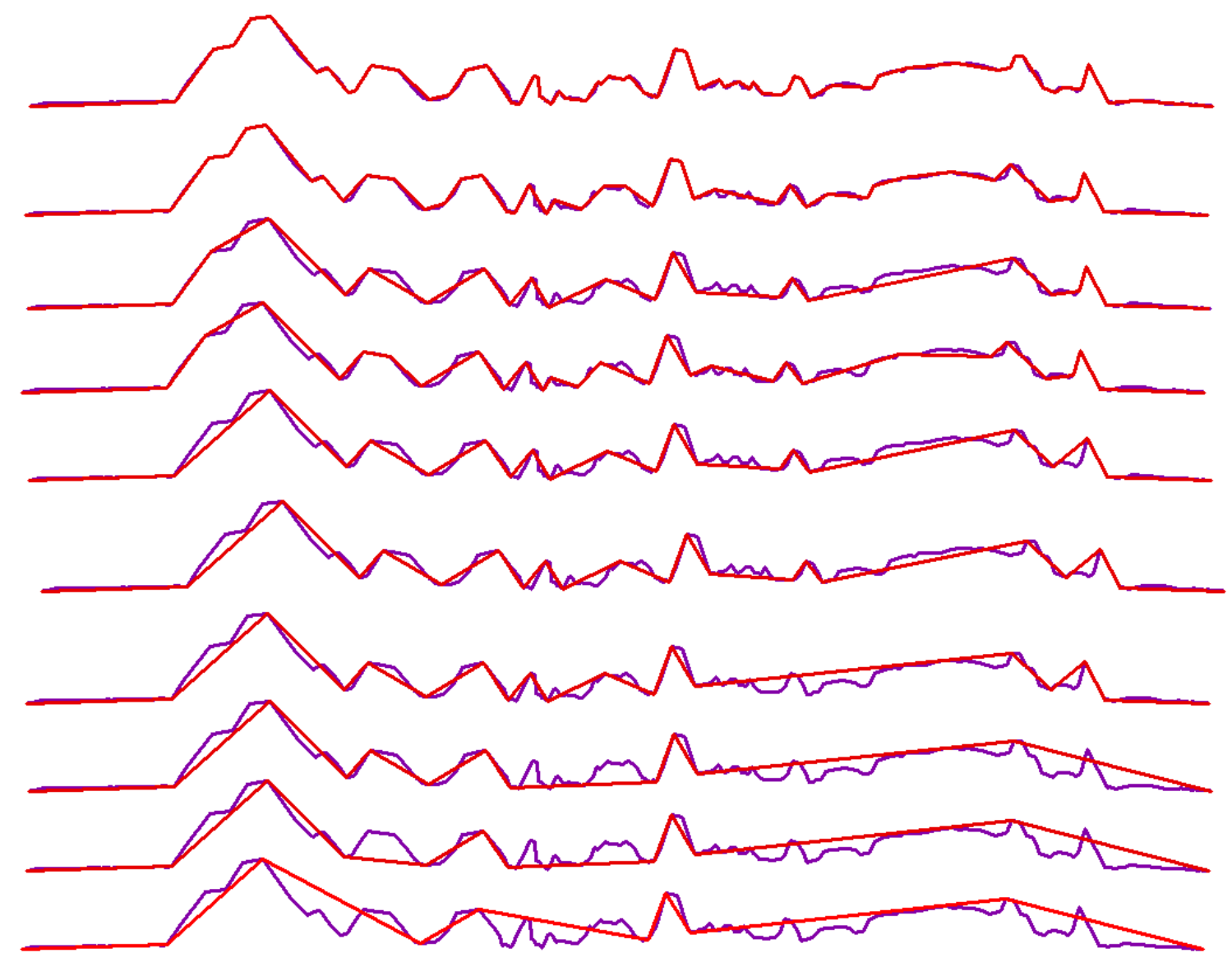

Figure 5-12: Using the Douglas- Peucker algorithm to select significant points

In this process, the algorithm runs ten times at ten different simplification tolerances. The simplification tolerance is a linear distance that specifies the maximum allowable offset between the original vertices and the final simplified line. The line generated by the algorithm at the top of the diagram shows the line resulting from a simplification tolerance of 1 map unit. In ArcMap, the map unit represents the unit of measure along the $\mathrm{X}$ and $\mathrm{Y}$ display axes. For the horizon profile, the $\mathrm{X}$ and $\mathrm{Y}$ axes both measure degree values. The separation between vertices in the $\mathrm{X}$ direction represents the directional angular separation between the two points with respect to the location of the observer (Figure 5-13). The separation in the Y direction represents the difference in elevation angles between the two points (Figure 5-14). In ArcMap, the Douglas-Peucker algorithm calculates the offset in map units between each vertex and the trend line using the Pythagorean theorem for distances between points on a plane and then compares this offset distance to the simplification tolerance. If the offset value is a greater number of map units than the simplification tolerance, the vertex is visually significant and included in the simplified line output. This line results in the least generalized line and chooses the most points as significant. In the horizon profile display, the map units represent degrees and the offset that ArcMap determines is a representation of the separation between points in angular space. For the first iteration, if the offset between a horizon profile vertex and the trend line is greater than 1 map unit, that point is considered significant to the shape of the horizon. The following iterations run the algorithm after increasing the 
simplification tolerance by 1 map unit from the previous iteration. The final iteration is the maximum simplification tolerance of 10 map units. This final output line chooses the fewest points as significant.

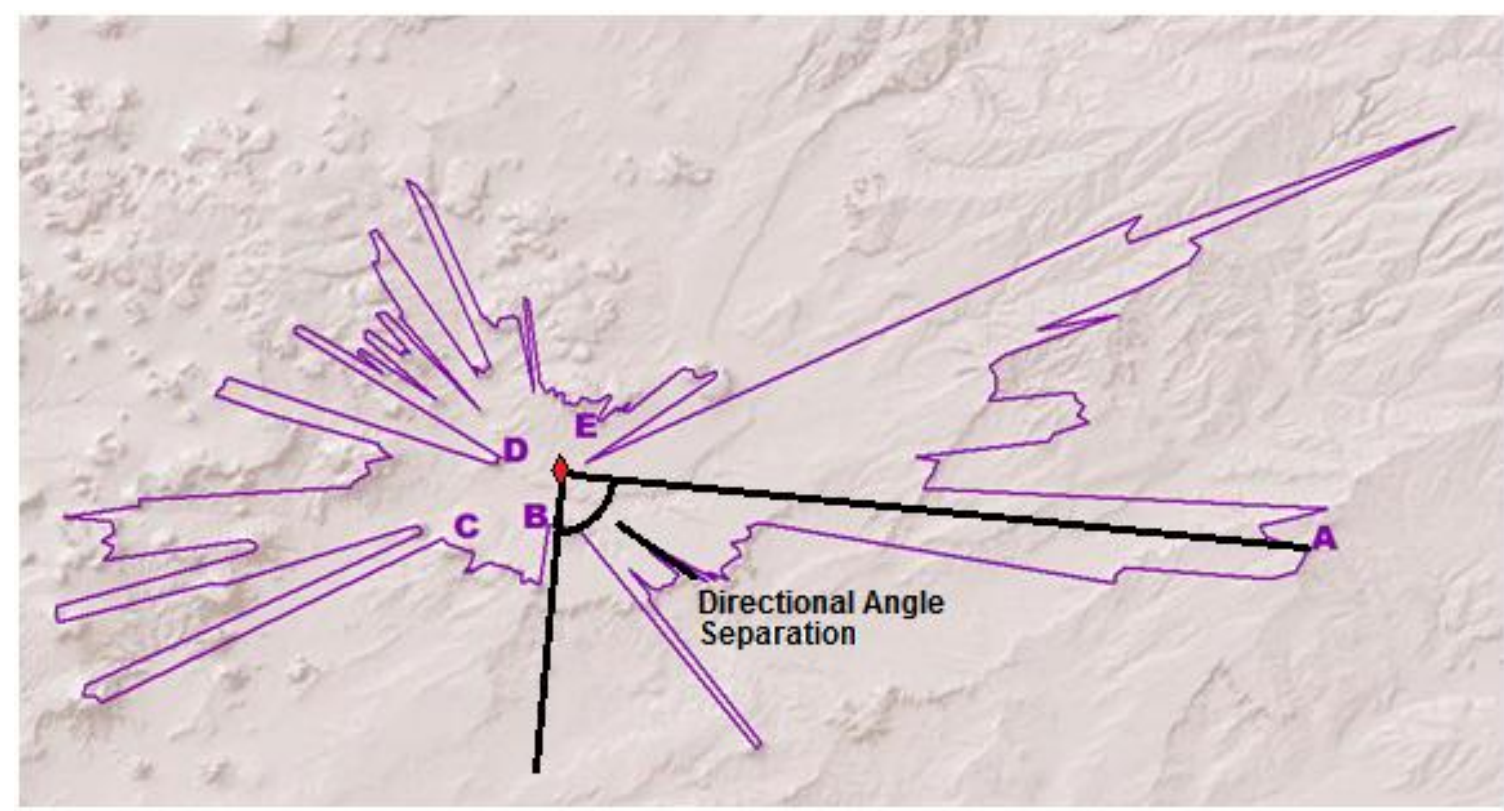

$\mathrm{X}$ separation $=$ Directional Angle Separation

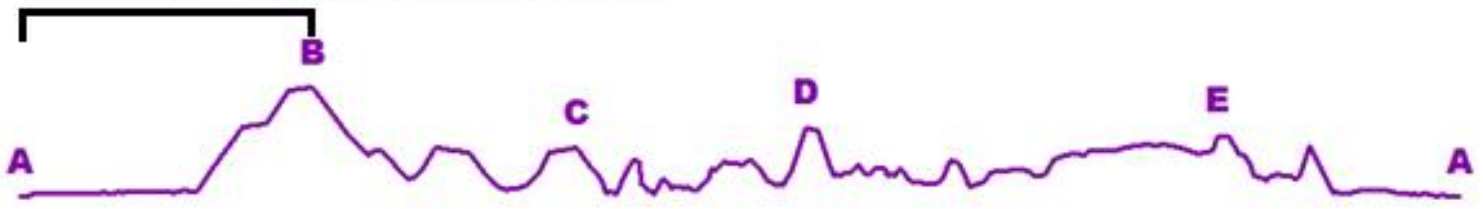

Figure 5-13: Directional Angle Separation in the planar view is equal to the $X$ Separation distance in the profile view. 

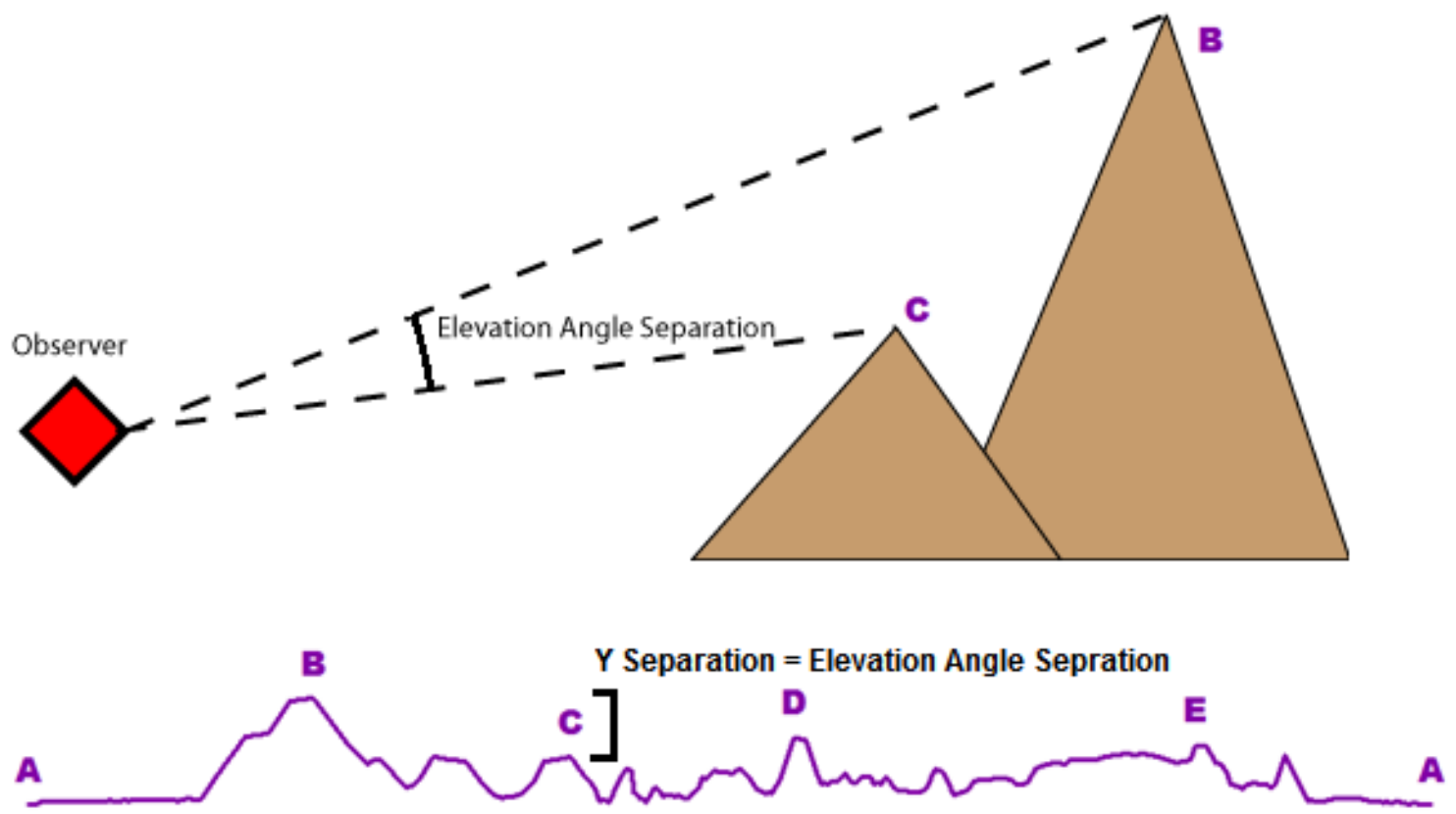

Figure 5-14: Elevation Angle Separation is equal to the Y Separation distance in the profile view.

Figure 5-15 shows the number of times each vertex was selected as significant by the Douglas-Peucker algorithm. The algorithm selected the most visually significant points all ten times the algorithm ran. The algorithm selected less visually significant points fewer times.

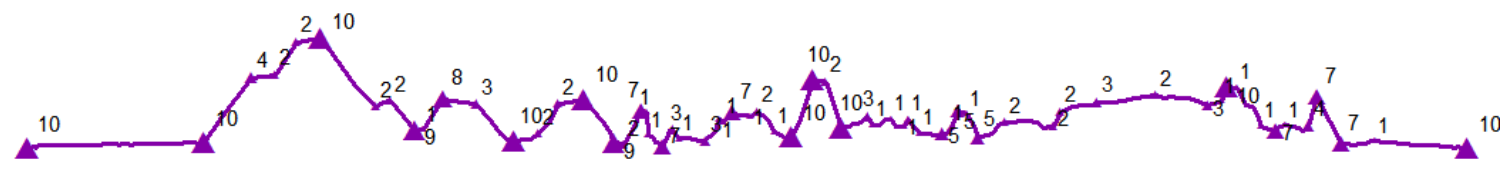

Figure 5-15: The final scores for all the vertices on one horizon profile.

The algorithm not only selects points that are local maxima, but also points that are local minima. When the final scores are counted, the local minima are discarded from the final peak dataset because the criteria for calculating visual significance states that peaks that appear taller have a greater visual significance. A vertex on the horizon profile line is considered a significant peak if it has a greater elevation angle than its two neighboring vertices. Figure 5-13 shows the horizon peaks for the example horizon profile. 


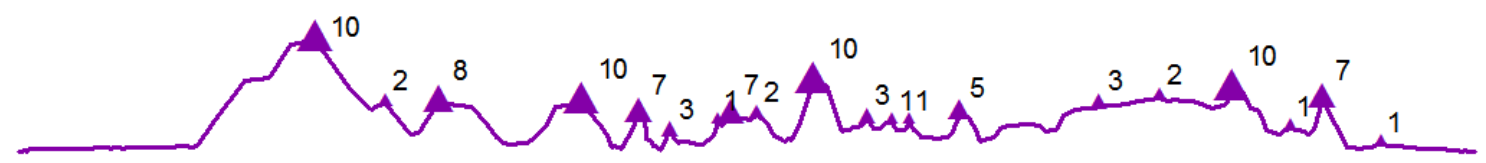

Figure 5-16: Horizon peaks and their visual significance count.

Another consideration that affects the results of the analysis is the fact that the Douglas-Peucker algorithm always begins each iteration by selecting the two endpoints of the line as significant. This means that whichever two points happened to have a direction angle of 0 on the horizon line will automatically be selected every time the algorithm runs and will therefore score the highest when the horizon points are counted, regardless of its relative visual significance. For this reason, instead of selecting North as the arbitrary 0 degrees on the Direction Angle axis, the point on the line with the lowest Elevation Angle is selected as 0 degrees and the direction angle of all other points on the line are rotated in the counterclockwise direction. This eliminates the arbitrary selection of the northernmost point as visually significant. The vertex with the lowest elevation angle will always be discarded from the output dataset of significant horizon peaks.

The direction angle ranges from 0-360 degrees along the $\mathrm{x}$-axis, whereas the range of the elevation angle tends to be much smaller. For this reason, when the lines are plotted they appear much flatter than in the real world and the algorithm tends to select only a few points even at very small simplification tolerances. In order to enhance the shape of the line, the elevation angle was vertically exaggerated. The result of this is that more points are selected as visually significant. The example in Figure 5-12 uses a vertical exaggeration factor of eight to display the horizon profile.

Figure 5-17 shows the user interface for running the "Get Simplified Lines" geoprocessing tool. This tool is used to run the Douglas- Peucker algorithm method for calculating visual significance of horizon peaks. 


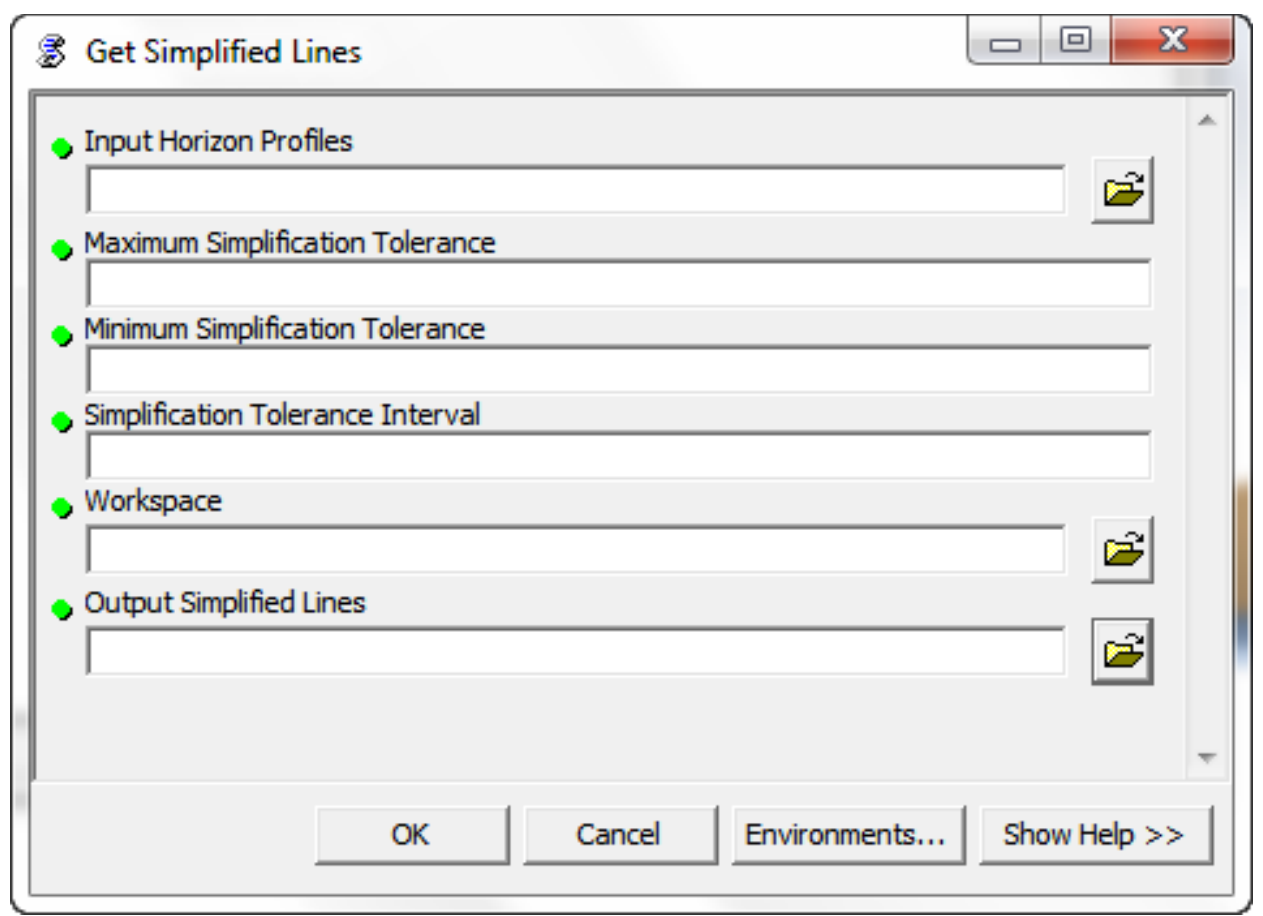

Figure 5-17: The "Get Simplified Lines Tool"

The "Get Simplified Lines" tool has six parameters. The first parameter is the input line dataset. This can be any line feature class. For this tool, the lines should be the horizon profile lines that are output by the "Planar Lines to Profile Lines" tool. The next three parameters are "Maximum Simplification Tolerance," "Minimum Simplification Tolerance," and "Simplification Tolerance Interval." These fields accept integer values. When the script runs, it takes the first line in the dataset and runs the Douglas-Peucker algorithm on this line with the minimum simplification tolerance. The line that is created from this output is stored in the "Output Simplified Lines" feature class. The script then increments the simplification tolerance by the simplification tolerance interval. The Douglas-Peucker algorithm runs on the first line again with the greater simplification tolerance. This process repeats until the simplification tolerance reaches the maximum simplification tolerance. Once the algorithm runs on the line with the maximum simplification tolerance, the iterations stop and the process repeats for the next line in the dataset. The final output is a feature class that contains all the simplified lines for each of the original horizon profile lines.

Figure 5-16 shows the interface for the "Count Significant Peaks" tool. This tool counts the number of times each point on the horizon line is present in a simplified version of its horizon profile line. 


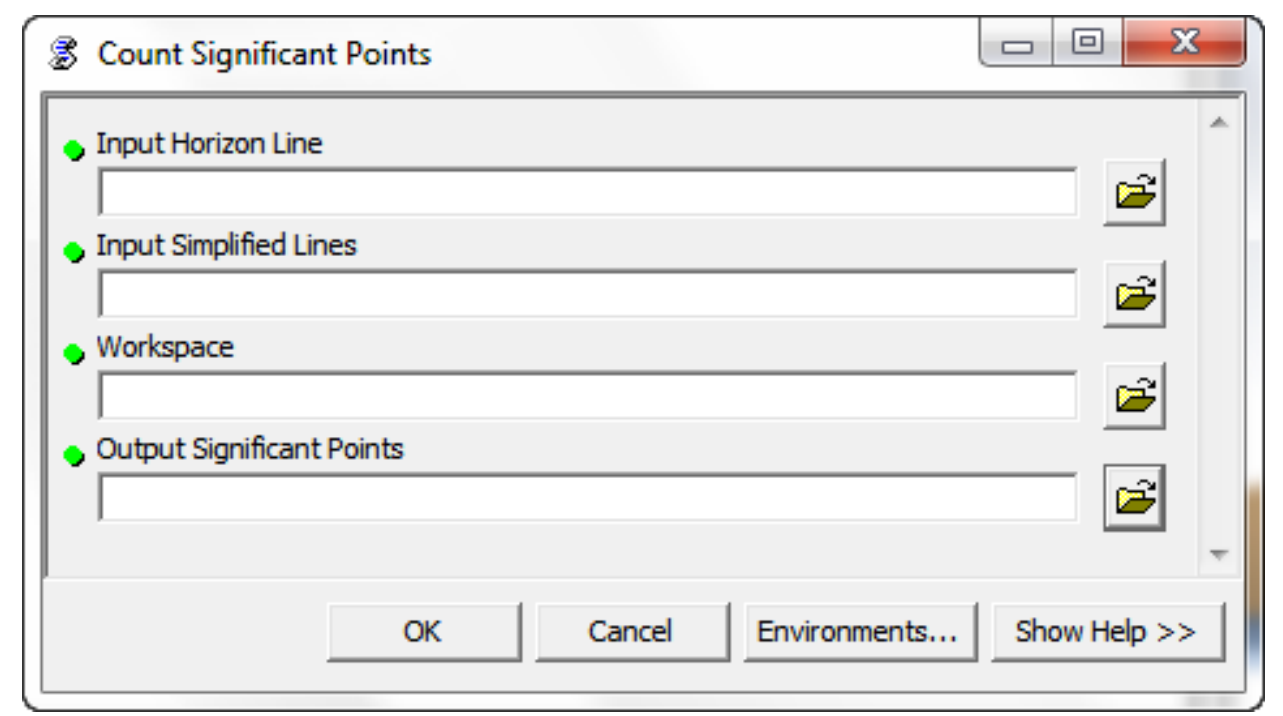

Figure 5-18: The "Count Significant Points" field

This tool takes an input horizon line dataset and a set of simplified lines related to that dataset via the "SiteID" field. The script then tallies the number of times each vertex in the original horizon line is present in the simplified line dataset. The more times a vertex is counted, the more times it was selected as visually significant to the shape of the horizon line. The output feature is a point dataset that includes a field called "Count." This field is an integer value that indicates the number of times each vertex was selected as visually significant.

\subsection{Mapping Horizon Peaks in the XY plane}

The dataset of horizon points are mapped in the Distance Angle vs. Elevation Angle coordinate frame. To view the significant peaks in the XY plane from the cartographic perspective, the "Planar Lines to Profile Lines" reads the X and Y coordinates that are stored in the geometry of every point object, and writes these to a new point feature class in which the $\mathrm{X}$ and $\mathrm{Y}$ coordinates are stored in the $\mathrm{X}$ attribute and $\mathrm{Y}$ attribute of the point geometry, respectively. Figure 5-17 shows the user interface of the "Planar Lines to Profile Lines" tool. This tool accepts a point dataset of horizon points as input. The "Workspace" field is the geodatabase that stores the input and output dataset. The "Output Points" stores all the vertices of the horizon profile. The "Output Peaks" dataset stores only vertices that are local maxima and therefore classified as peaks. The client requested that both of these datasets be saved, not just the peaks dataset. 


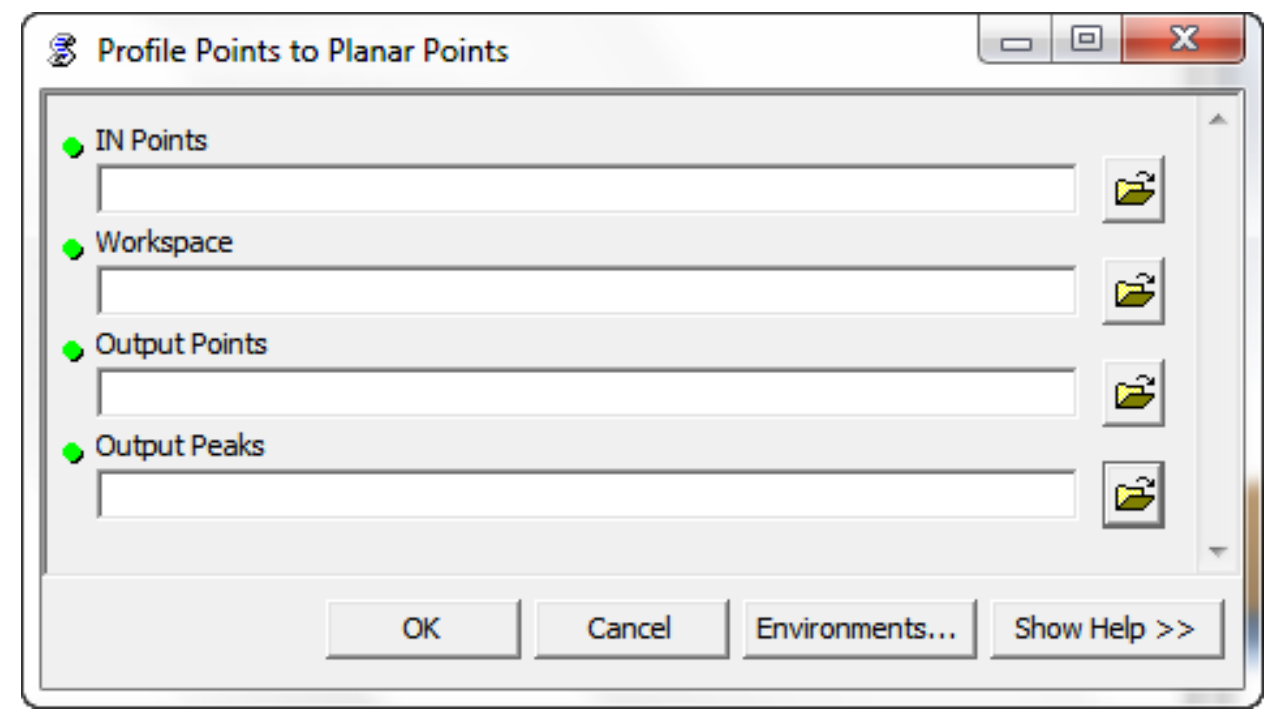

Figure 5-19: The "Planar Lines to Profile Lines" tool

Figure 5-20, Figure 5-21, and Figure 5-22 show the final results of the entire process of calculating the visual significance of horizon peaks. The triangles in each map represent the peaks and the number label next to it represents the final count of visual significance. The horizon profile is also shown above the planimetric view for comparison. 

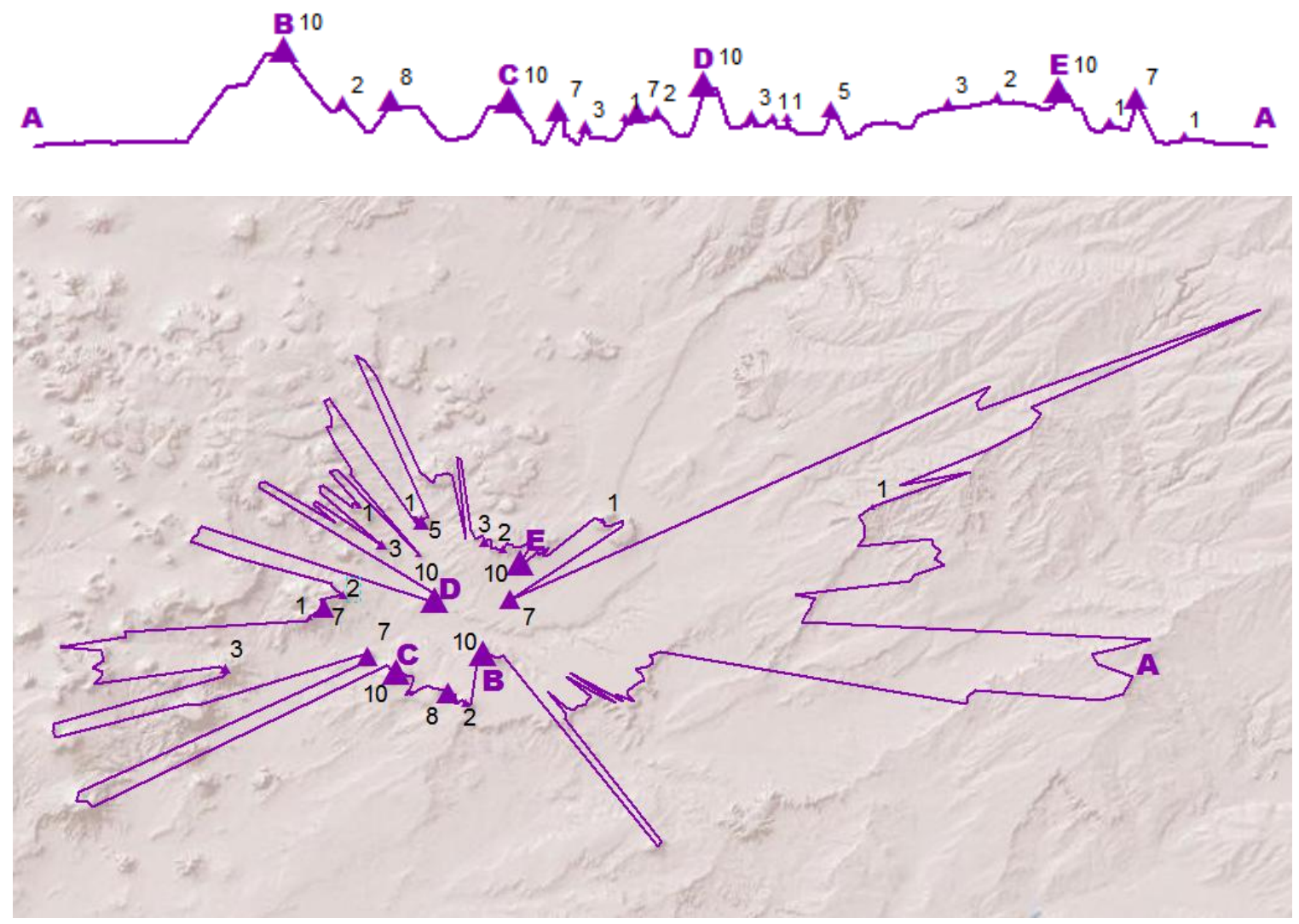

Figure 5-20: Example result of planimetric horizon peaks and their visual significance count 


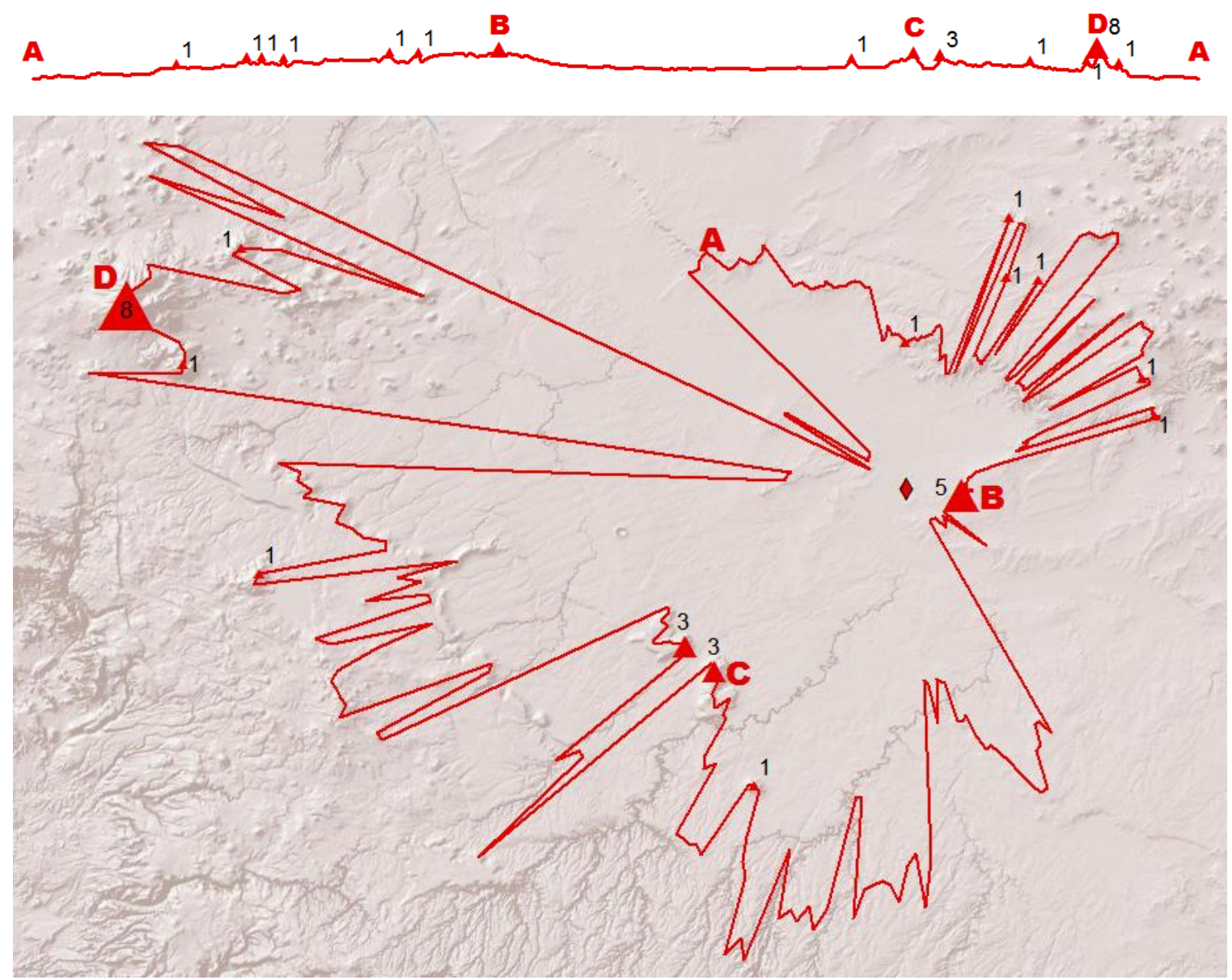

Figure 5-21: Example result of planimetric horizon peaks and their visual significance count 

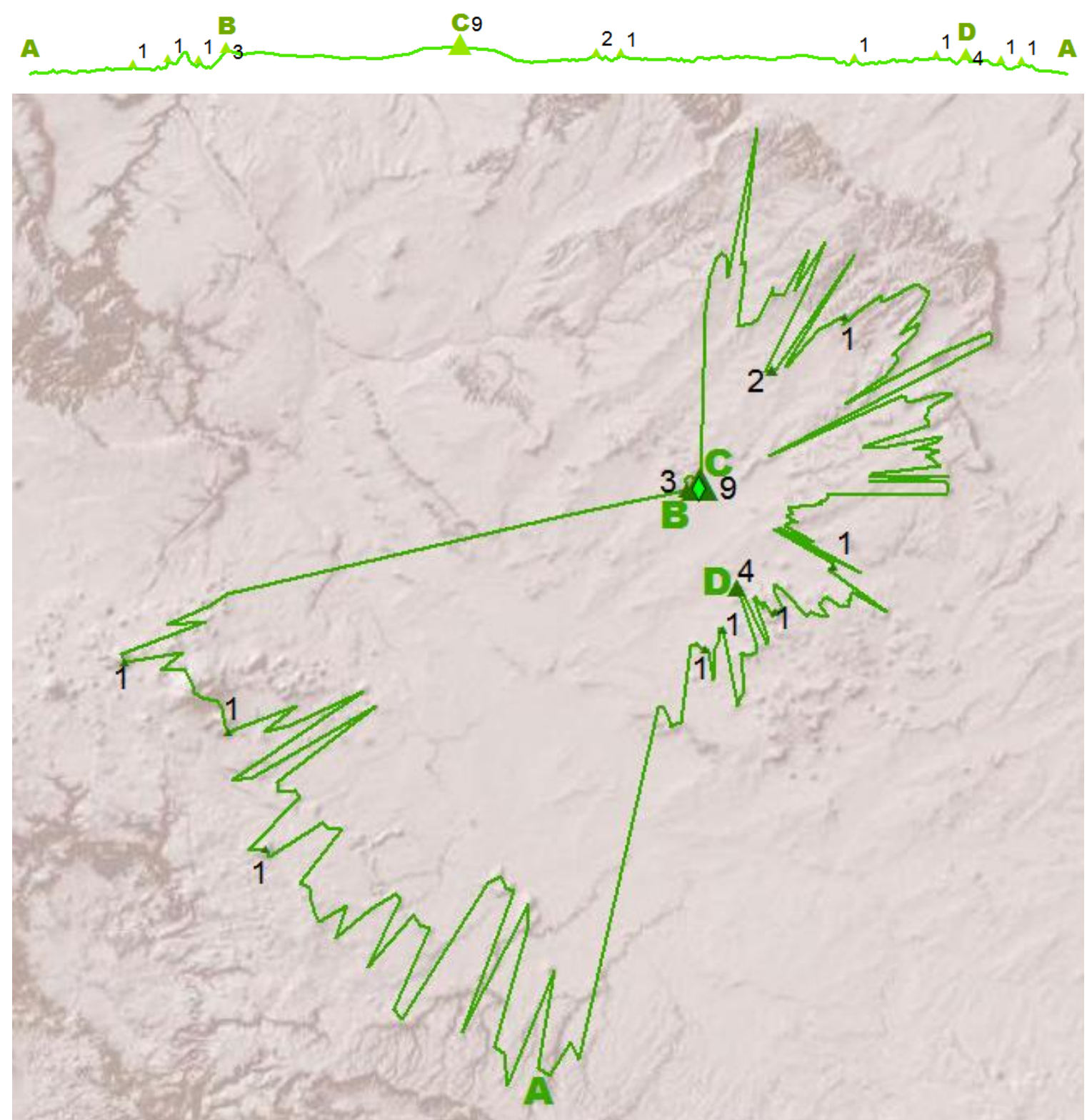

\section{Figure 5-22: Example result of planimetric horizon peaks and their visual significance count}

The three example profiles demonstrate the variety of shapes that a horizon profile can take. In the example shown in Figure 5-20, there is a greater difference in elevation angle among peaks along the line. The result is that more points received higher visual significance than in the other two examples. In the second example, the changes between elevation angles along the horizon profile are much less drastic. The result of this was that none of the peaks was selected by the algorithm for all ten runs. The most significant peak received a score of 8 . In the third example, the highest scoring peak received a score of 9. This peak also happens to be the closest peak to the observer location. 


\subsection{Aggregating Visual Significance scores to USGS Standard Peaks}

Each village has a unique horizon line. Each horizon line has its own array of point objects that represent the location of peaks on the landscape. Many horizon lines include the same peak, but because of the different perspectives of that peak and the limited precision of the coordinates, in the dataset horizon peaks that refer to the same real world peak may not have precisely the same coordinate values. Each point in the Horizon Peaks $\mathrm{XY}$ dataset is unique to a specific Hopi village. Many of these peaks are actually the same topographic feature but with slightly different coordinate value. This makes it difficult to total the visual significance scores that a single peak received from multiple villages. The next step in the implementation was to determine which peaks in the horizon peaks dataset represent the same physical feature and then calculate the total sum of the visual significance count for each peak in the USGS peaks dataset. In this step, the client specified a value of 400 meters as the search radius. Any horizon peak that was within 400 meters of its closest USGS peak would be considered the same as that peak. The ArcGIS Toolbox "Near" tool was used to find the distance between each of the Horizon Line peaks and the Standard USGS Peak dataset provided by the client. If a Horizon Peak was not within 400 meters of a Standard Peak, then that peak was not considered the same peak any of the USGS peaks.

Figure 5-23 illustrates how the scores in the Count field for each Horizon Peak were summed to the USGS Peak dataset. The gray circles represent 400 meter buffers around the standard peaks. The larger black triangles represent USGS peaks. The smaller triangles represent a horizon peak. Each color signifies the different villages each horizon peak is associated with, and the label indicates the visual significance score that horizon peak received along the horizon of that village. The attribute field "Count" and "siteCount" are added to the USGS Peaks attribute set. The Count of every peak that is within a Standard Peak's 400 meter buffer is added to the Count field. Peaks that are within two buffers contribute their visual significance count to the Count field of the Standard Peak to which they are nearest. 


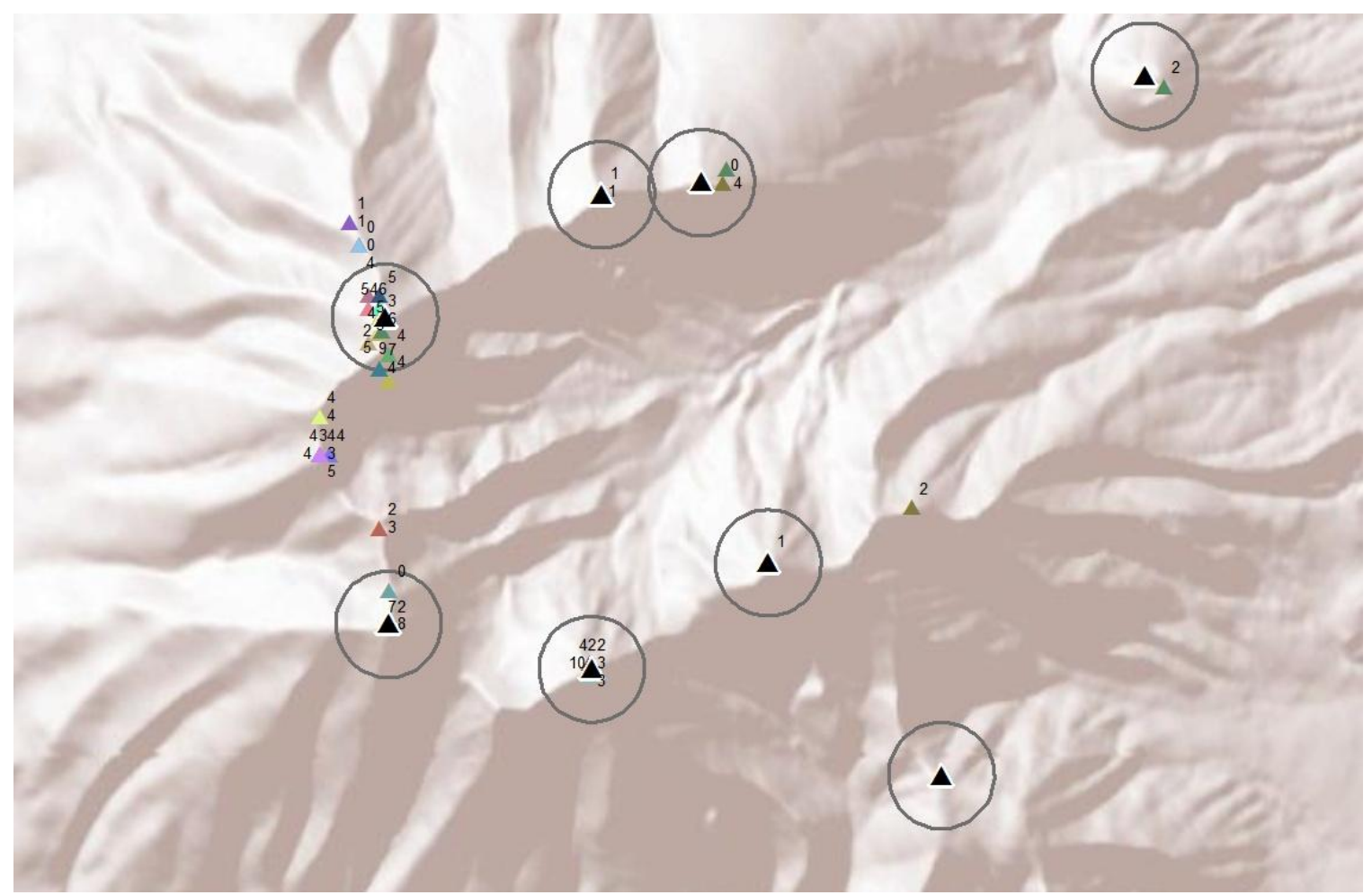

Figure 5-23: 400 meter buffers around USGS Peaks (black triangles). Horizon Peaks labels indicate the visual significance score that peak received with respect to the village that observed it.

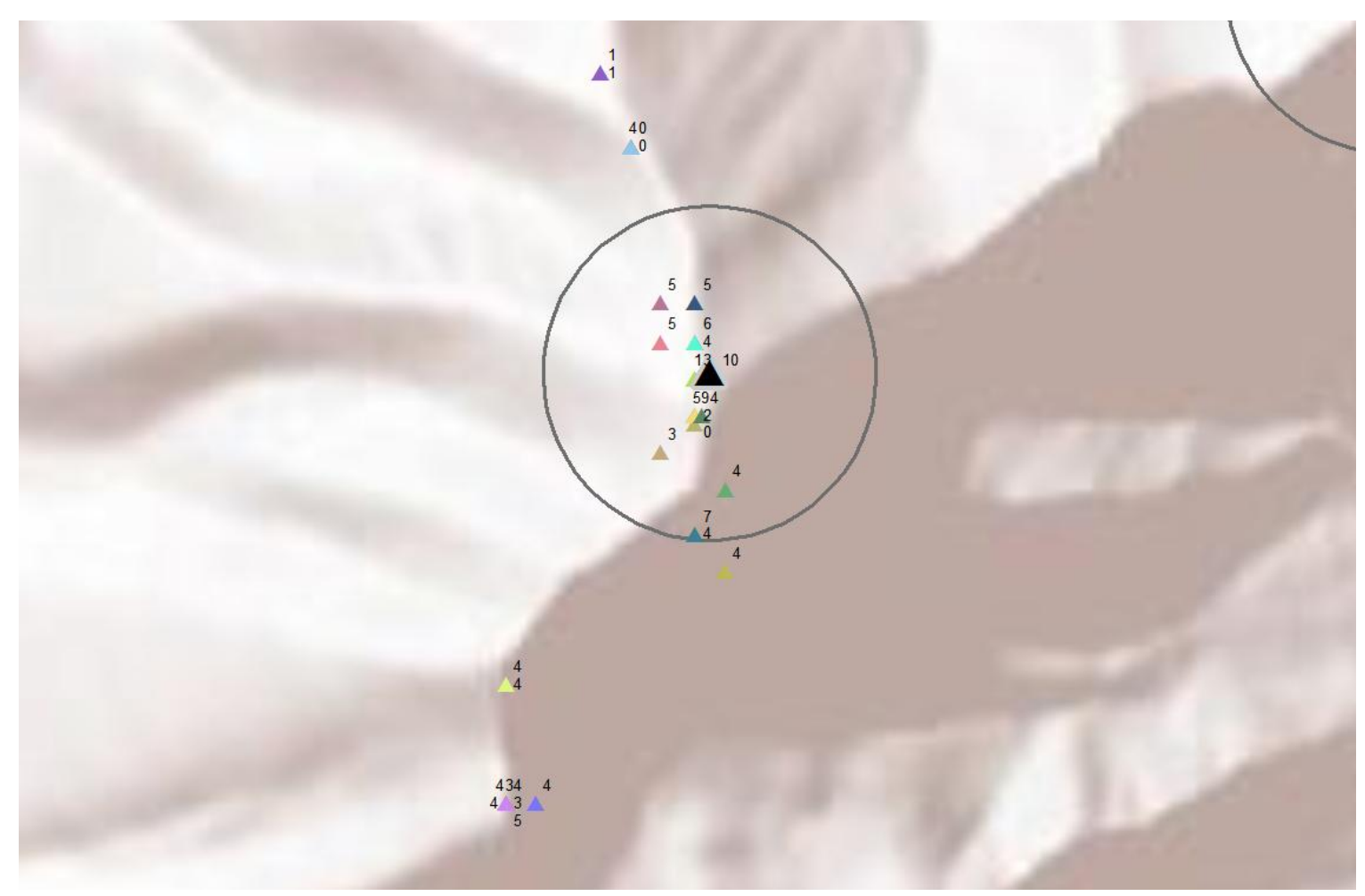

Figure 5-24: Horizon Peaks within 400 meters of a USGS peak 
The final result of the aggregation process is a total visual significance score for each of the peaks in the client's USGS Peaks dataset. The siteCount field is the number of peaks that had that Standard peak on their horizon line. A peak may have a very high Count and a high siteCount; this means it was partially significant to many peaks. Alternatively, if a peak has a high Count but a low siteCount, it likely had high visual significance scores for only a few villages.

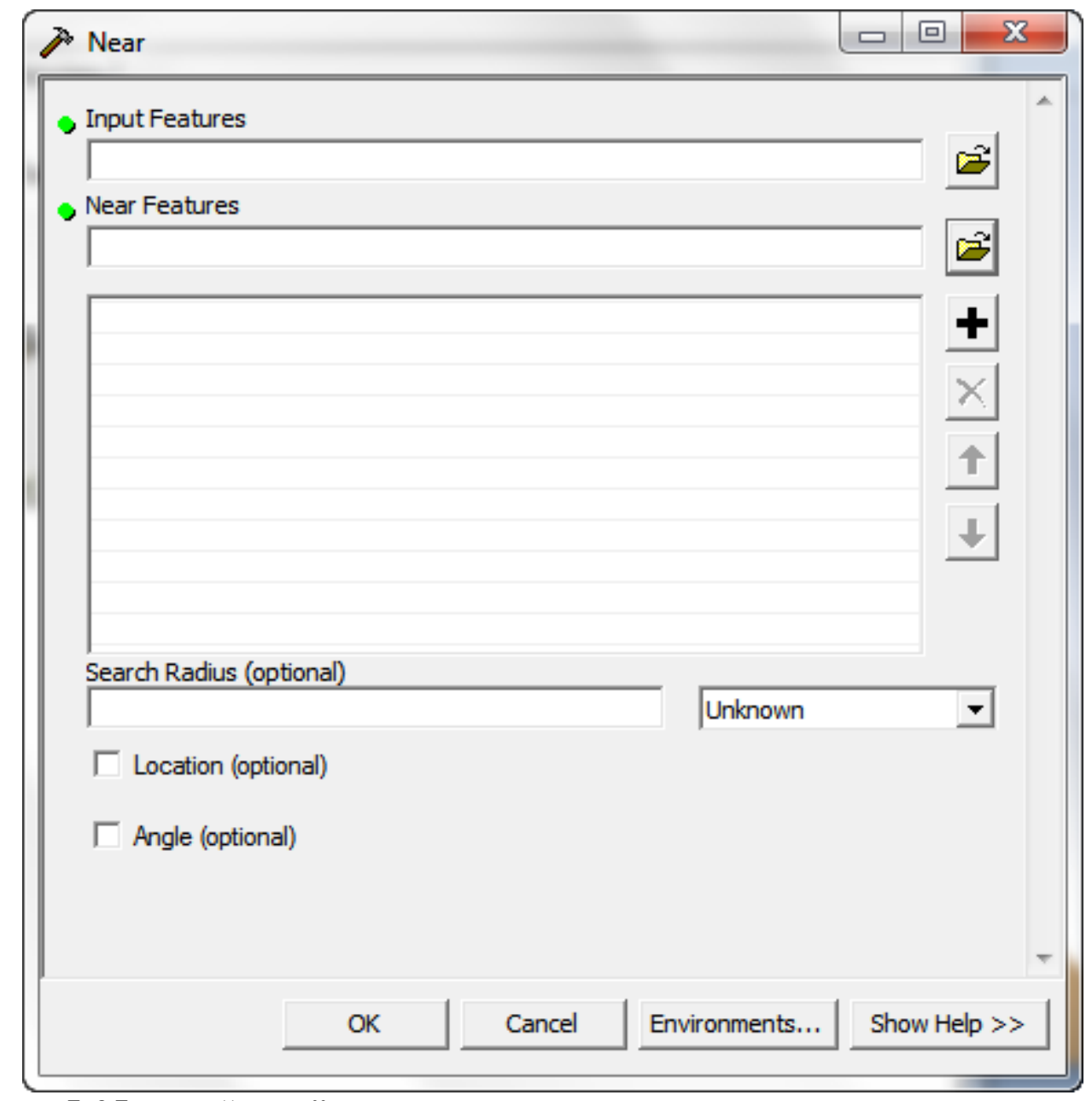

Figure 5-25: The "Near" tool

The Near tool was used to find the closest Standard Peak to every Horizon Peak. The Input Features are the Horizon Peaks. The Near Features are the Standard Peaks. The Search Radius used was 400 meters, as specified by the client. The Near tool adds a NEAR_FID attribute field and a NEAR_DIST field to the Horizon Peaks Table. The NEAR_FID field is the FID of the Standard Peak that each Horizon Peak is closest to, if they are within 400 meters. The NEAR_DIST field is the distance between a Horizon Peak and its nearest Standard Peak, if the distance if under 400 meters. If a Horizon Peak is not within 400 meters of a Standard Peak, it will have a value of -1 in the NEAR_FID and NEAR_DIST field. 


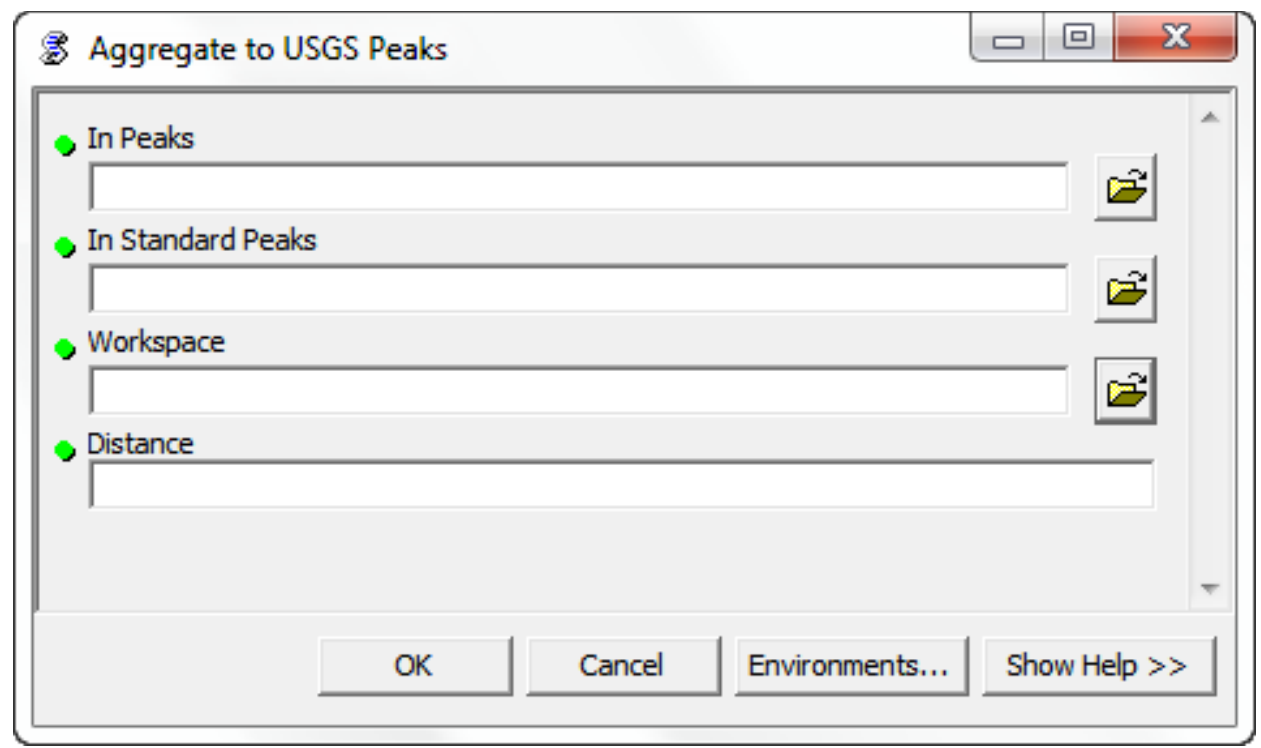

Figure 5-26: The "Aggregate to Standard Peaks" tool

The "Aggregate to USGS Peaks" tool is a custom geoprocessing script. This tool takes the Horizon Peaks as Input Peaks and the Standard Peaks in the In Standard Peaks field. This tool creates a "Count" attribute field and a "siteCount" attribute field in the Standard Peaks table. The tool takes each USGS peak and finds all Horizon Peaks that are nearest to that peak and within the specified distance and stores the number of peaks found in the siteCount field. The tool then sums all the values in the Count field of those Horizon Peaks and adds the total to the Count field of the USGS Peaks. 


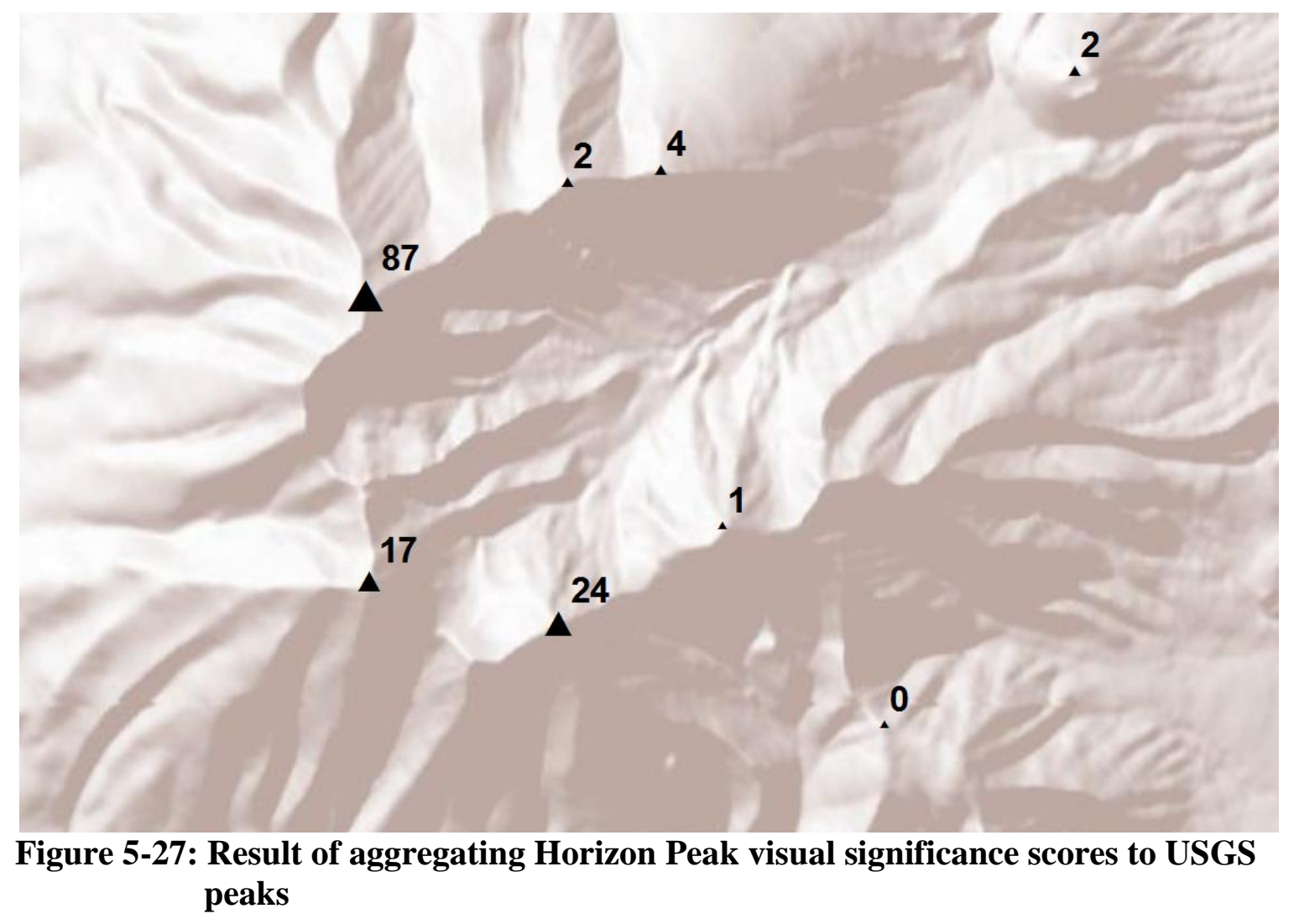

Figure 5-27 shows the results of aggregating Horizon Peak count scores to USGS peaks for eight of the peaks in the dataset. Figure 5-28 shows a map of the USGS peaks for the entire study area after all the Horizon Peaks scores were aggregated. The USGS peak symbol size is proportional to its visual significance count total. The larger the symbol size, the greater total visual significance it had during the time period of interest (1150- 1700 c.e.) 


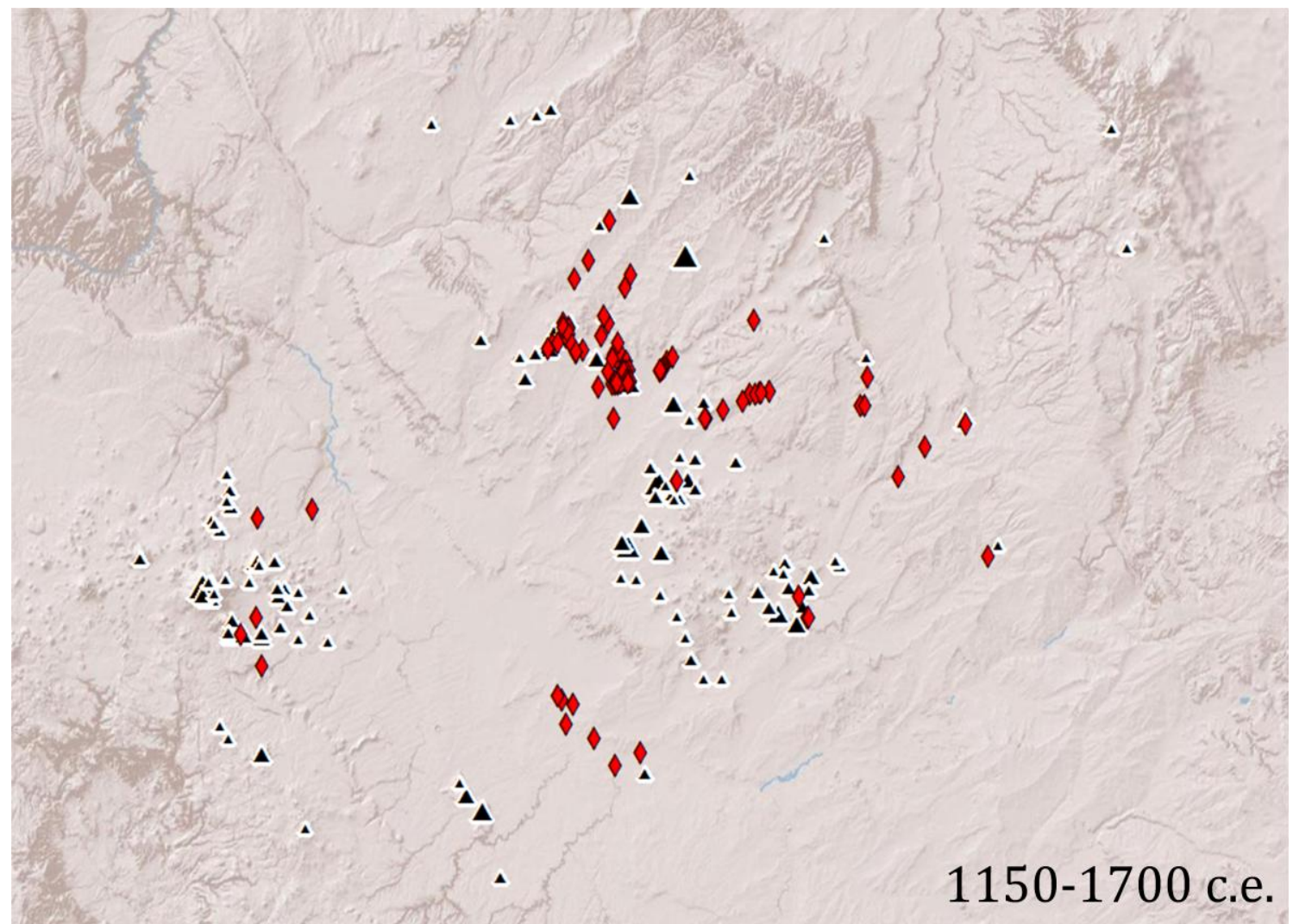

Figure 5-28: Total visual significance of USGS peaks

\subsection{Representing the change in visual significance over time}

The final functional requirement of the horizon analysis was to map how the visual significance of Peaks changed over time as the Hopi migrated to different villages. The client provided time data for every village between the years $1150-1700$ c.e. The time data are represented in 50 year intervals between 1150-1700 c.e. If a village was in existence during a fifty year interval it has a 1 in that field; if it was not in existence it has a 0 value. For example, the village in Table 5-28 has a 1 in the _1349, 1399 , and _1449 fields, and a 0 in all the other fields. This indicates that the village was in existence from the 1300-1349 interval, the 1350-1399 interval, and the 1400-1449 interval, but was not inhabited during any of the other intervals

\begin{tabular}{|r|r|r|r|r|r|r|r|r|r|r|r}
\hline 1149 & $\_1199$ & $\_1249$ & $\_1299$ & $\_1349$ & 1399 & 1449 & 1499 & 1549 & _1599 & $\_1649$ & $\_1699$ \\
\hline 0 & 0 & 0 & 0 & 1 & 1 & 1 & 0 & 0 & 0 & 0 & 0
\end{tabular}

\section{Figure 5-29: Time interval attribute data for a Hopi village}

To map change in time, the first step is to create a layer of extant villages in the ArcMap display for each time interval. For the 1100-1149 time interval, the query "_1149" >0 is used to select all villages that have a 1 in the _1149 field and were 
therefore inhabited during this time period. Next, the "Create Layer from Selected Features" option is selected and the layer is named "Sites_1149." Next all Horizon Peaks that have a SiteID that matches a SiteID in the Sites_1149 layer are selected and a HorizonPeaks_1149 layer is created. Next, a copy of the Standard Peaks Layer is created called "Standard Peaks_1149." The "Aggregate to Standard Peaks" tool is run, with the HorizonPeaks_1149 and StandardPeaks_1149 layers as input. The result layer has a Count and siteCount field that has the total Count and siteCount fields for all the Standard Peaks, using only Horizon Peaks for villages inhabited during the $1100-1149$ time interval.

This process is repeated for each interval and the result is a map symbolizing the aggregate visual significance of the peaks based on villages during a time interval. These maps are 50 year snapshots that reveal changes in the visual significance of peaks over time based on where Hopi villages were located during that 50 year interval. Some peaks increase in Aggregate Visual Significance over the years, other Peaks decrease. Some peaks have no visual significance for villages during one time period, but do for another time period. Figures 5-29, 5-30, and 5-31 show the total visual significance of USGS peaks for three different fifty year intervals.

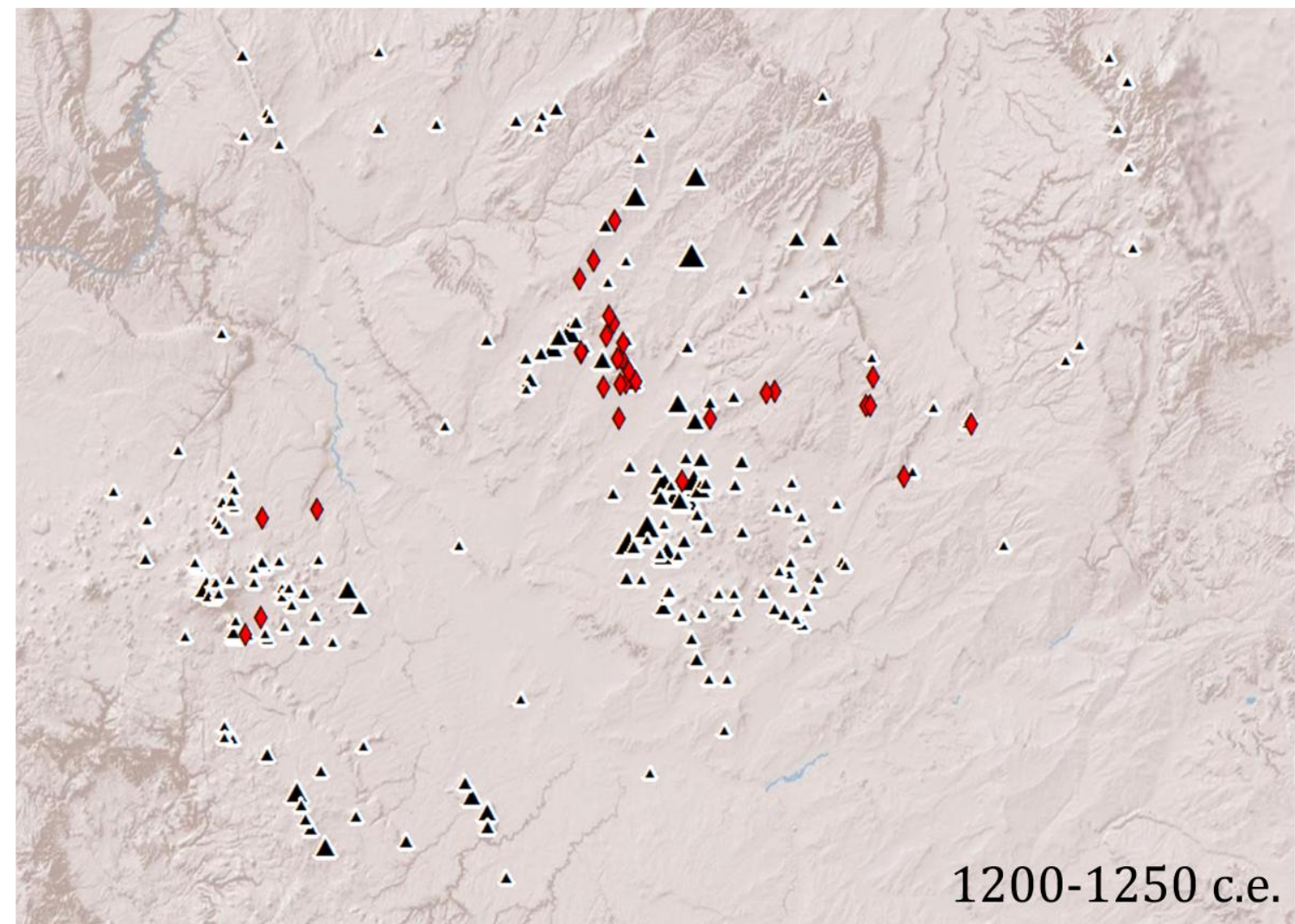

Figure 5-30: Total visual significance of peaks from 1200-1250 c.e.

Figure 5-30 shows a map of the villages in existence from $1200-1250$ c.e. The USGS peaks are symbolized as black triangles with their size proportional to their total visual significance. 


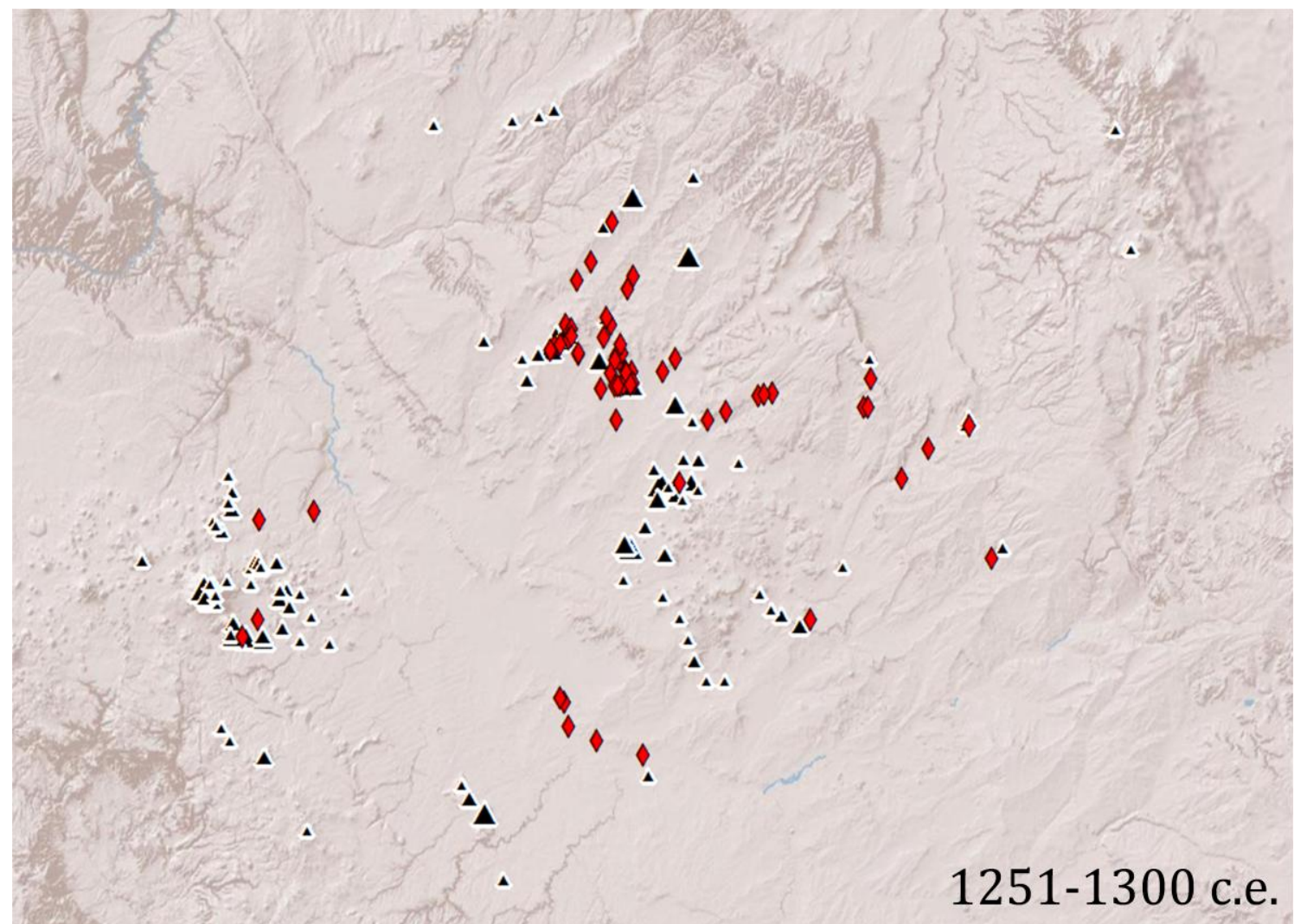

Figure 5-30: Total visual significance of peaks from 1251-1300 c.e.

Figure 5-30 shows the total visual significance of peaks from the 1251- 1300 interval. There are some differences in the total visual significance during this time period and the 1200-1250 time period. For instance, in the northeastern area of the map, there are seven visually significant peaks for the 1200-1250 time period, but there are only two visually significant peaks in this are for the 1250-1300 time period. 


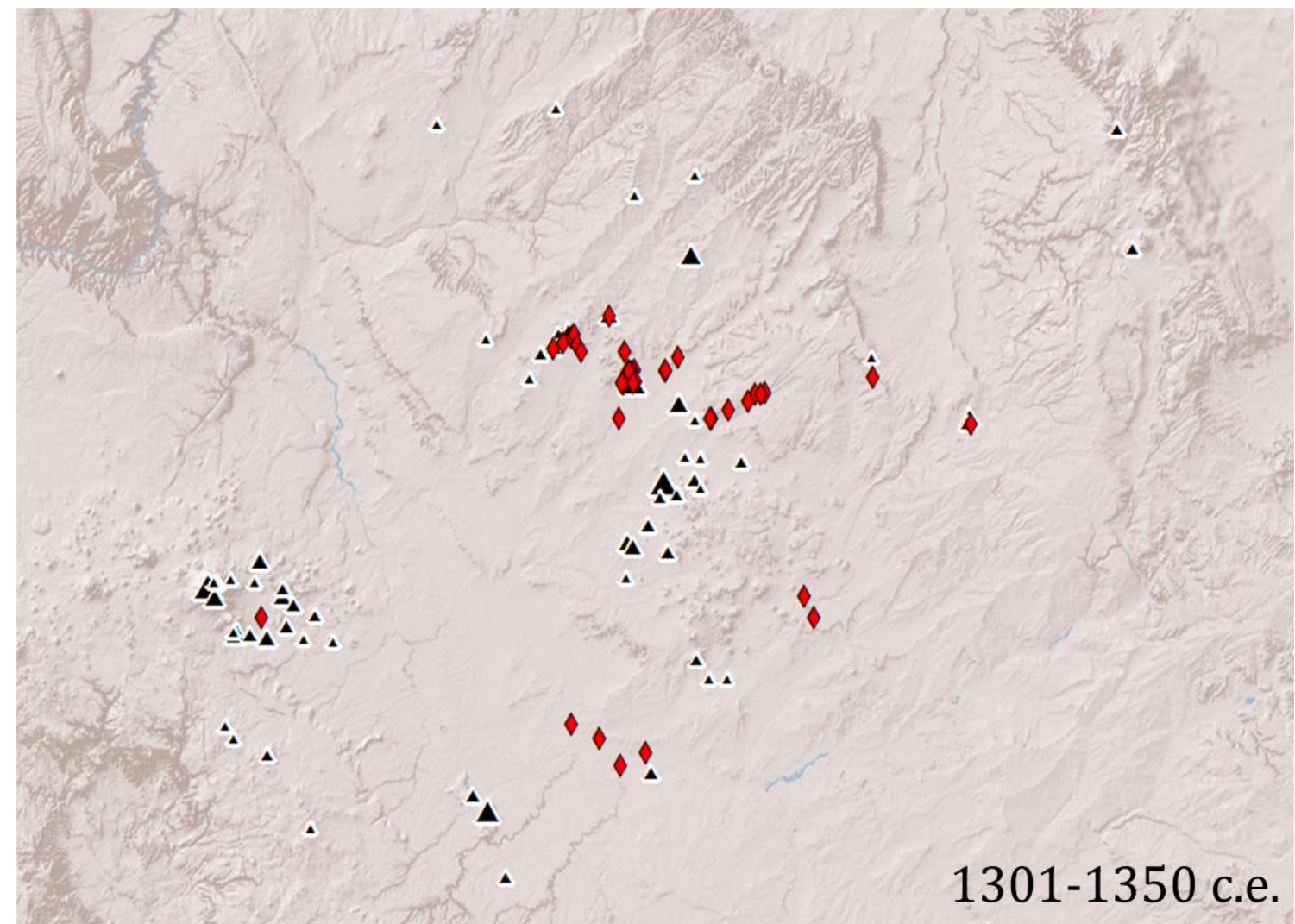

Figure 5-31: Total visual significance of peaks from 1301-1350 c.e.

The map for the 1301-1350 time period is shown in Figure 5-31. This map has fewer visually significant peaks in the mountain range in the southwestern region. This is likely explained by the abandonment of three villages in that region during this time period.

\subsection{Summary}

The implementation of the Hopi horizon analysis involved the development of several geoprocessing tools. When operated in sequence, the geoprocessing tools comprise a workflow that begins with a set of Hopi villages and horizons and ends with a series of maps that reveal changes in the visual significance of peaks over time.

The first geoprocessing task is to map the planimetric horizon lines in profile. The "Calculate Direction and Elevation Angles" tool calculates the direction angle and elevation angle for every vertex on a horizon line relative to the location of its corresponding village. These angles are stored in the Shape field of the output dataset.

The next tool in the workflow is the "Planar Lines to Profile Lines" tool. This tool takes the planimetric horizon lines and outputs a dataset that displays the horizon line in profile, with the direction angle on the $\mathrm{X}$-axis of the map display and the elevation angle on the Y-axis.

The next step in the workflow is the calculation of the visual significance of horizon peaks. This is accomplished by running the Douglas-Peucker line simplification algorithm on each horizon line at ten different tolerance levels. The next tool, "Count Significant Points," counts the number of time the algorithm chose each vertex on the 
horizon line and stores the total for each point in a point feature class. The "Profile Points to Planar Points" tool converts the horizon peaks from profile view to planimetric view.

Now that the horizon peaks display in planimetric space, the Near tool is used to determine which USGS peak is the nearest to each horizon peak. All horizon peaks that are within 400 meters of a USGS peak are considered the same peak for the analysis. The "Aggregate to USGS Peaks" tool calculates the sum of the visual significance count for each horizon point within a USGS peak's 400 meter buffer zone and adds this total to the attribute field.

After the completion of the geoprocessing workflow, the final step of the analysis is the visualization of the resultant data. This is accomplished by finding the total visual significance of USGS peaks for villages in existence during fifty year intervals from $1100-1700$ c.e. The final product is a series of seven maps, with each map corresponding to a 50 year interval. 



\section{Chapter 6 - Results and Analysis}

The Hopi Horizon Analysis project had four primary objectives. The first objective was to determine a method for quantifying the visual significance of horizon peaks. The second objective was to build a geodatabase that stored the project data and supported the analysis. The third objective was to develop geoprocessing tools that would perform the horizon analysis on the data in the geodatabase. The fourth objective was to produce visualization of the analysis results that reveal the change in visual significance of the peaks over time.

The first objective of the horizon analysis project was to determine a method for quantifying the visual significance of peaks along a horizon that was dependent on the location of the horizon observer. Several methods were considered, but ultimately the client agreed to use an approach using the Douglas- Peucker algorithm. This method involved four steps: 1) Map the planimetric horizon lines in profile, 2) run the DouglasPeucker algorithm on each line at ten different levels of significance, 3) count the number of times the algorithm selected each point as visually significant, and 4) map the horizon peaks in the planimetric perspective.

Mapping the planimetric horizon lines in profile was successful. Using the built in geometry that is part of the ArcGIS geodatabase, it was possible to display the line data in profile, with the $\mathrm{x}$-axis representing the direction angle between each vertex and the observer location and the $y$-axis representing the elevation angle. One problem with mapping the horizon lines in profile was that in many cases, the elevation angles were much smaller in scale than the direction angle, which ranges from 0-360 for all the lines. The result of this was that many lines appeared flattened. The solution to this was to use a vertical exaggeration factor to compensate for the discrepancy in scales between the $\mathrm{x}$ axis and the y-axis.

The Douglas- Peucker algorithm ran successfully on each horizon line ten times at ten different levels of tolerance. Using these simplified lines, it was possible to count the number of times each vertex was chosen as visually significant. The result of this was a dataset of points with a score of visual significance. The algorithm selected points that were most visually unique to the shape of the line more times than points that were not. The set of points was then filtered to only the points that were local maxima on the horizon lines and these points were stored in a horizon peaks dataset.

One noticeable bias of the algorithm was towards points on the horizon that were very close to the observer. A peak may have a low elevation but if it was near to the observer it would still have a much larger elevation angle than other peaks on the horizon. In terms of the algorithm, this was the desired output of the analysis. However, in the real world, some of the peaks that were chosen as visually significant might be relatively small hill that are only large because they are so close to the observer. These are not the types of peaks the client is interested in as visually significant.

The second goal of the project was to design a geodatabase to support the analysis. A geodatabase was built and implemented according to the logical design model using the ArcGIS version 9.3 file geodatabase format. The source data was loaded into the database. The geoprocessing tools were able to retrieve data from the geodatabase and store the results of their calculations in the geodatabase. The "SiteID" attribute was an 
essential part of the geodatabase schema. This field related they key datasets (Hopi villages, Horizons, and Horizon Peaks) to one another. The geodatabase had enough storage space to keep all the data and was reliably stored on a file server.

The geoprocessing tools produced by the Hopi Horizon Analysis were able to automate the entire calculation of visual significance for the dataset. The final Hopi Horizon Analysis toolset contained six custom scripted geoprocessing tools and one built in ArcGIS tools. These tools could be combined in one model to complete the entire analysis automatically.

Mapping the visual significance over time involved creating time snapshot maps of the aggregated visual significance of peaks in 50 year intervals. To do this, it was necessary to use the set of standard USGS peaks provided by the client and find the nearest horizon peak to each USGS peak. Each horizon peak that was in 400 meters of a USGS peak would contribute its visual significance score to the USGS peak's total visual significance score for each 50 year intervals. This method was only one possible way of representing the visual significance of horizon peaks. 


\section{Chapter 7 - Conclusions and Future Work}

The result of the Hopi Horizon Analysis project was a set of customized geoprocessing tools that allow the client to quantify the visual significance of peaks along a horizon relative to the location of the observer. The results of the visual significance analysis are intended to support the client's exploratory spatial data analysis of the horizons as seen by Hopi Native Americans as they migrated to different village locations in the Southwest from $1100-1700$ c.e. The final deliverables of the project are a geodatabase that stores and organizes the project data, a set of geoprocessing tools that automate the calculations for the entire dataset, and a set of maps that provide a visualization of the change in visual significance of horizon peaks over time.

This project was only the beginning stages of developing tools to measure the visual significance of horizon peaks. The next phase of this project will involve running the horizon analysis on a larger dataset of Hopi villages and horizons. A dataset with larger results should reveal more drastic differences in the change in total visual significance of horizon peaks. The project did not involve any field work to tests how the method for assessing how the method for quantifying visual significance of horizon peaks corresponds to the actual appearance of the horizons in the real world. Future work could involve going into the field and photographing horizon lines and then comparing these photographs of the appearance of the horizon lines to the visual significance ranks of the analysis results.

The original Hopi village dataset that the client provided had data representing the number of rooms that were occupied in each village for ever fifty year interval. The scope of this project did not take into consideration the size of each Hopi village. Future work could involve weighting the total visual significance of mountain peaks by the number of occupants in each village that could observe it.

When aggregating the visual significance scores of horizon peaks to the USGS Peaks dataset, any visually significant horizon peaks that were not within 400 meters of a USGS were not counted. In the future, the client could locate horizon peaks that did not aggregate to USGS peaks, create a new point dataset for these peaks, and combine this new dataset with the USGS peaks. The client could then aggregate the horizon peaks visual significance scores to the combined dataset using the Aggregate tool in the Horizon Analysis toolbox. The process for detecting where new peaks should be added could involve looking through the entire Horizon Peaks dataset and manually digitizing new points. Alternatively, a specialized cluster analysis could be developed to detect where clusters of horizon peaks that are not near USGS peaks occur.

A new version of the ArcGIS Desktop software was released before the Hopi Horizon Analysis was completed. Future work will require updating the geoprocessing tools and geodatabase so that they are compatible with the latest ArcGIS Desktop version 10 software, as well as future releases. Also, ArcGIS 10 has new functionality for visualizing temporal aspects of geographic data that could be applied to the challenge of mapping the change in visual significance of horizon peaks over time.

During the implementation of this project, Dr. Bernardini had also been working with Nathan Strout (Strout, 2011) of The Redlands Institute with other applications for visualizing peaks. One application currently in development is a tool that Dr. Bernardini 
can use in ArcMap that will allow him to create more accurate horizon line data locally, instead of depending on the "Hey, What's That?" web service. 


\section{Works Cited}

Bodum, L. (2004). Modelling Virtual Environments for Geovisualization: A Focus on Representation. In Dykes, J., MacEachren, A.M., \& Kraak, M.-J. (Eds.), Exploring Geovisualization (pp 389-401). Retrieved from: http://www.plan.aau.dk/ lbo/SIM/Geoviz_06/19-bodum.pdf

Christopherson, G.L. (2003, July). Using ARC/GRID to Calculate Topographic Prominence in an Archaeological Landscape. Paper presented at ESRI International User Conference, San Diego, CA. Retrieved from http://proceedings.esri.com/library/userconf/proc03/p0530.pdf.

Douglas, D.H. \& Peucker, T.K. (1973). Algorithms for the reduction of the number of points required to represent a digitized line or its caricature. Cartographica: The International Journal for Geographic Information and Geovisualization, 10(2), 112-122. doi:10.3138/FM57-6770-U75U-7727

Earl, E. \& Metzler, D. (2010). A new topographic functional. Retrieved from http://www.peaklist.org/spire/theory/paper.pdf

Esri, Simplifying Lines and Smoothing Features. (2009). ArcGIS Desktop 9.3 Help. Retrived from http://webhelp.esri.com/arcgiSDEsktop/9.3/index.cfm?TopicName=Simplifying and_smoothing features

Germino, M. J., Reiners, W. A., Blasko, B. J., McLeod, D., \& Bastian, C. T. (2001). Estimating visual properties of Rocky Mountain landscapes using GIS. Landscape and Urban Planning, 53(1-4), 71-83. doi:10.1016/S0169-2046(00)00141-9

Kosowsky, M. (2010, January). HeyWhatsThat Technical Frequently Asked Questions. Retrieved from http://www.heywhatsthat.com/techfaq.html

Kraak, M. -J. (2002). Geovisualization illustrated. ISPRS Journal of Photogrammetry and Remote Sensing, 57(5-6), 390-399. Doi: 10.1016/S0924-2716(02)00167-3

Llobera, M., (2003). Extending GIS-based visual analysis: the concept of visualscapes. International Journal of Geographical Information Sciences, 17(1), 25-48. Doi:10.1080/713811741

Maizlish, A. (2003). Prominence and Orometrics: A Study of the Measurement of Mountains. Retrieved from http://www.peaklist.org/theory/theory.html

Podobnikar, T. (2009, June). Method for Determination of the Mountain Peaks. Paper presented at the $12^{\text {th }}$ AGILE International Conference on Geographic Information Science, Hannover, Germany. Abstract retrieved from http://plone.itc.nl/agile_old/Conference/2009-hannover/pdfs/13.pdf

Saalfield, A. (1999). Topologically Consistent Line Simplification with the DouglasPeucker Algorithm. Cartography and Geographic Information Science, 26(1), 718.

Sinha, G. (2008). Delineation, characterization, and classification of topographic eminences. Dissertation Abstracts International: Section B. Sciences and Engineering, 68(12), 3291573.

Strout, N. (2011, February 11). Personal communication.

Visvalingam, M., \& Williamson, P. J. (1995). Simplification and Generalization of Large Scale Data for Roads: A Comparison of Two Filtering Algorithms. Cartography 
and Geographic Information Science, 22(4), 264-275.

doi:10.1559/152304095782540249

Worboys, M., \& Duckham, M. (2004). GIS: A Computing Perspective. Boca Raton, FL: CRC Press. 
